\title{
Construções consistentes de espaços de Banach $C(K)$ com poucos operadores
}

\author{
Rogério Augusto dos Santos Fajardo
}

TESE APRESENTADA

$\mathrm{AO}$

Instituto de MATemÁtica e EstatísticA

DA

UniversidAde DE SÃo PAUlo

PARA

OBTENÇÃO DO GRAU

$\mathrm{DE}$

Doutor EM CIÊNCIAS

Área de Concentração: Matemática

Orientador: Prof. Dr. Eloi Medina Galego

Co-orientador: Prof. Dr. Piotr Koszmider

Durante a elaboração deste trabalho, o autor recebeu apoio financeiro da FAPESP (processo 04/03508-6).

São Paulo, setembro de 2007 


\section{Construções consistentes de espaços de Banach $C(K)$ com poucos operadores}

Este exemplar corresponde à redação final da tese de doutorado devidamente corrigida e defendida por Rogério Fajardo

e aprovada pela comissão julgadora.

São Paulo, novembro de 2007.

Banca examinadora:

- Prof. Dr. Elói Medina Galego - IME-USP

- Profa. Dr. Ricardo Bianconi - IME-USP

- Prof. Dr. Jorge Túlio Mujica Ascui - UNICAMP

- Prof. Dr. Mário Carvalho de Matos - UNICAMP

- Prof. Dr. Valentin Ferenczi - PARIS VI 
À minha esposa, Joice, com toda estima, amor e carinho, pelo novo brilho que trouxe à minha vida, À memória de meu pai, Clóvis, que me introduziu o prazer pela matemática, e o valor da cidadania. 


\section{Agradecimentos}

Agradeço a minha mãe, sogros e toda a minha família, pelo carinho e atenção dispensados.

Agradeço a todos os professores do IME, especialmente aos meus orientadores.

Agradeço a todos os colegas do IME que conheci durante todos esses anos.

Agradeço à FAPESP pelo apoio financeiro.

Agradeço, com especialidade, aos meus professores do ginásio: Roberto, Rúbia e Hélio, do Colégio São Vicente de Paula. 


\section{Resumo}

Neste trabalho aplicamos técnicas de combinatória infinitária e forcing na teoria dos espaços de Banach, investigando propriedades dos espaços de Banach da forma $C(K)$ com poucos operadores, no sentido de que todo operador em $C(K)$ são da forma $g I+S$, onde $I$ é o operador identidade, $g \in C(K)$ e $S$ é fracamente compacto. Enfatizamos as construções onde $K$ é conexo, o que implica que $C(K)$ é indecomponível. Assumindo $\diamond$, um axioma combinatório mais forte que a Hipótese do Contínuo, construímos um espaço de Banach $C(K)$ tal que $C(L)$ tem poucos operadores, para todo $L \subseteq K$ fechado. Sob a Hipótese do Contínuo construímos um espaço $C(K)$ indecomponível com poucos operadores tal que $K$ contém $\beta \mathbb{N}$ homeomorficamente. Em ZFC construímos um espaço $C(K)$ com poucos operadores em um sentido estritamente

mais fraco. Também mostramos a existência de pelo menos contínuo espaços de Banach $C(K)$ indecomponíveis dois a dois essencialmente incomparáveis. Usando forcing provamos que existe consistentemente um espaço de Banach $C(K)$ de densidade menor que contínuo com poucos operadores e um $C(K)$ indecomponível de densidade menor que contínuo.

\section{Abstract}

In this work we apply techniques of infinitary combinatorics and forcing in Banach spaces theory, investigating the compact topological spaces $K$ such that the Banach space $C(K)$ has few operators, in the sense that all operators on $C(K)$ have the form $g I+S$, where $I$ is the identity operator, $g \in C(K)$ and $S$ is weakly compact. We emphasize the constructions where $K$ is connected, which implies that $C(K)$ is indecomposable. Assuming $\diamond$, a combinatoric axiom stronger than the continuum hypothesis, we construct a Banach space $C(K)$ where $C(L)$ has few operators, for every closed $L \subseteq K$. Under continuum hypothesis we construct an indecomposable $C(K)$ with few operators such that $K$ contains $\beta \mathbb{N}$ homeomorphically. In ZFC we construct a space $C(K)$ with few operators in a strictly weaker sense. We also show the existence of at least continuum essentially incomparable indecomposable Banach spaces $C(K)$. Using forcing, we prove that there exists consistently a Banach space $C(K)$ of density smaller than continuum having few operators and an indecomposable $C(K)$ of density smaller than continuum. 


\section{Sumário}

Notação $\quad$ xi

$\begin{array}{ll}\text { Introdução } & 1\end{array}$

1 Resultados preliminares $\quad 7$

1.1 Espaço de Banach $C(K)$ e o dual $M(K) \ldots \ldots \ldots$

1.2 Multiplicadores fracos . . . . . . . . . . . . . 10

1.3 Extensões por funções contínuas . . . . . . . . . . . . . . . 12

1.4 Axioma $\diamond \ldots \ldots \ldots \ldots \ldots$

2 Quocientes de espaços indecomponíveis da forma $C(K) \quad \mathbf{2 5}$

2.1 Um espaço $C(K)$ com muitos quocientes indecomponíveis . . 26

2.2 Um espaço $C(K)$ indecomponível tal que $K$ contém $\beta \mathbb{N} \ldots .37$

3 Outras construções de $C(K)$ com poucos operadores 41

3.1 Espaços de Banach $C(K)$ com quase poucos operadores . . . . 41

3.2 Construção de $2^{\omega}$ espaços $C(K)$ indecomponíveis essencialmente incomparáveis . . . . . . . . . . . . . . . . . . 43

4 Um espaço $C(K)$ de densidade $\omega_{1}<2^{\omega}$ com poucos operadores $\quad 49$

4.1 Convergência fraca em $M(K) \ldots \ldots \ldots \ldots$

4.2 Construção de um forcing . . . . . . . . . . . . . . . . . 53

4.3 Construção consistente de um espaço $C(K)$ de densidade menor que contínuo com poucos operadores . . . . . . . 56

5 Um espaço $C(K)$ indecomponível de densidade $\omega_{1}<2^{\omega} \quad \mathbf{6 7}$

5.1 Construção do forcing . . . . . . . . . . . . . . . 68

5.2 Iteração do forcing . . . . . . . . . . . . . . 76 
$\begin{array}{lr}\text { A Representação de Stone } & 87\end{array}$

A.1 Álgebras de Boole . . . . . . . . . . . . . . . . . . . 87

A.2 Teorema da Representação de Stone . . . . . . . . . . . . . . . 89

$\begin{array}{ll}\text { B Forcing } & 91\end{array}$

B.1 Modelos transitivos enumeráveis . . . . . . . . . . . . . . 91

B.2 Ordens parciais e filtros genéricos . . . . . . . . . . . . 93

B.3 Extensões genéricas . . . . . . . . . . . . . . . . . . . 95

B.4 Relação de forcing . . . . . . . . . . . . . . . . . . . . . 966

B.5 Absolutividade e preservação de cardinais . . . . . . . . . . . . 97

B.6 Forcing iterado . . . . . . . . . . . . . . . . . . . . . . 99

$\begin{array}{ll}\text { Bibliografia } & 101\end{array}$

$\begin{array}{ll}\text { Índice Remissivo } & 105\end{array}$ 


\title{
Notação
}

\author{
$\mathbb{R} \quad$ conjunto dos números reais \\ $\mathbb{Q} \quad$ conjunto dos números racionais \\ $\omega \quad$ conjunto dos naturais e o primeiro ordinal infinito enumerável \\ $\omega_{1} \quad$ primeiro ordinal não-enumerável \\ $\mathcal{P}(X) \quad$ conjunto das partes de $X$ \\ $|X| \quad$ cardinalidade de $X$ \\ $2^{\omega} \quad$ cardinal do contínuo, isto é, $|\mathcal{P}(\omega)|$ \\ $\mathrm{CH} \quad$ Hipótese do Contínuo, isto é, $\omega_{1}=2^{\omega}$ \\ $C(K)$ espaço de Banach das funções contínuas de $K$ em $\mathbb{R}$ com a norma supremo \\ $M(K)$ espaço de Banach das medidas de Radon em $K$ com a norma da variação \\ $\|x\| \quad$ norma de $x$ \\ $X^{*} \quad$ espaço dual do espaço de Banach $X$ \\ $T^{*} \quad$ operador adjunto do operador $T$ \\ $B_{X^{*}} \quad$ bola unitária de $X^{*}$ com a topologia fraca* \\ $l_{\infty} \quad$ espaço de Banach das seqüências limitadas em $\mathbb{R}$ com a norma supremo \\ $c_{0} \quad$ espaço de Banach das seqüências reais convergentes a 0, com a norma supremo \\ $X \oplus Y \quad$ soma direta de $X$ e $Y$ \\ $\beta \mathbb{N} \quad$ compactificado de Stone-Čech dos naturais \\ $\operatorname{dom}(f)$ domíno da função $f$ \\ $\operatorname{supp}(f)$ suporte da função $f$, isto é, $\overline{\{x \in \operatorname{dom}(f): f(x) \neq 0\}}$ \\ $G r(f)$ gráfico da função $f$, isto é, $\{(x, f(x)): x \in \operatorname{dom}(f)\}$ \\ $\operatorname{supp}(p)$ suporte de uma condição $p$ de um forcing iterado \\ ZFC sistema de axiomas de Zermelo-Frankel com Axioma da Escolha \\ MA axioma de Martin
}




\section{Introdução}

Na teoria dos espaços de Banach diversas perguntas foram feitas sobre quando um subespaço fechado $Y$ de um espaço de Banach $X$ é complementado em $X$, isto é, se existe $Z$ subespaço fechado de $Y$ tal que $X=Y \oplus Z$. Durante muitos anos ficou em aberto se para todo espaço de Banach $X$, de dimensão infinita, existem subespaços fechados $Y$ e $Z$, de dimensões infinitas, tais que $X=Y \oplus Z$. Quando isso ocorre, dizemos que $X$ é decomponível.

Em 1993 Gowers e Maurey construíram, em [GM1], o primeiro exemplo de um espaço de Banach indecomponível. Mais do que isso: o exemplo construído por eles é hereditariamente indecomponível, isto é, todos os seus subespaços fechados são indecomponíveis.

Uma vez que decomposições de espaços de Banach são dadas por operadores projeções, espaços indecomponíveis estão relacionados com espaços com poucos operadores. No espaço construído por Gowers e Maurey, todo operador é da forma $c I+S$, onde $I$ é o operador identidade, $c \in \mathbb{R}$ e $S$ é um

operador estritamente singular, isto é, nenhuma restrição a um subespaço de dimensão infinita é um isomorfismo sobre a imagem.

Diversos outros resultados sobre espaços de Banach que possuem poucos operadores têm sido publicados. Em 1978, assumindo o axioma $\diamond$ (veja Seção 1.4), Shelah mostra que existe um espaço de Banach de densidade $\omega_{1}$ cujos operadores são da forma $c I+S$, onde $c \in \mathbb{R}$ e $S$ tem imagem separável. Em 1988 Shelah e Steprans mostram o mesmo resultado sem assumir nenhum axioma adicional de teoria dos conjuntos.

Em [Ko2] encontramos o primeiro exemplo de um espaço de Banach indecomponível da forma $C(K)$, o espaço de Banach das funções reais contínuas sobre um compacto $K$ munido da norma do supremo. Nesse artigo há duas construções, na primeira $K$ é 0 -dimensional e na segunda é conexo, de espaços de Banach $C(K)$ em que todos os operadores são multiplicadores fracos (Definição 1.7). Como conseqüência, $C(K)$ não é isomorfo aos seus hiperplanos, 
nem a qualquer subespaço ou quociente próprio. Na construção conexa obtemos $C(K)$ indecomponível. Koszmider prova que, se $K \backslash F$ é conexo, para todo $F$ finito, e todo operador em $C(K)$ é multiplicador fraco, então $C(K)$ é indecomponível.

Assumindo a Hipótese do Contínuo (CH), Koszmider mostra que podemos obter um compacto $K$ tal que todo operador em $C(K)$ é da forma $g I+S$, para $g \in C(K)$ e $S$ fracamente compacto. Isso implica, quando $K$ é conexo, que $C(K)$ é indecomponível. Usando Representação de Wallman para reticulados conexos, que generaliza a representação de Stone para obter espaços conexos, em [Pl] Plebanek construiu um espaço de Banach $C(K)$ indecomponível com poucos operadores, no sentido como acima, sem assumir nenhum axioma adicional.

Como todo espaço de Banach de dimensão infinita da forma $C(K)$ contém $c_{0}$ como subespaço, $C(K)$ não pode ser hereditariamente indecomponível, como o espaço construído por Gowers e Maurey. O espaço construído em [Ko2] é um exemplo natural de um espaço de Banach indecomponível mas não hereditariamente indecomponível.

No contexto de $C(K)$, um operador é fracamente compacto se, e somente se, é estritamente singular (vide [Pe2]). Está verificado em [Ko2] que obter um espaço da forma $C(K)$ em que todo operador é da forma $c I+S$ onde $c \in \mathbb{R}$ e $S$ é fracamente compacto (equivalentemente, $S$ é estritamente singular), como o espaço obtido em [GM1], é impossível. Igualmente mostra-se que todo espaço $C(K)$ tem operador que não é da forma $g I+C$, para $g \in C(K)$ e $C$ um operador compacto. Assim, um espaço de Banach $C(K)$ onde todo operador é da forma $g I+S$, onde $g \in C(K)$ e $S$ é fracamente compacto, é o melhor que podemos esperar no sentido de poucos operadores em $C(K)$. A partir de agora, salvo menção contrária, essa será a definição de espaços $C(K)$ com poucos operadores.

Em 1999 Ferenczi, em [Fer], mostrou que o espaço construído por Gowers e Maurey, além de hereditariamente indecomponível, também é quociente hereditariamente indecomponível, isto é, todos seus quocientes são hereditariamente indecomponíveis. Nesse mesmo artigo, Ferenczi constrói um espaço hereditariamente indecomponível, mas não quociente hereditariamente indecomponível.

Como em [LM] mostra-se que espaços da forma $C(K)$ possuem $c_{0}$ complementado ou $l_{2}$ como quociente, espaços de Banach $C(K)$ também não podem ser quociente hereditariamente indecomponível, nem é possível que todos quocientes tenham poucos operadores. Porém, no Capítulo 2 cons- 
truímos $C(K)$ com muitos quocientes indecomponíveis. Assumindo $\diamond$, mostramos que existe um espaço de Banach $C(K)$ indecomponível tal que $C(L)$ tem poucos operadores, para todo $L \subseteq K$ fechado (Teorema 2.2), respondendo a uma pergunta deixada no final de [Ko2]. Em particular, mostramos que $C(K)$ como no Teorema 2.2 tem pelo menos contínuo quocientes indecomponíveis da forma $C(L)$ (Corolário 2.3), pois, se $L$ é um subespaço fechado de $K$, então $C(L)$ é um quociente de $C(K)$, induzido pelo operador $T: C(K) \longrightarrow C(K)$ dado por $T(f)=\left.f\right|_{L}$.

Uma pergunta que poderia surgir é se qualquer espaço indecomponível $C(K)$ construído por sucessivas extensões fortes, como o construído em [Ko2], já tem a propriedade acima. Assumindo $\mathrm{CH}$, obtemos um espaço $C(K)$ indecomponível tal que $\beta \mathbb{N} \subseteq K$ (Teorema 2.5), e $C(\beta \mathbb{N})$ é isométrico a $l_{\infty}$, que tem muitos operadores ${ }^{1}$. Porém, para $C(K)$ conter $l_{\infty}$ como qociente é suficiente, mas não necessário, que $K$ contenha $\beta \mathbb{N}$. Talagrand mostra, em [Ta], que $C(K)$ contém $l_{\infty}$ como quociente se, e somente se, $B_{C(K)^{*}}$ contém um subespaço homeomorfo a $\beta \mathbb{N}$. No caso do espaço construído no Teorema 2.2 não sabemos se ele contém $l_{\infty}$ como quociente.

Em 1975 Fedorchuk, em [Fed], mostrou, assumindo $\mathrm{CH}$, que existe um espaço topológico compacto $K$ que não contém seqüência convergente nem contém um subespaço isomorfo a $\beta \mathbb{N}$, respondendo, consistentemente, a uma pergunta dos anos 50 feita por Efimov. Um compacto $K$ como do Teorema 2.2 também responde negativamente ao problema de Efimov, mas assumimos $\diamond$, que é mais forte que $\mathrm{CH}$ (Lema 1.26). A consistência da negação do problema de Efimov permanece em aberto.

Na Seção 3.1, Teorema 3.2, mostramos uma construção de um compacto 0-dimensional $K$ tal que todo operador em $C(K)$ é multiplicador fraco mas nem todo é da forma $g I+S$. Essa construção foi obtida independentemente por Iryna Schlackow (vide [Schl]). O exemplo construído é o mesmo, apesar da demonstração ser diferente. Em [Schl] também mostra-se que a propriedade que todo operador em $C(K)$ é multiplicador fraco é invariante por isomorfismos de espaços de Banach, mas a propriedade de que todo operador em $C(K)$ é da forma $g I+S$, para $g \in C(K)$ e $S$ fracamente compacto, não é invariante por isomorfismos.

Podemos perguntar se um espaço indecomponível não é único, a menos

\footnotetext{
${ }^{1} \mathrm{O}$ espaço topológico $\beta \mathbb{N}$ está definido no Apêndice A, Definição A.12. A isometria entre $C(\beta \mathbb{N})$ e $l_{\infty}$ segue do Teorema A.13. De resultados de [Ko2] e [Sc], que mencionaremos nesta Introdução, e do fato que $l_{\infty}=c_{0} \oplus l_{\infty}$, seguem que $l_{\infty}$ não tem poucos operadores, no sentido por nós adotado.
} 
de isomorfismos, e, se não é, quanto deles existem, não-isomorfos. Em [Ga], Gasparis construiu uma família de cardinalidade $2^{\omega}$ de espaços de Banach indecomponíveis separáveis (como os espaços de [GM1]) que são dois a dois totalmente incomparáveis, isto é, nenhum subespaço de dimensão infinita de um espaço é isomorfo a um subespaço de outro. Como os espaços são separáveis, $2^{\omega}$ é o maior cardinal possível para essa família.

Como $C(K)$ de dimensão infinita contém $c_{0}$ como subespaço, dois espaços de Banach da forma $C(K)$ não podem ser totalmente incomparáveis. Um outro conceito de incomparabilidade, desenvolvida por Aiena e Gonzalez (vide $[\mathrm{AG}]$ ), é o de espaços de Banach essencialmente incomparáveis (Definição 3.3). O Teorema 3.5 mostra a existência de uma família de $2^{\omega}$ espaços de Banach indecomponíveis da forma $C(K)$ essencialmente incomparáveis.

Chamamos de densidade de um espaço de Banach $X$ o menor cardinal $\lambda$ para o qual existe um denso em $X$ de cardinalidade $\lambda$. Por exemplo, dizer que $X$ tem densidade enumerável significa que $X$ é separável. Podemos estudar os possíveis valores para a densidade de espaços $C(K)$ indecomponíveis, ou com poucos operadores.

Um espaço de Banach é de Grothendieck (ou, possui a propriedade de Grothendieck) se a convergência fraca e a convergência fraca* coincidem no espaço dual. Schachermayer mostra, em [Sc], que um espaço de Banach da forma $C(K)$ é de Grothendieck se, e somente se, não contém $c_{0}$ complementado. Em [Ko2] mostra-se, usando o resultado de Schachermayer, que, se $C(K)$ é indecomponível, tem poucos operadores, ou todos os operadores nele são multiplicadores fracos, então $C(K)$ é de Grothendieck.

O espaço de Banach indecomponível construído em [GM1] é separável. Espaços de Banach da forma $C(K)$ indecomponíveis, ou com poucos operadores (mesmo no sentido de todo operador em $C(K)$ ser multiplicador fraco), não podem ser separáveis, pois todo espaço $C(K)$ separável contém uma cópia complementada de $c_{0}$ e, portanto, não é de Grothendieck. Todos os espaços de Banach da forma $C(K)$ construídos em [Ko2], [Pl] e nos Capítulos 2 e 3 têm densidade $2^{\omega}$.

Por um resultado de Fremlin, em [Fr], axioma de Martin (vide [Ku]) implica que, se $K$ tem peso menor que $2^{\omega}$, então $C(K)$ tem uma cópia complementada de $c_{0}$ e, portanto, não é de Grothendieck e, em particular, não pode ter poucos operadores (veja introdução do Capítulo 4 para mais detalhes).

Em $[\mathrm{Br}]$ Brech mostra o primeiro exemplo consistente de um espaço $C(K)$ de densidade menor que contínuo que possui a propriedade de Grothendieck. Mas, como mostramos na introdução do Capítulo 4, o espaço $C(K)$ cons- 
truído por [Br] contém operadores que não são multiplicadores fracos.

No Capítulo 4, Teorema 4.14, mostramos consistentemente, usando forcing iterado, a existência de um espaço de Banach $C(K)$ de densidade menor que $2^{\omega}$ com poucos operadores. Adaptando essa construção para $K$ conexo, no Capítulo 5 mostramos a consistência da existência de um espaço $C(K)$ indecomponível de densidade menor que contínuo. Por $[\mathrm{Fr}]$ sabemos que ambos resultados, assim como o de $[\mathrm{Br}]$, são independentes de $\mathrm{ZFC}+\neg \mathrm{CH}$. Como $C(K)$ separável não possui a propriedade de Grothendieck, $\mathrm{CH}$ implica a negação desses resultados.

Esta tese está dividida da seguinte maneira: No Capítulo 1 apresentamos alguns resultados clássicos sobre a teoria dos espaços de Banach, definições e resultados sobre multiplicadores fracos e extensões por funções contínuas, a maioria deles extraídos de [Ko2], e uma seção sobre o axioma $\diamond$. No Capítulo 2 mostramos, assumindo $\diamond$, a existência de um compacto conexo $K$ tal que $C(L)$ tem poucos operadores, para todo $L \subseteq K$ (Teorema 2.2). Assumindo $\mathrm{CH}$ também construímos um $C(K)$ com poucos operadores, com $K$ conexo, tal que $\beta \mathbb{N} \subseteq K$ (Teorema 2.5). No Capítulo 3 construímos um $C(K)$ no qual todo operador é multiplicador fraco mas nem todo é da forma $g I+S$, para $g \in C(K)$ e $S$ fracamente compacto (Teorema 3.2), e construímos $2^{\omega}$ espaços $C(K)$ indecomponíveis dois a dois essencialmente incomparáveis (Teorema 3.5). No Capítulo 4 encontramos a construção (consistente) de um espaço $C(K)$ de densidade menor que contínuo com poucos operadores (Teorema 4.14). No Capítulo 5, adaptando a construção do Capítulo 4 para o caso $K$ conexo, mostramos consistentemente a existência de um espaço de Banach $C(K)$ indecomponível de densidade menor que contínuo. Os Apêndices A e B fornecem breves descrições sobre a representação de Stone e a técnica do forcing, respectivamente, essenciais para a compreensão dos Capítulos 4 e 5 . 


\section{Capítulo 1}

\section{Resultados preliminares}

Neste capítulo, apresentaremos alguns resultados que serão utilizados no decorrer da tese. A Seção 1.1 apresenta alguns resultados clássicos da teoria dos espaços de Banach. As Seções 1.2 e 1.3 apresentam definições e resultados que constam em [Ko2], sobre multiplicadores fracos e extensões de compactos conexos por funções contínuas. Apresentamos, nessas seções, algumas variações dos lemas de [Ko2] que serão necessárias para os Capítulos 2 e 3. A Seção 1.4 trata do axioma $\diamond$, que será usado no Capítulo 2.

\subsection{Espaço de Banach $C(K)$ e o dual $M(K)$}

Os borelianos sobre um compacto $K$ são os elementos da $\sigma$-álgebra gerada pelos abertos, isto é, o menor subconjunto de $\mathcal{P}(K)$ que contém os abertos de $K$ e é fechado por uniões enumeráveis e complementos. Seja $\mathcal{B}(K)$ o conjunto dos borelianos de $K$. Uma medida boreliana em $K$ é uma função $\sigma$-aditiva $\mu$ de $\mathcal{B}(K)$ em $\mathbb{R}$. Isto é, se $E=\bigcup_{n \in \omega} E_{n}$, onde $\left(E_{n}\right)_{n \in \omega}$ são borelianos em $K$ dois a dois disjuntos, então

$$
\mu(E)=\Sigma_{n \in \omega} \mu\left(E_{n}\right) .
$$

Uma medida boreliana sobre $K$ é positiva se $\mu(E) \geq 0$, para todo $E \subseteq K$ boreliano. Dada uma medida boreliana $\mu$, definimos $|\mu|$ por

$|\mu|(E)=\sup \left\{\Sigma_{1 \leq i \leq n}\left|\mu\left(E_{n}\right)\right|: n \in \omega, E_{i} \in \mathcal{B} E_{i} \subseteq E, E_{i} \cap E_{j}=\emptyset\right.$, se $\left.i \neq j\right\}$.

Temos que $|\mu|$ está bem definida como função de $\mathcal{B}(K)$ em $\mathbb{R}$ e é uma medida positiva (veja $[\mathrm{Ru}]$, Teoremas 6.2 e 6.4). Chamamos a medida $|\mu|$ de variação de $\mu$. 
Uma medida $\mu$ é dita regular se para todo boreliano $E$ e todo $\varepsilon>0$ existem um fechado $F \subseteq E$ e um aberto $V \supseteq E$ tais que $|\mu|(V \backslash F)<\varepsilon$. Uma medida boreliana regular também é chamada de medida de Radon. Segue imediatamente da definição de regularidade que, se $\mu$ é regular, então $|\mu|$ também é regular.

A partir de agora convencionamos que o termo medida será usado no sentido de medida de Radon. Veremos, a seguir, que uma medida em um compacto $K$ é unicamente determinada pelos seus valores em uma base fechada por intersecções finitas.

Lema 1.1. Sejam $K$ um compacto e $B$ uma base de abertos de $K$ fechada por intersecções finitas. Se $\mu$ e $\nu$ são medidas sobre $K$ tais que $\left.\mu\right|_{B}=\left.\nu\right|_{B}$, então $\mu=\nu$.

Demonstração: Se $E_{1} \subseteq E_{2}$, para $E_{1}$ e $E_{2}$ borelianos em $K$, pela $\sigma$ aditividade de $\mu$ e $\nu$ temos $\mu\left(E_{2} \backslash E_{1}\right)=\mu\left(E_{2}\right)-\mu\left(E_{1}\right)$ e $\nu\left(E_{2} \backslash E_{1}\right)=$ $\nu\left(E_{2}\right)-\nu\left(E_{1}\right)$. Vejamos que, se $V$ é uma união finita de elementos de $B$, então $\mu(V)=\nu(V)$. Provaremos por indução em $n$ que, se $X \subseteq B$ e $|X|=n$, então $\mu(\cup X)=\nu(\cup X)$. O passo inicial $n=1$ temos pela hipótese. Suponha que $\mu$ e $\nu$ coincidem para todas uniões de no máximo $n$ elementos de $B$. Seja $V=\cup\left\{V_{i}: 1 \leq i \leq n+1\right\}$, onde $V_{i} \in B$. Temos

$$
V=\left(V_{n+1} \backslash \bigcup_{1 \leq i \leq n}\left(V_{n+1} \cap V_{i}\right)\right) \cup\left(\bigcup_{1 \leq i \leq n} V_{i}\right) .
$$

Como $B$ é fechado por intersecções finitas, $V_{n+1} \cap V_{i} \in B$. Logo, por $\left.\mu\right|_{B}=\left.\nu\right|_{B}$ e pela hipótese indutiva, tomando $V^{\prime}=\bigcup_{1 \leq i \leq n}\left(V_{n+1} \cap V_{i}\right)$ e $V^{\prime \prime}=\bigcup_{1 \leq i \leq n}\left(V_{i}\right)$ temos

$$
\mu(V)=\mu\left(V_{n+1}\right)-\mu\left(V^{\prime}\right)+\mu\left(V^{\prime \prime}\right)=\nu\left(V_{n+1}\right)-\nu\left(V^{\prime}\right)+\nu\left(V^{\prime \prime}\right)=\nu(V) .
$$

Seja $E \subseteq K$ boreliano. Para cada $n \in \omega, n>0$, usando regularidade de $\mu$ e $\nu$ tomamos fechados $F_{n}^{1}$ e $F_{n}^{2}$ contidos em $E$ e abertos $V_{n}^{1}$ e $V_{n}^{2}$ contendo $E$ tais que $|\mu|\left(V_{n}^{1} \backslash F_{n}^{1}\right)<\frac{1}{4 n}$ e $|\nu|\left(V_{n}^{2} \backslash F_{n}^{2}\right)<\frac{1}{4 n}$. Tomando $V_{n}=V_{n}^{1} \cap V_{n}^{2}$ e $F_{n}=F_{n}^{1} \cup F_{n}^{2}$ temos $F_{n} \subseteq E \subseteq V_{n},|\mu|\left(V_{n} \backslash F_{n}\right)<\frac{1}{4 n}$ e $|\nu|\left(V_{n} \backslash F_{n}\right)<\frac{1}{4 n}$.

Como $K$ é compacto e $F_{n}$ é fechado em $K$, temos que $F_{n}$ é compacto. Portanto existe $W_{n}$ união finita de abertos pertencentes a $B$ tal que $F_{n} \subseteq$ $W_{n} \subseteq V_{n}$. Temos

$$
\left|\mu(E)-\mu\left(W_{n}\right)\right| \leq\left|\mu(E)-\mu\left(F_{n}\right)\right|+\left|\mu\left(W_{n}\right)-\mu\left(F_{n}\right)\right|=
$$




$$
\left|\mu\left(E \backslash F_{n}\right)\right|+\left|\mu\left(W_{n} \backslash F_{n}\right)\right| \leq|\mu|\left(E \backslash F_{n}\right)+|\mu|\left(W_{n} \backslash F_{n}\right) \leq \frac{1}{2 n} .
$$

Analogamente, $\left|\nu(E)-\nu\left(W_{n}\right)\right|<\frac{1}{2 n}$. Como mostramos que $\mu\left(W_{n}\right)=\nu\left(W_{n}\right)$, concluímos que

$$
|\mu(E)-\nu(E)| \leq\left|\mu(E)-\mu\left(W_{n}\right)\right|+\left|\nu(E)-\nu\left(W_{n}\right)\right|<\frac{1}{n},
$$

para todo $n$. Portanto, $\mu(E)=\nu(E)$.

Seja $I$ um conjunto e seja $X=\prod_{i \in I} X_{i}$ o produto de espaços topológicos $X_{i}$. Definimos um aberto elementar de $X$ como um aberto da forma $\prod_{i \in I} V_{i}$, onde $V_{i}$ é um aberto básico (de uma base fixada) em $X_{i}$, para todo $i \in I$, e $V_{i}=X_{i}$ para todos, exceto finitos, $i \in I$. Se $Y \subseteq \prod_{i \in I} X_{i}$ é um subespaço chamamos de aberto elementar de $Y$ um aberto elementar de $\prod_{i \in I} X_{i}$ interceptado com $Y$. Segue da definição de topologia produto e de subespaço topológico que os abertos elementares formam uma base de abertos para $Y$, e é fácil ver que os abertos elementares de $Y$ formam uma base fechada por intersecções finitas.

No caso de $X_{i}=[0,1]$, consideraremos como abertos básicos os intervalos abertos em $[0,1]^{1}$ de extremos racionais.

Pelo Lema 1.1 podemos identificar uma medida de $[0,1]^{\kappa}$, para um cardinal $\kappa$, com uma função dos abertos básicos em $\mathbb{R}$. Se $K$ é o espaço de Stone de uma álgebra de Boole (veja Apêndice A) podemos identificar uma medida de $K$ como uma função $\sigma$-aditiva da álgebra em $\mathbb{R}$.

Uma função $f: K \longrightarrow \mathbb{R}$ é dita boreliana se $f^{-1}(V)$ é boreliano em $K$, para todo aberto $V \subseteq \mathbb{R}$.

Durante toda a tese, identificaremos o dual $C(K)^{*}$ de $C(K)$ com o espaço das medidas de Radon sobre $K$, que denotaremos por $M(K)$, com a norma dada por $\|\mu\|=|\mu|(K)$. Iremos trabalhar com as topologias fraca e fraca* de $M(K)$. Indicamos [Fa], [Se] ou [Di] para referências.

Os próximos dois teoremas encontram-se demonstrados em [Di].

Teorema 1.2 (Dieudonné-Grothendieck). ([Di], VII. 14) Um conjunto limitado $S \subseteq M(K)$ é fracamente relativamente compacto se, e somente se, para toda seqüência $\left(V_{n}\right)_{n \in \omega}$ de abertos dois a dois disjuntos de $K, \mu\left(V_{n}\right)$ converge

\footnotetext{
${ }^{1}$ Por intervalo aberto em $[0,1]$ queremos dizer que é aberto como subespaço de $[0,1]$. Isso inclui os intervalos da forma $[0, r),(r, 1]$, para $0<r<1$, e o próprio $[0,1]$.
} 
a 0 uniformemente em $S$, isto é,

$$
\sup _{\mu \in S}\left|\mu\left(V_{n}\right)\right| \stackrel{n \rightarrow \infty}{\longrightarrow} 0 .
$$

Teorema 1.3 (Rosenthal). ([Di], pag. 82) Seja $\left(\mu_{n}\right)_{n \in \omega}$ uma seqüência limitada em $M(K)$. Para todo $\varepsilon>0$ e toda seqüência $\left(A_{n}\right)_{n \in \omega}$ de subconjuntos borelianos de $K$ dois a dois disjuntos, existe uma seqüência estritamente crescente de inteiros $\left(k_{n}\right)_{n \in \omega}$ tal que

$$
\left|\mu_{k_{n}}\right|\left(\bigcup_{n \neq j}\left(A_{k_{j}}\right)\right)<\varepsilon
$$

para todo $n \in \omega$.

O Teorema 1.3 também é conhecido como Lema de Rosenthal.

Para o próximo teorema indicamos [Fa], Teorema 4.47.

Teorema 1.4 (Eberlein-Šmulian). ([Fa], 4.47) Um subconjunto $A$ de um espaço de Banach $X$ é fracamente relativamente compacto se, e somente se, toda seqüência em A admite uma subseqüência fracamente convergente.

Lema 1.5. ([DU], VI, Cor. 17) Sejam $K$ um espaço compacto e $X$ um espaço de Banach. Um operador $S: C(K) \longrightarrow X$ é fracamente compacto se, e somente se, para toda seqüência limitada e duas a duas disjunta $\left(f_{n}\right)_{n \in \omega}$ em $C(K)$, a seqüência $\left(\left\|S\left(f_{n}\right)\right\|\right)_{n \in \omega}$ converge a 0 .

Teorema 1.6 (Gantmacher). ([DS], VI 4.8) Um operador $T: X \longrightarrow Y$ é fracamente compacto se, e somente se, o operador adjunto $T^{*}: Y^{*} \longrightarrow X^{*} e ́$ fracamente compacto.

\subsection{Multiplicadores fracos}

A definição de multiplicadores fracos, também chamados de operadores centrípetos, aparece em [Ko2] para obter um espaço $C(K)$ indecomponível. Citaremos nesta seção os principais resultados referentes a multiplicadores fracos. Com exceção do Lema 1.12, todos os resultados desta seção encontram-se demonstrados em [Ko2]. 
Definição 1.7. ([Ko2], 2.1) Um operador $T: C(K) \longrightarrow C(K)$ é um multiplicador fraco se para toda seqüência limitada $\left(e_{n}: n \in \omega\right)$ de elementos dois a dois disjuntos de $C(K)$, e toda seqüência $\left(x_{n}: n \in \omega\right) \subseteq K$ tal que $e_{n}\left(x_{n}\right)=0$, temos

$$
\lim _{n \rightarrow \infty} T\left(e_{n}\right)\left(x_{n}\right)=0
$$

Lema 1.8. ([Ko2], 2.3) Seja $T: C(K) \longrightarrow C(K)$ um multiplicador fraco. Então $T$ é um isomorfismo sobre a imagem se, e somente se, $T$ é sobrejetor em $C(K)$.

Teorema 1.9. ([Ko2], 2.5) Suponha que todos os operadores em $C(K)$ são multiplicadores fracos e que $K \backslash F$ é conexo, para todo $F \subseteq K$ finito. Então $C(K)$ é um espaço de Banach indecomponível.

Lembramos que $Y \subseteq X$ é $C^{*}$-imerso em $X$ se, e somente se, toda função contínua e limitada de $Y$ em $\mathbb{R}$ se estende a uma função contínua e limitada de $X$ em $\mathbb{R}$.

Lema 1.10. ([Ko2], 2.8) Seja $K$ um espaço compacto $K$ tal que, para todos $U_{1}$ e $U_{2}$ abertos disjuntos, $\bar{U}_{1} \cap \bar{U}_{2}=\emptyset$ ou $\left|\bar{U}_{1} \cap \bar{U}_{2}\right| \geq 2$. Então para todo $x \in K$ o espaço $K \backslash\{x\}$ é $C^{*}$-imerso em $K$.

Teorema 1.11. ([Ko2], 2.7) São equivalentes para um espaço compacto $K$ :

a) Todo operador $T: C(K) \longrightarrow C(K)$ é da forma $g I+S$, onde $g \in C(K)$ e $S$ é fracamente compacto.

b) Todos os operadores em $C(K)$ são multiplicadores fracos e, para todo $x \in K$, o espaço $K \backslash\{x\}$ é $C^{*}$-imerso em $K$.

O lema seguinte é uma adaptação do Lema 2.5 de [Ko2].

Lema 1.12. Seja $K$ compacto e conexo tal que todos os operadores em $C(K)$ são da forma $g I+S$, onde $g \in C(K)$ e $S$ é um operador fraco. Então $C(K)$ é um espaço de Banach indecomponível. 
Demonstração: Seja $K$ como na hipótese do lema e sejam $X$ e $Y$ subespaços fechados de $C(K)$ tais que $C(K)=X \oplus Y$. Mostraremos que $X$ ou $Y$ tem dimensão finita.

Seja $P: C(K) \longrightarrow C(K)$ uma projeção tal que $\operatorname{Im}(P)=X$ e $\operatorname{Ker}(P)=$ $Y$. Pela hipótese existem $g \in C(K)$ e $S$ operador fracamente compacto tais que $P=g I+S$. Como $P^{2}=P$ temos $P^{2} I+S^{2}+g S+S g=g I+S$. Logo $S^{\prime}=\left(g^{2}-g\right) I$ é fracamente compacto e, portanto, estritamente singular (vide $[\mathrm{Pe} 2])$. Se $\left(g^{2}-g\right)(x) \neq 0$ para algum $x \in K$ achamos uma vizinhança aberta $V$ de $x$ tal que $\left|\left(g^{2}-g\right)(y)\right|>\varepsilon$, para algum $\varepsilon>0$ e todo $y \in \bar{V}$. Seja $Z$ o subespaço de $C(K)$ formado pelas funções que têm suporte em $V$. Como $K$ é conexo e, portanto, não tem pontos isolados, $Z$ tem dimensão infinita. Mas $\left.S^{\prime}\right|_{Z}$ é um isomorfismo sobre a imagem, pois $\left(g^{2}-g\right)^{-1}$ está bem definida e é contínua em $\bar{V}$, e determina uma inversa de $S^{\prime}$. Isso contradiz que $S^{\prime}$ é estritamente singular.

Logo $\left(g^{2}-g\right)(x)=0$, para todo $x \in K$. Portanto $g(x) \in\{0,1\}$, para todo $x \in K$. Como $K$ é conexo, $g \equiv 0$ ou $g \equiv 1$. Logo $P=S$ ou $P=I+S$, ou seja $P$ ou $I-P$ é fracamente compacto. No primeiro caso $\left.P\right|_{I m(P)}$ é um isomorfismo sobre a imagem (pois é a identidade), $\log \operatorname{Im}(P)$ tem dimensão finita. No segundo caso, $\left.(I-P)\right|_{\operatorname{Ker}(P)}$ é a identidade, e, portanto, $\operatorname{Ker}(P)$ tem dimensão finita, como queríamos.

\subsection{Extensões por funções contínuas}

Usando a representação de Stone (veja Apêndice A) identificamos espaços compactos 0-dimensionais (isto é, espaços com base de abertos-fechados) com álgebras de Boole. Através dessa dualidade podemos adicionar supremos de funções características de abertos-fechados dois a dois disjuntos adicionando o supremos na álgebra de abertos-fechados do espaço. Em [Ko2] encontramos uma forma de adicionar supremo em um compacto conexo, baseada no caso 0-dimensional e na representação de Stone. Iremos apresentar os resultados e definições da Seção 4 de [Ko2], e também algumas variações de resultados de [Ko2] que usaremos no decorrer da tese.

Definição 1.13. Se $K$ é um espaço compacto, e $\left(f_{n}\right)_{n \in \omega}$ é uma seqüência de funções contínuas duas a duas disjuntas de $K$ em $[0,1]$, definimos

- $D\left(\left(f_{n}\right)_{n \in \omega}\right)=\left\{x \in K: \exists U\right.$ vizinhança de $x$ tal que $U \cap \operatorname{supp}\left(f_{n}\right) \neq \emptyset$ para finitos $n \in \omega\}$; 
- $\Delta\left(\left(f_{n}\right)_{n \in \omega}\right)=K \backslash D\left(\left(f_{n}\right)_{n \in \omega}\right)$.

Lema 1.14. ([Ko2], 4.1) Seja $K$ um espaço compacto, e seja $\left(f_{n}\right)_{n \in \omega}$ uma seqüência de funções contínuas duas a duas disjuntas de $K$ em [0,1]. Então:

a) $D\left(\left(f_{n}\right)_{n \in \omega}\right)$ é um aberto denso em $K$;

b) $\Sigma_{n \in \omega} f_{n}$ é contínua em $D\left(\left(f_{n}\right)_{n \in \omega}\right)$.

Definição 1.15. ([Ko2], 4.2) Sejam $K$ um espaço compacto, $L \subseteq K \times[0,1]$ e $\left(f_{n}\right)_{n \in \omega}$ uma seqüência de funções contínuas duas a duas disjuntas de $K \mathrm{em}$ $[0,1]$. Dizemos que $L$ é uma extensão de $K$ por $\left(f_{n}\right)_{n \in \omega}$, e denotaremos por $K\left(\left(f_{n}\right)_{n \in \omega}\right)$, se $L$ é o fecho do gráfico de $\Sigma_{n \in \omega} f_{n} \mid D\left(\left(f_{n}\right)_{n \in \omega}\right)$. Dizemos que $L$ é uma extensão forte se, além disso, contém o gráfico de $\Sigma_{n \in \omega} f_{n}$.

O próximo lema é uma conseqüência imediata do Lema 1.14 e da definição de extensão.

Lema 1.16. Seja L uma extensão de $K$ por $\left(f_{n}\right)_{n \in \omega}$, uma seqüência duas a duas disjunta de funções contínuas de $K \mathrm{em}[0,1]$. Então

$$
\left\{x \in K:\left|\pi_{L, K}^{-1}(x)\right|>1\right\} \subseteq \Delta\left(\left(f_{n}\right)_{n \in \omega}\right)
$$

Dizemos que um subcojunto $M$ de um espaço topológico $K$ é raro se seu fecho tem interior vazio, isto é, se não existe um aberto $V$ não-vazio tal que $V \subseteq \bar{M}$

Lema 1.17. ([Ko2], 4.3) Sejam $K$ um espaço compacto e $\left(f_{n}\right)_{n \in N}$ uma seqüência de funções duas a duas disjuntas de $K$ em $[0,1]$ e seja $L=$ $K\left(\left(f_{n}\right)_{n \in \omega}\right)$. Considere $\pi$ a projeção de $L$ em $K$. Temos:

a) $S e M \subseteq K$ é raro em $K$ então $\pi^{-1}[M]$ é raro em $L$;

b) Existe $\sup \left\{f_{n} \circ \pi: n \in \omega\right\}$ em $C(L)$.

Lema 1.18. ([Ko2], 4.4) Seja $K$ um compacto e seja $\left(f_{n}\right)_{n \in \omega}$ uma seqüência de funções contínuas duas a duas disjuntas de $K$ em [0,1]. Se $K$ é conexo, então o gráfico de $\Sigma_{n \in \omega} f_{n}$ é conexo. Em particular, uma extensão forte de um compacto conexo também é conexa. 
Lema 1.19. ([Ko2], 4.5) Suponha que $K$ é compacto, de peso $\kappa<2^{\omega}$, e sejam $X_{1}, X_{2} \subseteq K$ subconjuntos disjuntos relativamente discretos em $K$ tais que $\bar{X}_{1} \cap \bar{X}_{2} \neq \emptyset$. Seja $\left(f_{n}\right)_{n \in \omega}$ uma seqüência duas a duas disjunta de funções contínuas de $K$ em $[0,1]$ e seja $\left(N_{\xi}: \xi<2^{\omega}\right)$ uma familia de subconjuntos infinitos de $\omega$ tal que $N_{\xi} \cap N_{\xi^{\prime}}$ é finito, para $\xi \neq \xi^{\prime}$. Então existe $A \subseteq 2^{\omega}$ de cardinalidade menor ou igual a $\kappa$ tal que, para todo $\xi \in 2^{\omega} \backslash A$ e todo $b \subseteq N_{\xi}$ infinito temos:

a) $K\left(\left(f_{n}\right)_{n \in b}\right)$ é uma extensão forte;

b) $\overline{\left\{\left(x,\left(\Sigma_{n \in b} f_{n}\right)(x)\right): x \in X_{1}\right\}} \cap \overline{\left\{\left(x,\left(\Sigma_{n \in b} f_{n}\right)(x)\right): x \in X_{2}\right\}} \neq \emptyset$, em $K\left(\left(f_{n}\right)_{n \in b}\right)$.

Para enunciar o próximo lema recordamos a definição de limite inverso de espaços topológicos. Seja $\Pi_{\alpha<\kappa} X_{\alpha}$ um produto de espaços topológicos, para $\kappa$ um ordinal limite. Sejam $Y_{\alpha}$ subespaços de $\Pi_{\beta<\alpha} X_{\beta}$ tais que $\pi_{\beta}\left[Y_{\alpha}\right]=Y_{\beta}$, para $\beta<\alpha$. Definimos o limite inverso de $\left(Y_{\alpha}\right)_{\alpha<\kappa}$ por

$$
\lim _{\leftarrow}\left(Y_{\alpha}\right)_{\alpha<\kappa}=\left\{\left(y_{\alpha}\right)_{\alpha<\kappa} \in \Pi_{\alpha<\kappa} X_{\alpha}: \forall \alpha<\kappa\left(\left(y_{\beta}\right)_{\beta<\alpha} \in Y_{\alpha}\right)\right\} .
$$

Limite inverso preserva compacidade (vide [Eng], 2.5.1).

Lema 1.20. ([Ko2], 4.6) Seja $\beta$ um ordinal e seja $\left(K_{\alpha}\right)_{\alpha \leq \beta}$ tal que $K_{2}=$ $[0,1]^{2}, K_{\alpha} \subseteq[0,1]^{\alpha}$ é compacto, $K_{\alpha}$ é o limite inverso de $\left(K_{\gamma}\right)_{\gamma<\alpha}$, se $\alpha$ é limite, e $K_{\alpha+1}$ é uma extensão forte de $K_{\alpha}$ por funções duas a duas disjuntas de $K_{\alpha}$ em [0,1]. Então

a) Se $f, f_{n} \in C\left(K_{\alpha}\right)$, para $n \in \omega$, e $\alpha \leq \beta$ são tais que

$$
f=\sup \left\{f_{n}: n \in \omega\right\}
$$

então

$$
f \circ \pi_{\beta, \alpha}=\sup \left\{f_{n} \circ \pi_{\beta, \alpha}: n \in \omega\right\}
$$

b) $K_{\beta} \backslash F$ é conexo, se $F \subseteq K_{\beta}$ é finito.

Os próximos três lemas são variações do Lema 1.19, que serão utilizados no Capítulo 2.

Lema 1.21. Seja K um espaço compacto de peso enumerável sem pontos isolados. Sejam $\left(\varepsilon_{n}\right)_{n \in \omega}$ uma seqüência de reais positivos, $\left(g_{n}\right)_{n \in \omega}$ uma seqüência de funções contínuas duas a duas disjuntas de $K$ em $[0,1],\left(\mu_{n}\right)_{n \in \omega}$ uma seqüência de medidas e $\left(x_{n}\right)_{n \in \omega}$ uma seqüência em $K$ tal que $g_{n}\left(x_{n}\right)=1$. Então existem funções contínuas $f_{n}: K \longrightarrow[0,1]$ tais que 
(a) Para todo $n \in \omega, \operatorname{supp}\left(f_{n}\right) \subseteq \operatorname{supp}\left(g_{n}\right), f_{n}\left(x_{n}\right)=1$ e $\int\left|f_{n}-g_{n}\right| d\left|\mu_{n}\right|<$ $\varepsilon_{n}$

(b) Se $\pi$ é a projeção de $K\left(\left(f_{n}\right)_{n \in \omega}\right)$ em $K$, para todo $x \in K \backslash D\left(\left(f_{n}\right)_{n \in \omega}\right)$ temos que $\pi^{-1}\{x\}=\{x\} \times[0,1]$.

Demonstração: Como $K$ é compacto e tem peso enumerável, $K$ é metrizável. Fixe $d$ uma métrica em $K$. Para cada $n \in \omega$ fixamos uma família de abertos $\left\{V_{n}^{i}: i \in I_{n}\right\}$, para $I_{n}$ finito, tal que cada $V_{n}^{i}$ tem diâmetro menor ou igual a $\frac{1}{n}$ (isto é, $\left.\sup \left\{d(x, y): x, y \in V_{n}^{i}\right\} \leq \frac{1}{n}\right)$ e $K=\bigcup_{i \in I_{n}} V_{n}^{i}$. Consideremos

$$
I_{n}^{\prime}=\left\{i \in I_{n}:\left\{j \in \omega: V_{n}^{i} \cap \operatorname{supp}\left(g_{j}\right) \neq \emptyset\right\} \text { é finito }\right\}
$$

Construiremos, por indução em $n$, conjuntos finitos, dois a dois disjuntos, $F_{n} \subseteq \omega$ e funções $\left\{f_{k}: k \in F_{n}\right\}$ de $K$ em $[0,1]$ satisfazendo:

1. Para todo $k \in F_{n}, f_{k}\left(x_{k}\right)=1$;

2. Para todo $k \in F_{n}, \operatorname{supp}\left(f_{k}\right) \subseteq \operatorname{supp}\left(g_{k}\right)$;

3. Para todo $k \in F_{n}, \int\left|f_{k}-g_{k}\right| d\left|\mu_{k}\right|<\varepsilon_{k}$;

4. Para todos $m \leq n$ e $i \in I_{n}^{\prime}$ existem $k \in F_{n}$ e $y \in V_{n}^{i}$ tais que $f_{k}(y)=\frac{m}{n}$.

No passo indutivo $n$, suponha definidos $F_{j}$ e $\left\{f_{k}: k \in F_{j}\right\}$, para todo $j<n$. Definiremos $F_{n}$ e $\left\{f_{k}: k \in F_{n}\right\}$.

Para cada $i \in I_{n}^{\prime}$ e $m \leq n$ fixamos $k_{i, m}^{n} \in \omega \backslash \bigcup_{j<n} F_{j}$ tal que $\operatorname{supp}\left(g_{k_{i, m}^{n}}\right) \cap$ $V_{n}^{i} \neq \emptyset$. Podemos assumir que $k_{i, m}^{n} \neq k_{i^{\prime}, m^{\prime}}^{n}$, se $i \neq i^{\prime}$ ou $m \neq m^{\prime}$.

Seja $U_{k_{i, m}^{n}}=\left\{x \in K: g_{k_{i, m}^{n}}(x)>0\right\} \cap V_{n}^{i}$. Como $K$ não tem pontos isolados, $U_{k_{i, m}^{n}}^{i, m}$ é infinito. Logo existe $y_{k_{i, m}^{n}} \in U_{k_{i, m}^{n}}$ tal que $y_{k_{i, m}^{n}} \neq x_{k_{i, m}^{n}}$ e $\left|\mu_{k_{i, m}^{n}}\right|\left(\left\{y_{k_{i, m}^{n}}\right\}\right)<\varepsilon_{k_{i, m}^{n}}$. Usando regularidade das medidas tome $V_{k_{i, m}^{n}} \subseteq$ $U_{k_{i, m}^{n}} \backslash\left\{x_{k_{i, m}^{n}}\right\}$ vizinhança aberta de $y_{k_{i, m}^{n}}$ tal que $\left|\mu_{k_{i, m}^{n}}\right|\left(V_{k_{i, m}^{n}}\right)<\varepsilon_{k_{i, m}^{n}}$.

Defina $F_{n}=\left\{k_{i, m}^{n}: i \in I_{n}^{\prime}, m \leq n\right\}$. Para cada $i \in I_{n}^{\prime}$ e $m \leq n$, usando normalidade de $K$ e o Lema de Urysohn, definimos $f_{k_{i, m}^{n}}: K \longrightarrow$ $[0,1]$ contínua tal que $\left.f_{k_{i, m}^{n}}\right|_{K \backslash V_{k_{i, m}^{n}}}=\left.g_{k_{i, m}^{n}}\right|_{K \backslash V_{k_{i, m}^{n}}}$ e $f_{k_{i, m}^{n}}\left(y_{k_{i, m}^{n}}\right)^{\prime, m}=\frac{m}{n}$. As propriedades 1 a 4 do passo indutivo estão claramente satisfeitas para $F_{n}$ e $\left\{f_{k}: k \in F_{n}\right\}$.

No final da construção indutiva temos definidos $f_{n}$ para todo $n \in \bigcup_{j \in \omega} F_{j}$. Para $n \in \omega \backslash \bigcup_{j \in \omega} F_{j}$ definimos $f_{n}=g_{n}$. Mostraremos que $(a)$ e $(b)$ valem. 
O item (a) segue imediatamente das hipóteses 1 a 3 do passo indutivo. Mostraremos o item $(b)$. Seja $x \in K \backslash D\left(\left(f_{n}\right)_{n \in b}\right)$. Em particular $x \notin D\left(\left(g_{n}\right)_{n \in b}\right)$, uma vez que $\operatorname{supp}\left(f_{n}\right) \subseteq \operatorname{supp}\left(g_{n}\right)$. Mostraremos que $\pi_{K\left(\left(f_{n}\right)_{n \in b}\right), K}^{-1}(x)=\{x\} \times[0,1]$. para isso é suficiente mostrarmos que, para cada $t \in[0,1]$ e $n \in \omega$ existem $j \in \omega$ e $y \in K$ tais que $d(x, y)<\frac{1}{n}$ e $\left|f_{j}(y)-t\right|<\frac{1}{n}$.

Seja $i \in I_{n}$ tal que $x \in V_{n}^{i}$. Como $x \notin D\left(\left(g_{n}\right)_{n \in b}\right)$, temos que $i \in I_{n}^{\prime}$, pois toda vizinhança de $x$ intercepta infinitos suportes de $g_{n}$ 's. Seja $m \leq n$ tal que $\left|t-\frac{m}{n}\right|<\frac{1}{n}$. Pelo item 4 da hipótese indutiva existem $y \in V_{n}^{i} \mathrm{e}$ $j \in \omega$ tais que $f_{j}(y)=\frac{m}{n}$. Como $\operatorname{diam}\left(V_{n}^{i}\right)=\frac{1}{n}$ temos que $d(x, y)<\frac{1}{n}$, como queríamos.

Lema 1.22. Seja $K$ um espaço compacto de peso enumerável sem pontos isolados. Sejam $\left\{X_{n}: n \in \omega\right\}$ e $\left\{Y_{n}: n \in \omega\right\}$ familias de subconjuntos enumeráveis de $K$ tais que $X_{n} \cap Y_{n}=\emptyset$ mas $\bar{X}_{n} \cap \bar{Y}_{n} \neq \emptyset$. Seja $\left\{x_{n}: n \in \omega\right\}$ uma seqüência relativamente discreta em $K$ e disjunta de $\bigcup_{m \in \omega} X_{m} \cup Y_{m}$. Existem funções contínuas $f_{n}: K \longrightarrow[0,1]$ duas a duas disjuntas tais que

(a) Para todo $n \in \omega, f_{n}\left(x_{n}\right)=1$;

(b) Para todo $x \in K \backslash D\left(\left(f_{n}\right)_{n \in \omega}\right), \pi^{-1}\{x\}=\{x\} \times[0,1]$, onde $\pi$ é a projeção de $K\left(\left(f_{n}\right)_{n \in b}\right)$ em $K$;

(c) Para todo $n \in \omega, \overline{X_{n}^{\prime}} \cap \overline{Y_{n}^{\prime}} \neq \emptyset$ em $K\left(\left(f_{n}\right)_{n \in b}\right)$, onde $X_{n}^{\prime}=\left\{\left(x, \Sigma_{n \in b} f_{n}(x)\right)\right.$ : $\left.x \in X_{n}\right\}$ e $Y_{n}^{\prime}=\left\{\left(x, \Sigma_{n \in b} f_{n}(x)\right): x \in Y_{n}\right\}$.

(d) A extensão $K\left(\left(f_{n}\right)_{n \in b}\right)$ é forte.

Demonstração: Seja $\left(g_{n}\right)_{n \in \omega}$ uma seqüência de funções de suportes dois a dois disjuntos tais que $g_{n}\left(x_{n}\right)=1$. Modificaremos $g_{n}$ 's de modo a obtermos itens $b$ ) e $c$ ).

Como $K$ é compacto e tem peso enumerável, $K$ é metrizável. Logo, existem seqüências $\left\{q_{n}^{m}: n \in \omega\right\}$ e $\left\{r_{n}^{m}: n \in \omega\right\}$ de pontos distintos de $X_{m}$ e $Y_{m}$, respectivamente, para cada $m$, e pontos $q^{m} \in K$ tais que $q_{n}^{m} \stackrel{n}{\longrightarrow} q^{m}$ e $r_{n}^{m} \stackrel{n}{\longrightarrow} q^{m}$, para todo $m$.

Observe que, se $q^{m} \in D\left(\left(g_{n}\right)_{n \in b}\right)$, isto é, se existe uma vizinhança de $q^{m}$ que intercepta apenas uma quantidade finita de suportes de $g_{n}$ 's, para $n \in b$, então item $c$ ) será satisfeito para $X_{m}^{\prime}$ e $Y_{m}^{\prime}$, em qualquer extensão 
$K\left(\left(f_{n}\right)_{n \in b}\right)$ tal que $\operatorname{supp}\left(f_{n}\right) \subseteq \operatorname{supp}\left(g_{n}\right)$, para todo $n \in b$, pois, nesse caso, $\Sigma_{n \in b} f_{n}$ é contínua numa vizinhança de $q^{m}$. Portanto podemos assumir que $q^{m} \notin D\left(\left(g_{n}\right)_{n \in b}\right)$, para todo $m \in \omega$. Em particular, $g_{n}\left(q^{m}\right)=0$, para todos $n, m \in \omega$. Como $g_{n}$ 's têm suportes dois a dois disjuntos, $q^{m} \notin \operatorname{supp}\left(g_{n}\right)$, para todos $n, m$.

Vamos construir $\left(f_{n}\right)_{n \in \omega}$ tal que $f_{n}\left(x_{n}\right)=1, \operatorname{supp}\left(f_{n}\right) \subseteq \operatorname{supp}\left(g_{n}\right)$ e $f_{n}\left(q_{i}^{m}\right)=0$, para todo $n \in \omega$, todo $m \in \omega$ e infinitos $i \in \omega$, bem como $f_{n}\left(r_{i}^{m}\right)=0$, para todo $n \in \omega$, todo $m \in \omega$ e infinitos $i \in \omega$. Após mostrarmos o item $(b)$, para termos $\left(q^{m}, 0\right) \in K\left(\left(f_{n}\right)_{n \in \omega}\right)$, isso será suficiente para obtermos $(c)$.

Procedendo por indução em $n$, construiremos conjuntos finitos $F_{n} \subseteq \omega$, funções contínuas $\left\{f_{i}: i \in F_{n}\right\}$ de $K$ em $[0,1]$ e inteiros $\left\{k_{n, m}: m \leq n\right\}$ e $\left\{l_{n, m}: m \leq n\right\}$ tais que

1. se $j \leq n$ então $F_{j} \subseteq F_{n}$;

2. $k_{n, m}>k_{n^{\prime}, m^{\prime}}$ e $l_{n, m}>l_{n^{\prime}, m^{\prime}}$, para todos $m, m^{\prime}, n^{\prime}$ tais que $m \leq n$ e $m^{\prime} \leq n^{\prime}<n$

3. $h_{i} \leq g_{i}$ e $h_{i}\left(x_{i}\right)=1$, para todo $i \in F_{n}$;

4. $q_{k_{j, m}}^{m}, r_{l_{j, m}}^{m} \notin \operatorname{supp}\left(h_{i}\right)$, para todos $i \in F_{n}, m \leq j \leq n$;

5. $q_{k_{j, m}}^{m}, r_{l_{j, m}}^{m} \notin \operatorname{supp}\left(h_{i}\right)$, para todos $i \in \omega \backslash F_{n}, m \leq j \leq n$;

No passo inicial $n=0$ definimos $F_{0}=\emptyset$ e $k_{0,0}=l_{0,0}=0$. Suponha definidos $F_{n},\left\{f_{i}: i \in F_{n}\right\},\left\{k_{n, m}: m \leq n\right\}$ e $\left\{l_{n, m}: m \leq n\right\}$ Construiremos $F_{n+1},\left\{f_{i}: i \in F_{n+1}\right\},\left\{k_{n+1, m}: m \leq n+1\right\}$ e $\left\{l_{n+1, m}: m \leq n+1\right\}$.

Como $\operatorname{supp}\left(h_{i}\right) \subseteq \operatorname{supp}\left(g_{i}\right)$, para todo $i \in F_{n}$, e $q^{m} \notin \operatorname{supp}\left(g_{i}\right)$, para todo $i \in \omega$, para cada $m \leq n+1$ podemos achar inteiros $k_{m}$ e $l_{m}$ tais que, para todos $m^{\prime} \leq j \leq n$ e todo $i \in F_{n}$, temos $h_{i}\left(q_{k_{m}}^{m}\right)=h_{i}\left(r_{l_{m}}^{m}\right)=0, k_{m}>k_{j, m^{\prime}}$ e $l_{m}>l_{j, m^{\prime}}$. Para cada $m \leq n+1$ tome $i_{m} \in \omega \backslash F_{n}$ tal que $g_{i_{m}}\left(q_{k_{m}}^{m}\right) \neq 0$ e $j_{m} \in \omega \backslash F_{n}$ tal que $g_{j_{m}}\left(r_{l_{m}}^{m}\right) \neq 0$, quando existir (se existir, é único, pois $g_{i}$ 's têm suportes disjuntos). Senão tomo $i_{m}$ e $j_{m}$ quaisquer. Notemos que $m \neq m^{\prime}$ não implica, necessariamente, $i_{m} \neq i_{m^{\prime}}$.

Tomamos $F_{n+1}=F_{n} \cup\left\{i_{m}: m \leq n+1\right\} \cup\left\{j_{m}: m \leq n+1\right\}, k_{n+1, m}=k_{m}$ e $l_{n+1, m}=l_{m}$. Para cada $m \leq n+1$ definimos $h_{i_{m}} \leq g_{i_{m}}$ contínua tal que $h_{i_{m}}\left(x_{i_{m}}\right)=1 \mathrm{e}$, para todo $m^{\prime} \leq n+1$, existem vizinhanças abertas $U_{m^{\prime}} \mathrm{e}$ $V_{m^{\prime}}$ de $q_{k_{m^{\prime}}}^{m^{\prime}}$ e $r_{k_{m^{\prime}}}^{m^{\prime}}$, respectivamente, tais que $\left.h_{i_{m}}\right|_{U_{m^{\prime}}}=\left.h_{i_{m}}\right|_{V_{m^{\prime}}}=0$. Para isso usamos que $x_{i} \neq q_{j}^{m}$, para todos $i, j, m$, e aplicamos Lema de Urysohn. 
A definição de $h_{j_{m}}$ é análoga. As propriedades de 1 a 5 serão claramente preservadas para o passo $n+1$.

No final da construção, definimos $h_{i}=g_{i}$, para todo $i \in \bigcup_{n \in \omega} F_{n}$.

Usando o Lema 1.21 construímos $f_{n}$ 's, $\operatorname{com} \operatorname{supp}\left(f_{n}\right) \subseteq \operatorname{supp}\left(h_{n}\right)$, satisfazendo item $(b)$ e presevando item $(a)$. Como $\operatorname{supp}\left(f_{n}\right) \subseteq \operatorname{supp}\left(h_{n}\right)$, para todo $n$, itens 4 e 5 são preservados para $f_{n}$ no lugar de $h_{n}$. Como, de $(b)$ segue que $\left(q^{m}, 0\right) \in K\left(\left(f_{n}\right)_{n \in \omega}\right.$, pois assumimos que $q^{m} \notin D\left(\left(f_{n}\right)_{n \in \omega}\right)$, por 1 a 5 concluímos que $\left(q^{m}, 0\right) \in \bar{X}_{m}^{\prime} \cap \bar{Y}_{m}^{\prime}$, concluindo $(c)$.

O item $d$ ) segue do item $b)$, pois $\Sigma_{n \in b} f_{n}$ é contínua em $D\left(\left(f_{n}\right)_{n \in b}\right)$ e, portanto, para $x \in D\left(\left(f_{n}\right)_{n \in b}\right)$ temos $\left(x, \Sigma_{n \in b} f_{n}(x)\right) \in K\left(\left(f_{n}\right)_{n \in b}\right)$. Para $x \notin D\left(\left(f_{n}\right)_{n \in b}\right)$, pelo item $\left.b\right)$ concluímos $\left(x, \Sigma_{n \in b} f_{n}(x)\right) \in K\left(\left(f_{n}\right)_{n \in b}\right)$.

Lema 1.23. Seja $K$ um espaço compacto de peso enumerável sem pontos isolados. Dados

a) Uma seqüência $\left(f_{n}: n \in \omega\right)$ de funções contínuas, duas a duas disjuntas de $K$ em $[0,1]$;

b) Uma seqüência $\left(x_{n}: n \in \omega\right)$ em $K$;

c) $\operatorname{Um} \varepsilon>0$;

d) Uma seqüência limitada $\left(\mu_{n}: n \in \omega\right)$ de medidas em $K$ tal que $\left|\int f_{n} d \mu_{n}\right|>$ $\varepsilon$, para todo $n \in \omega$.

existem $\delta>0, a \subseteq \omega$ infinito e funções $f_{n}^{\prime}: K \longrightarrow[0,1], \operatorname{com} \operatorname{supp}\left(f_{n}^{\prime}\right) \subseteq$ $\operatorname{supp}\left(f_{n}\right)$, tais que, para todo $b \subseteq a$

e) $\left|\int f_{n}^{\prime} d \mu_{n}\right|>\delta$ e $\Sigma\left\{\int f_{m}^{\prime} d\left|\mu_{n}\right|: m \neq n, m \in a\right\}<\delta / 3$, para todo $n \in a$;

f) $L=K\left(\left(f_{n}^{\prime}\right)_{n \in b}\right)$ é uma extensão forte tal que, para todo $x \in K, \pi_{L, K}^{-1}[\{x\}]$ é unitário ou igual a $\{x\} \times[0,1]$;

g) $\Delta\left(\left(f_{n}^{\prime}\right)_{n \in b}\right)$ é unitário ou é disjunto de $\left\{x_{n}: n \in \omega\right\}$.

Demonstração: Dados $\left(A_{n}\right)_{n \in \omega}$ abertos dois a dois disjuntos de $K$, defina $\Delta\left(\left(A_{n}\right)_{n \in \omega}\right)=\left\{x \in K\right.$ : toda vizinhança $U$ de $x$ intercepta infinitos $A_{n}$ 's $\}$.

Defina $A_{n}=\left\{x \in K: f_{n}(x)>0\right\}$, para cada $n \in \omega$. Está claro que $\Delta\left(\left(A_{n}\right)_{n \in \omega}\right)=\Delta\left(\left(f_{n}\right)_{n \in \omega}\right)$. 
A próxima afirmação é uma adaptação para o caso não 0-dimensional de parte da demonstração do Lema 7 de [Ko3]. Notamos que, se $A_{n}$ 's são abertos-fechados, $\Delta\left(\left(A_{n}\right)_{n \in \omega}\right)=\overline{\bigcup_{n \in \omega} A_{n}}-\bigcup_{n \in \omega} A_{n}$, conforme o enunciado do Lema 7 de [Ko3].

Afirmação 1.23.1. Existem $N_{1} \subseteq \omega$ infinito, $\delta>0$ e abertos $A_{n}^{\prime}$, para $n \in N_{1}$ tais que $A_{n}^{\prime} \subseteq A_{n},\left|\mu_{n}\left(A_{n}^{\prime}\right)\right|>\delta e$

1. $\Delta\left(\left(A_{n}^{\prime}\right)_{n \in N_{1}}\right)$ é unitário, ou

2. $x_{m} \notin \Delta\left(\left(A_{n}^{\prime}\right)_{n \in N_{1}}\right)$, para todo $m \in N_{1}$.

Caso 1: Existe $\delta^{\prime}>0$ e $x \in K$ tais que, para toda vizinhança aberta $V$ de $x$ e para todo $m \in \omega$ existe $k>m$ tal que $\left|\mu_{k}\right|\left(A_{k} \cap V\right)>\delta^{\prime}$.

Como $K$ é metrizável, existe $\left(V_{n}\right)_{n \in \omega}$ um sistema fundamental decrescente de vizinhanças de $x$. Construímos, por indução, $N_{1} \subseteq \omega$ infinito e inteiros $k_{n}$ distintos tais que $\left|\mu_{n}\right|\left(A_{n} \cap V_{k_{n}}\right)>\delta^{\prime}$, para todo $n \in N_{1}$. Tomamos $A_{n}^{\prime}=$ $A_{n} \cap V_{k_{n}}$. Usando a definição de variação de medida podemos assumir que $\left|\mu_{n}\left(A_{n}^{\prime}\right)\right|>\delta^{\prime}$, para todo $n \in N_{1}$, trocando $\delta^{\prime}$ por $\frac{\delta^{\prime}}{2}$. Teremos $\Delta\left(\left(A_{n}^{\prime}\right)_{n \in \omega}\right)=$ $\{x\}$, pois toda vizinhança de $x$ contém todos, exceto finitos, $A_{n}^{\prime}$ 's, já que $\left(V_{k_{n}}\right)_{n \in N_{1}}$ é um sistema fundamental de vizinhanças de $x$. A afirmação vale, tomando $\delta=\delta^{\prime}$.

Caso 2: Não ocorre caso 1.

Para cada $n \in \omega$ e $\delta^{\prime}>0$ existem $m\left(n, \delta^{\prime}\right) \in \omega$ e uma vizinhança aberta $V\left(n, \delta^{\prime}\right)$ de $x_{n}$ tais que

$$
\left|\mu_{k}\right|\left(A_{k} \cap V\left(n, \delta^{\prime}\right)\right)<\delta^{\prime}
$$

para todo $k>m\left(n, \delta^{\prime}\right)$. Pela regularidade de $K$ podemos assumir que

$$
\left|\mu_{k}\right|\left(A_{k} \cap \overline{V\left(n, \delta^{\prime}\right)}\right)<\delta^{\prime}
$$

para todo $k>m\left(n, \delta^{\prime}\right)$. Para isso basta substituirmos $V\left(n, \delta^{\prime}\right)$ por $V^{\prime}\left(n, \delta^{\prime}\right)$ tal que $x_{n} \in V^{\prime}\left(n, \delta^{\prime}\right) \subseteq \overline{V^{\prime}\left(n, \delta^{\prime}\right)} \subseteq V\left(n, \delta^{\prime}\right)$.

Escolhemos por indução uma seqüência estritamente crescente $\left(k_{n}\right)_{n \in \omega}$ de inteiros tais que $k_{n}>m\left(j, \varepsilon / 2^{j+2}\right)$, para todo $j<n$. Considere

$$
A_{k_{n}}^{\prime}=A_{k_{n}} \backslash \bigcup\left\{\overline{V\left(j, \varepsilon / 2^{j+2}\right)}: j<n\right\}
$$


Por $(*)$ temos $\left|\mu_{k_{n}}\right|\left(A_{k_{n}} \cap \overline{V\left(j, \varepsilon / 2^{j+2}\right)}\right)<\frac{\varepsilon}{2^{j+2}}$, para $j<n$, e, portanto,

$$
\left|\mu_{k_{n}}\right|\left(A_{k_{n}}^{\prime}\right)>\varepsilon / 2 \text {. }
$$

Tome $N_{1}=\left\{k_{n}: n \in \omega\right\}$ e $\delta=\varepsilon / 2$. Como $V\left(n, \varepsilon / 2^{n+2}\right)$ é disjunto de $A_{k_{i}}^{\prime}$, para $i>n$, temos que $x_{n} \notin \Delta\left(\left(A_{n}^{\prime}\right)_{n \in N_{1}}\right)$, concluindo a demonstração da afirmação.

Fixamos, para cada $n \in N_{1}, \delta_{n}>\delta$ tal que $\left|\mu_{n}\left(A_{n}^{\prime}\right)\right|>\delta_{n}$. Usando a regularidade das medidas $\mu_{n}$ achamos $B_{n} \subseteq A_{n}^{\prime}$ fechados tais que $\left|\mu_{n}\left(B_{n}\right)\right|>$ $\delta_{n}$ e $\left|\mu_{n}\right|\left(B_{n}-A_{n}^{\prime}\right)<\delta_{n}-\delta$. Como $K$ é normal, usando o Teorema de Tietze achamos $f_{n}^{\prime}$ tais que $\left.f_{n}^{\prime}\right|_{B_{n}}=1$ e $\left.f_{n}^{\prime}\right|_{K-A_{n}^{\prime}}=0$. Temos que $\left|\int f_{n}^{\prime} d \mu_{n}\right|>\delta$ e $\operatorname{supp}\left(f_{n}^{\prime}\right) \subseteq \operatorname{supp}\left(f_{n}\right)$. Note que $\Delta\left(\left(f_{n}^{\prime}\right)_{n \in N_{1}}\right) \subseteq \Delta\left(\left(A_{n}^{\prime}\right)_{n \in N_{1}}\right)$, de onde temos item $g$ ).

Pelo Lema de Rosenthal, usando que $\int f_{m}^{\prime} d\left|\mu_{n}\right|<\left|\mu_{n}\right|\left(A_{m}\right)$, achamos $N_{2} \subseteq N_{1}$ de modo a satisfazer a segunda parte do item e). Para obtermos item $f$ ), usamos o Lema 1.21 para modificamos $f_{n}^{\prime}$ 's de forma a obter $f$ ) preservando $b$ ). Lema 1.19 para obtermos uma subseqüência de $\left(f_{n}\right)_{n \in N_{1}}$ tal que a extensão seja forte. Item $g$ ) será preservado, pois não aumentamos $\operatorname{supp}\left(f_{n}^{\prime}\right)$.

\subsection{Axioma $\diamond$}

Nesta seção iremos apresentar a definição e alguns resultados básicos do axioma $\diamond$ (lê-se diamante).

Definição 1.24. Dizemos que um subconjunto $C$ de $\omega_{1}$ é fechado ilimitado se é ilimitado e $\sup B \in C$, para todo $B \subseteq C$ enumerável. Dizemos que um subconjunto $S$ de $\omega_{1}$ é estacionário se intercepta todo fechado ilimitado.

Lema 1.25. A intersecção de uma família enumerável de fechados ilimitados de $\omega_{1}$ é um conjunto fechado ilimitado. Em particular, se $S$ é estacionário e $C$ é fechado ilimitado, então $S \cap C$ é estacionário.

Para a demonstração do Lema 1.25 indicamos [Ku], Capítulo II, Lema 6.8 . 
Axioma $\diamond$ Existe uma seqüência $\left(X_{\alpha}\right)_{\alpha \in \omega_{1}}$ tal que $X_{\alpha} \subseteq \alpha$ e, para todo $X \subseteq \omega_{1}$, o conjunto $\left\{\alpha \in \omega_{1}: X \cap \alpha=X_{\alpha}\right\}$ é estacionário.

A seqüência $\left(X_{\alpha}\right)_{\alpha \in \omega_{1}}$ é chamada $\diamond$-seqüência.

$\mathrm{O}$ axioma $\diamond$ é relativamente consistente com ZFC, valendo no modelo construtível. Para maiores referências vide [Ku], [Je] e [Ve].

Como uma simples aplicação de $\diamond$ vejamos que este implica $\mathrm{CH}$.

Lema 1.26. $\diamond \rightarrow C H$.

Demonstração: Se $X \subseteq \omega$, como conjuntos estacionários são ilimitados (pois $\left\{\alpha<\omega_{1}: \alpha>\beta\right\}$ é fechado ilimitado), temos que existe $\alpha>\omega$ tal que $X=X \cap \alpha=X_{\alpha}$. Logo $\left(X_{\alpha}\right)_{\alpha<\omega_{1}}$ contém todos os subconjuntos de $\omega$.

Lema 1.27. O axioma $\diamond$ implica:

a) $S e\left(B_{\alpha}\right)_{\alpha<\omega_{1}}$ é uma seqüência de conjuntos de tamanho $\omega_{1}$, existe uma seqüência $\left\{x_{\alpha}: \alpha<\omega_{1}\right\}$ tal que $x_{\alpha} \in \Pi_{\beta<\alpha} B_{\beta}$ e, para todo $x \in$ $\Pi_{\alpha<\omega_{1}} B_{\alpha}$ o conjunto $\left\{\alpha<\omega_{1}: x \mid \alpha=x_{\alpha}\right\}$ é estacionário;

b) Existe uma seqüência $\left\{x_{n}(\alpha): n \in \omega, \alpha<\omega_{1}\right\}$ tal que $x_{n}(\alpha) \in[0,1]^{\alpha}$ e, para toda seqüência $\left(x_{n}\right)_{n \in \omega}$ de pontos de $[0,1]^{\omega_{1}}$, o conjunto $\left\{\alpha \in \omega_{1}\right.$ : $\left.\forall n \in \omega\left(x_{n} \mid \alpha=x_{n}(\alpha)\right)\right\}$ é estacionário;

c) Existe uma seqüência $\left(x_{\alpha}\right)_{\alpha<\omega_{1}}$, com $x_{\alpha} \in[0,1]^{\alpha \times \alpha}$, tal que, para todo $x \in[0,1]^{\omega_{1} \times \omega_{1}}$, o conjunto $\left\{\alpha<\omega_{1}: x \mid \alpha \times \alpha=x_{\alpha}\right\}$ é estacionário.

d) Existe uma seqüência $\left\{A_{\alpha}: \alpha<\omega_{1}\right\}$ de subconjuntos de $\omega_{1}$ tal que, se $\left(z_{\beta}\right)_{\beta \in \omega_{1}}$ é uma seqüência de pontos de $[0,1]^{\omega_{1}}$, o conjunto $\left\{\alpha \in \omega_{1}\right.$ : $\left.\left\{z_{\beta} \mid \alpha: \beta<\alpha\right\}=A_{\alpha}\right\}$ é estacionário.

Demonstração: Para demonstrar $a)$ tome $\left(X_{\alpha}\right)_{\alpha<\omega_{1}}$ uma $\diamond$-seqüência. Seja $\left\{\xi_{\alpha}: \alpha<\omega_{1}\right\}$ uma seqüência crescente em $\omega_{1}$ definida da seguinte forma: $\xi_{\alpha+1}=\xi_{\alpha}+\omega$ e $\xi_{\alpha}=\sup \left\{\xi_{\alpha^{\prime}}: \alpha^{\prime}<\alpha\right\}$ para $\alpha$ limite.

Para cada $\alpha<\omega_{1}$ seja $\phi_{\alpha}: \mathcal{P}\left(\left[\xi_{\alpha}, \xi_{\alpha+1}\right)\right) \rightarrow B_{\alpha}$ uma função bijetora (existe, pois $\diamond \rightarrow \mathrm{CH}$ ). Definimos $x_{\alpha} \in \Pi_{\beta<\alpha} B_{\beta}$ dado por

$$
x_{\alpha}(\beta)=\phi_{\beta}\left(X_{\xi_{\alpha}} \cap\left[\xi_{\beta}, \xi_{\beta+1}\right]\right),
$$

para todo $\beta<\omega_{1}$. 
Mostraremos que a seqüência $\left(x_{\alpha}\right)_{\alpha<\omega_{1}}$ satisfaz $\left.a\right)$. Seja $x \in \prod_{\alpha<\omega_{1}} B_{\alpha}$. Seja $X=\cup\left\{\phi_{\alpha}^{-1}(x(\alpha)): \alpha<\omega_{1}\right\}$. Temos $x \mid \alpha=x_{\alpha}$ se, e somente se, $X \cap \xi_{\alpha}=X_{\xi_{\alpha}}$.

Pelo Lema 1.25 temos que $\left\{\alpha<\omega_{1}: X \cap \xi_{\alpha}=X_{\xi_{\alpha}}\right\}=\left\{\beta<\omega_{1}: X \cap \beta=\right.$ $\left.X_{\beta}\right\} \cap\left\{\xi_{\alpha}: \alpha<\omega_{1}\right\}$ é estacionário. Mas

$$
\left\{\alpha<\omega_{1}: X \cap \xi_{\alpha}=X_{\xi_{\alpha}}\right\}=\left\{\alpha<\omega_{1}: x \mid \alpha=x_{\alpha}\right\}
$$

concluindo o item $a$ ).

Para o item $b$ ) tomamos $B_{\alpha}=[0,1]^{\omega}$ e usamos o item $\left.a\right)$.

Para mostrarmos c), usamos o item $a$ ) para $B_{\alpha}=[0,1]^{\{\alpha\} \times(\alpha+1)} \cup[0,1]^{(\alpha+1) \times\{\alpha\}}$. Há uma identificação natural de $\Pi_{\beta<\alpha} B_{\beta} \operatorname{com}[0,1]^{\alpha \times \alpha}$, associando cada $f \in \Pi_{\beta<\alpha} B_{\beta} \operatorname{com} x=\bigcup_{\beta<\alpha} f(\beta) \in[0,1]^{\alpha \times \alpha}$. Assim, basta tomarmos uma seqüência $x_{\alpha} \in[0,1]^{\alpha \times \alpha}$ como em $\left.a\right)$. Se identificarmos $f \in \prod_{\alpha<\omega_{1}} B_{\alpha}$ com $x \in[0,1]^{\omega_{1} \times \omega_{1}}$, temos que $f \mid \alpha$ corresponde a $\left.x\right|_{\alpha \times \alpha}$, concluindo $\left.c\right)$.

Mostraremos $d)$. Fixe $\left(x_{\alpha}\right)_{\alpha<\omega_{1}}$ como no item $c$ ). Para cada $\beta<\alpha<\omega_{1}$ definimos $x_{\beta, \alpha} \in[0,1]^{\alpha}$ por $x_{\beta, \alpha}(\gamma)=x_{\alpha}(\beta, \gamma)$, para $\gamma<\alpha$. Seja $A_{\alpha}=$ $\left\{x_{\beta, \alpha}: \beta<\alpha\right\}$. Para uma seqüência $\left(z_{\beta}\right)_{\beta<\omega_{1}}$ em $[0,1]^{\omega_{1}}$ associamos um $x \in[0,1]^{\omega_{1} \times \omega_{1}}$ dado por $x(\beta, \gamma)=z_{\beta}(\gamma)$. Logo

$$
\left\{\alpha<\omega_{1}:\left\{z_{\beta} \mid \alpha: \beta<\alpha\right\}=A_{\alpha}\right\} \supseteq\left\{\alpha<\omega_{1}: x \mid \alpha \times \alpha=x_{\alpha}\right\},
$$

que é estacionário, pelo item c). Da definição de conjuntos estacionários segue imediatamente que superconjuntos de conjuntos estacionários são estacionários. Portanto concluímos item $d$ ).

Lema 1.28. Seja $Y \subseteq[0,1]^{\omega_{1}}$ e seja $\left(x_{\alpha}\right)_{\alpha<\omega_{1}}$ uma seqüência densa em $Y$. Então $\left\{\alpha<\omega_{1}:\left(x_{\beta} \mid \alpha\right)_{\beta<\alpha}\right.$ é denso em $\left.\pi_{\alpha}[Y]\right\}$ é fechado ilimitado em $\omega_{1}$.

Demonstração: Para mostrarmos o lema basta mostrarmos a seguinte afirmação:

Afirmação 1.28.1. Seja $\left(\gamma_{n}\right)_{n \in \omega} e\left(\alpha_{n}\right)_{n \in \omega}$ seqüências crescentes de ordinais que têm supremos $\gamma$ e $\alpha$, respectivamente, tais que, para cada $n \in \omega$, $\left(x_{\beta} \mid \alpha_{n}\right)_{\beta<\gamma_{n}}$ é denso em $\pi_{\alpha_{n}}[Y]$. Então $\left(x_{\beta} \mid \alpha\right)_{\beta<\gamma}$ é denso em $\pi_{\alpha}[Y]$.

Para mostrarmos a afirmação, suponha que exista $U$ um aberto elementar de $[0,1]^{\alpha}$ tal que $U \cap \pi_{\alpha}[Y] \neq \emptyset$ e $x_{\beta} \mid \alpha \notin U$, para todo $\beta<\gamma$. Tome $n \in \omega$ tal que $\alpha_{n}$ contenha as coordenadas que determinam $U$. Temos que $\pi_{\alpha_{n}}[U]$ 
é um aberto de $[0,1]^{\alpha_{n}}$ que intercepta $\pi_{\alpha_{n}}[Y]$ e é disjunto de $\left(x_{\beta} \mid \alpha_{n}\right)_{\beta<\gamma_{n}}$, contradizendo a hipótese e provando a afirmação.

Da afirmação concluímos que $\left\{\alpha<\omega_{1}:\left(x_{\beta} \mid \alpha\right)_{\beta<\alpha}\right.$ é denso em $\left.\pi_{\alpha}[Y]\right\}$ é fechado, tomando o caso particular $\alpha_{n}=\gamma_{n}$. Para mostrar que é ilimitado, tome $\alpha_{0} \in \omega_{1}$. Pela continuidade de $\pi$ temos que $\left(x_{\beta} \mid \alpha_{0}\right)_{\beta<\omega_{1}}$ é denso em $\pi_{\alpha_{0}}[Y]$. Como $\pi_{\alpha_{0}}[Y]$ tem peso enumerável, para cada vizinhança aberta de uma base enumerável de $\pi_{\alpha_{0}}[Y]$ tomamos algum $x_{\beta} \mid \alpha_{0}$ pertencente a ela. Assim obtemos $\alpha_{1}$, que podemos supor maior que $\alpha_{0}$, tal que $\left(x_{\beta} \mid \alpha_{0}\right)_{\beta<\alpha_{1}}$ é denso em $\pi_{\alpha_{0}}[Y]$. Por indução, construímos uma seqüência crescente $\alpha_{n}$ tal que $\left(x_{\beta} \mid \alpha_{n}\right)_{\beta<\alpha_{n+1}}$ é denso em $\pi_{\alpha_{n}}[Y]$. Da observação anterior, tomando $\alpha=\sup \left\{\alpha_{n}: n \in \omega\right\}$, temos que $\left(x_{\beta} \mid \alpha\right)_{\beta<\alpha}$ é denso em $\pi_{\alpha}[Y]$. 
Preliminares 


\section{Capítulo 2}

\section{Quocientes de espaços indecomponíveis da forma $C(K)$}

Respondendo a uma pergunta apresentada no final de [Ko2], neste capítulo construímos, assumindo $\diamond$, um espaço topológico $K$ compacto e conexo tal que para todo fechado $L \subseteq K$ o espaço $C(L)$ tem poucos operadores. Como subespaços topológicos de $K$ induzem quocientes de $C(K)$, concluímos que tal espaço tem pelo menos contínuo quocientes da forma $C(L)$ indecomponíveis.

Sabemos que $C(\beta \mathbb{N})=l_{\infty}$ não contém poucos operadores, pois, por exemplo, $l_{\infty}=l_{\infty} \oplus \mathbb{R}$, e em Ko2], mostra-se que espaços de Banach com poucos operadores não são isomorfos aos hiperplanos. Portanto o compacto $K$ construído na Seção 2.1 não contém um subespaço homeomorfo a $\beta \mathbb{N}$. Também $K$ não contém uma seqüência convergente não trivial, pois senão teríamos $c_{0}$ complementado em $C(K)$. Portanto $K$ responde positivamente ao problema de Efimov, sobre a existência de um compacto que não contém seqüências convergentes não-trivias nem $\beta \mathbb{N}$ como subespaço. O problema de Efimov já foi resolvido positivamente em 1975 por Fedorchuk (vide [Fed]), assumindo CH. O problema de Efimov ainda permanece em aberto em ZFC.

Podemos perguntar se todo compacto $K$ tal que $C(K)$ é indecomponível responde afirmativamente ao problema de Efimov. Mostramos que não. Assumindo $\mathrm{CH}$, na Seção 2.2 construímos um espaço $C(K)$ indecomponível tal que $K$ contém $\beta \mathbb{N}$ homeomorficamente. Em particular, $C(K)$ contém $l_{\infty}$ como quociente. Não sabemos se o espaço $C(K)$ construído na Seção 2.1 contém $l_{\infty}$ como quociente. Talagrand mostrou ([Ta]) que $C(K)$ contém $l_{\infty}$ como quociente se, e somente se, $B_{C(K)^{*}}$ contém $\beta \mathbb{N}$ homeomorficamente. 


\subsection{Um espaço $C(K)$ com muitos quocientes indecomponíveis}

O Teorema seguinte é uma versão do Teorema 5.1 de [Ko2]. A diferença fundamental da versão aqui utilizada é que o item $g$ ) é obtido para qualquer seqüência $\left(x_{n}\right)_{n \in \omega}$ em $K$, enquanto na versão de [Ko2] tal seqüência deve ser tomada em um denso enumerável previamente fixado. Com isso conseguimos transferir a propriedade de $C(K)$ ter poucos operadores para todo subespaço fechado de $K$, mas precisamos do axioma $\diamond$, para enumerar as seqüências de $K$ de uma maneira conveniente (veja Seção 1.4 sobre o axioma $\diamond$ ). Para obtermos item $g$ ) para seqüências quaisquer, foi necessário modificar as funções $f_{n}$ 's para adicionar supremos, utilizando o Lema 1.23.

Teorema 2.1. Assuma $\diamond$. Existe um espaço compacto e conexo $K$ tal que:

i) dados

a) Uma seqüência $\left(f_{n}: n \in \omega\right)$ de funções contínuas, duas a duas disjuntas, de $K$ em $[0,1]$;

b) Uma seqüência $\left(x_{n}: n \in \omega\right)$ relativamente discreta de pontos distintos de $K$ tal que $f_{m}\left(x_{n}\right)=0$, para todos $n, m \in \omega$;

c) $U m \varepsilon>0$;

d) Uma seqüência limitada $\left(\mu_{n}: n \in \omega\right)$ de medidas em $K$ tal que $\left|\int f_{n} d \mu_{n}\right|>\varepsilon$, para todo $n \in \omega$.

existem $\delta>0, b \subseteq a \subseteq \omega$ infinitos e funções $f_{n}^{\prime}$, com $\operatorname{supp}\left(f_{n}^{\prime}\right) \subseteq$ $\operatorname{supp}\left(f_{n}\right)$ tais que

e) $\left|\int f_{n}^{\prime} d \mu_{n}\right|>\delta$ e $\Sigma\left\{\int f_{m}^{\prime} d\left|\mu_{n}\right|: m \neq n, m \in a\right\}<\delta / 3$, para todo $n \in a$;

f) $\left(f_{n}^{\prime}\right)_{n \in b}$ tem supremo em $C(K)$;

g) $\overline{\left\{x_{n}: n \in b\right\}} \cap \overline{\left\{x_{n}: n \in a \backslash b\right\}} \neq \emptyset$.

ii) Se $L$ é um subespaço fechado de $K$ e $V_{1}$ e $V_{2}$ são abertos disjuntos de $L$ tais que $\bar{V}_{1} \cap \bar{V}_{2} \neq \emptyset$, então $\bar{V}_{1} \cap \bar{V}_{2}$ tem pelo menos dois elementos. 
Demonstração: Para cada $\alpha \leq \omega_{1}$ considere $\mathcal{B}_{\alpha}$ a base de abertos elementares de extremos racionais do espaço $[0,1]^{\alpha}$. Podemos identificar as medidas de Radon de $[0,1]^{\alpha}$ com funções de $\mathcal{B}_{\alpha}$ em $\mathbb{R}$ (Lema 1.1).

Sejam Par, Impar os conjuntos dos ordinais pares e ímpares, respectivamente, de $\omega_{1}$, lembrando que $\alpha$ é um ordinal par se é da forma $\beta+n$, para $\beta$ ordinal limite e $n$ par, e ímpar caso contrário.

Se $X \subseteq \omega_{1}$ é não-enumerável, existe um isomorfismo de ordem entre $X$ e $\omega_{1}$, ordenando $X$ com a restrição da ordem de $\omega_{1}$. Seja $\sigma: X \longrightarrow \omega_{1}$ esse isomorfismo. Diremos que um conjunto $C \subseteq X$ é fechado ilimitado em $X$ se $\{\sigma(\alpha): \alpha \in X\}$ é fechado ilimitado em $\omega_{1}$. Diremos que $S \subseteq X$ é estacionário em $X$ se intercepta todo fechado ilimitado em $X$. Da mesma forma será quando aplicarmos o axioma $\diamond$ em $X$, isto é, identificaremos $X$ com $\omega_{1}$. Usaremos essa terminologia para Par e Impar.

Usando $\diamond$ e os Lemas 1.27 e 1.25 fixamos enumerações $\left\{f_{n}(\alpha): n \in \omega\right\}$, $\varepsilon(\alpha),\left\{\mu_{n}(\alpha): n \in \omega\right\},\left\{x_{n}(\alpha): n \in \omega\right\}$, para $\alpha \in$ Par, tais que

A.1. $\left\{f_{n}(\alpha): n \in \omega\right\}$ são funções contínuas de $[0,1]^{\omega_{1}}$ em $[0,1]$;

A.2. $\varepsilon(\alpha)>0$

A.3. $\left(\mu_{n}(\alpha)\right)_{n \in \omega}$ é uma seqüência limitada de funções de $\mathcal{B}_{\alpha}$ em $\mathbb{R}$;

A.4. $\left(x_{n}(\alpha)\right)_{n \in \omega}$ é uma seqüência de pontos de $[0,1]^{\alpha}$;

$\mathrm{e}, \operatorname{dados} \beta<\omega_{1} \mathrm{e}$

B.1. uma seqüência $\left\{f_{n}: n \in \omega\right\}$ de funções contínuas de $[0,1]^{\omega_{1}}$ em $[0,1]$;

B.2. $\operatorname{um} \varepsilon>0$;

B.3. uma seqüência $\left\{\mu_{n}: n \in \omega\right\}$ limitada de funções de $\mathcal{B}_{\omega_{1}}$ em $\mathbb{R}$ que representam medidas de Radon;

B.4. uma seqüência $\left(x_{n}\right)_{n \in \omega}$ relativamente discreta em $[0,1]^{\omega_{1}}$;

existe $\alpha>\beta$, com $\alpha \in P$ ar, tal que

C.1. $f_{n}(\alpha)=f_{n}$, para todo $n$;

C.2. $\varepsilon(\alpha)=\varepsilon$; 
C.3. $\mu_{n}(\alpha)=\mu_{n} \mid \mathcal{B}_{\alpha}$, para todo $n$;

C.4. $x_{n}(\alpha)=x_{n} \mid \alpha$, para todo $n$.

Usando $\diamond$ para os ordinais ímpares, fixamos seqüências $\left(U_{\alpha}, V_{\alpha}, A_{\alpha}, B_{\alpha}\right)_{\alpha \in \text { Impar }}$, onde

D.1. $U_{\alpha}$ e $V_{\alpha}$ são uniões enumeráveis de abertos elementares de $[0,1]^{\omega_{1}}$ tais que $U_{\alpha} \cap V_{\alpha}=\emptyset$ e $\bar{U}_{\alpha} \cap \bar{V}_{\alpha} \neq \emptyset$;

D.2. $A_{\alpha}$ e $B_{\alpha}$ são subconjuntos enumeráveis de $[0,1]^{\alpha}$;

e, dados

E.1. $U$ e $V$ uniões enumeráveis de abertos elementares de $[0,1]^{\omega_{1}}$ tais que $U \cap V=\emptyset$ е $\bar{U} \cap \bar{V} \neq \emptyset$;

E.2. $\left(x_{\beta}\right)_{\beta<\omega_{1}}$ e $\left(y_{\beta}\right)_{\beta<\omega_{1}}$ seqüências em $[0,1]^{\omega_{1}}$;

o conjunto

$$
\begin{gathered}
\left\{\alpha \in \text { Impar }: U_{\alpha}=U, V_{\alpha}=V,\left\{x_{\beta} \mid \alpha: \beta \in \text { Impar } \cap \alpha\right\}=A_{\alpha},\right. \\
\left.\left\{y_{\beta} \mid \alpha: \beta \in \text { Impar } \cap \alpha\right\}=B_{\alpha}\right\}
\end{gathered}
$$

é estacionário em Impar.

Seja $\alpha \in$ Impar. Se $\overline{\pi_{\alpha}\left[U_{\alpha}\right] \cap A_{\alpha}} \cap \overline{\pi_{\alpha}\left[V_{\alpha}\right] \cap B_{\alpha}} \neq \emptyset$ fixamos $\left(x_{n}(\alpha)\right)_{\alpha \in \omega}$ tal que $x_{n}(\alpha) \stackrel{n \in \omega}{\rightarrow} z$, para algum $z \in \overline{\pi_{\alpha}\left[U_{\alpha}\right] \cap A_{\alpha}} \cap \overline{\pi_{\alpha}\left[V_{\alpha}\right] \cap B_{\alpha}}$ e

$$
\begin{gathered}
\left\{x_{n}(\alpha): n \in 2 \omega\right\} \subseteq A_{\alpha} ; \\
\left\{x_{n}(\alpha): n \in \omega \backslash 2 \omega\right\} \subseteq B_{\alpha} .
\end{gathered}
$$

Se $\overline{\pi_{\alpha}\left[U_{\alpha}\right] \cap A_{\alpha}} \cap \overline{\pi_{\alpha}\left[V_{\alpha}\right] \cap B_{\alpha}}=\emptyset$ tomamos $\left(x_{n}(\alpha)\right)_{n \in \omega}$ qualquer seqüência em $A_{\alpha} \cup B_{\alpha}$.

Dizemos que uma seqüência de fechados $\left(F_{n}\right)_{n \in \omega}$ converge a um ponto $x$ se para toda vizinhança $U$ de $x$ temos $F_{n} \subseteq U$, para todos, exceto finitos, $n \in \omega$.

Construiremos por indução espaços compactos $\left(K_{\alpha}\right)_{\alpha<\omega_{1}}$, com $K_{\alpha} \subseteq$ $[0,1]^{\alpha}$, seqüências $P_{\alpha}=\left\{\left(L_{(\beta, i)}^{\alpha}, R_{(\beta, i)}^{\alpha}, z_{(\beta, i)}^{\alpha}\right):(\beta, i) \in \alpha \times\{0,1\}\right\}$, com $L_{(\beta, i)}^{\alpha}, R_{(\beta, i)}^{\alpha} \subseteq \omega$ disjuntos e $z_{(\beta, i)}^{\alpha} \in K_{\alpha}$, e fechados $F_{n}^{\beta}(\alpha) \subseteq K_{\alpha}$, para $\beta \leq \alpha$.

Uma vez definido $K_{\alpha}$, para cada $\beta \leq \alpha$ definimos $F_{n}^{\beta}(\alpha)=\pi_{K_{\alpha}, K_{\beta}}^{-1}\left[\left\{x_{n}(\beta)\right\}\right]$.

Suponha construídos $\left(K_{\gamma}\right)_{\gamma<\alpha}$ e $\left(P_{\gamma}\right)_{\gamma<\alpha}$. Temos as seguintes hipóteses indutivas, para todo $\gamma<\alpha$, 
F.1. para todo $(\beta, i) \in \gamma \times\{0,1\}, \lim _{n \in L_{(\beta, i)}^{\gamma}} F_{n}^{\beta}(\gamma)=\lim _{n \in R_{(\beta, i)}^{\gamma}} F_{n}^{\beta}(\gamma)=$ $z_{(\beta, i)}^{\gamma}$.

F.2. para todos $\beta<\gamma^{\prime}<\gamma$ e $i \in\{0,1\}, \pi_{\gamma^{\prime}}\left[K_{\gamma}\right]=K_{\gamma^{\prime}}$ e $z_{(\beta, i)}^{\gamma} \mid \gamma^{\prime}=z_{(\beta, i)}^{\gamma^{\prime}}$.

F.3. para todos $\beta<\gamma^{\prime}<\gamma$ e $i \in\{0,1\}, L_{(\beta, i)}^{\gamma} \backslash L_{(\beta, i)}^{\gamma^{\prime}}$ e $R_{(\beta, i)}^{\gamma} \backslash R_{(\beta, i)}^{\gamma^{\prime}}$ são finitos.

Definidos $\left(K_{\gamma}\right)_{\gamma<\alpha}$ e $\left(P_{\gamma}\right)_{\gamma<\alpha}$ para $\alpha$ um ordinal limite, definimos

G.1. $K_{\alpha}$ é o limite inverso de $\left(K_{\gamma}\right)_{\gamma<\alpha}$;

G.2. Para todos $\beta<\alpha$ e $i \in\{0,1\}, z_{(\beta, i)}^{\alpha}=\bigcup_{\beta<\gamma<\alpha} z_{\beta}^{\gamma}$;

G.3. $L_{(\beta, i)}^{\alpha}$ é uma pseudointersecção infinita de $\left(L_{(\beta, i)}^{\gamma}\right)_{\beta<\gamma<\alpha}$, isto é, $L_{(\beta, i)}^{\alpha} \backslash$ $L_{(\beta, i)}^{\gamma}$ é finito, para todo $\gamma<\alpha$ (a existência dessa pseudointersecção está mostrada em [Do], Teorema 3.1.);

G.4. $R_{(\beta, i)}^{\alpha}$ é uma pseudointersecção infinita de $\left(R_{(\beta, i)}^{\gamma}\right)_{\beta<\gamma<\alpha}$.

Trabalhemos no caso sucessor. Suponha definidos $\left(K_{\gamma}\right)_{\gamma \leq \alpha}$ e $\left(P_{\gamma}\right)_{\gamma \leq \alpha}$ e definiremos $K_{\alpha+1}$ e $P_{\alpha+1}$.

Diremos que um passo $\alpha \in$ Par é não-trivial se:

H.1. $\left(x_{n}(\alpha)\right)_{n \in \omega}$ é uma seqüência relativamente discreta de pontos distintos de $K_{\alpha}$;

H.2. existem funções contínuas $g_{n}:[0,1]^{\alpha} \longrightarrow[0,1]$ tais que $f_{n}(\alpha)=g_{n} \circ \pi_{\alpha}$;

H.3. $\left(\left.g_{n}\right|_{K_{\alpha}}: n \in \omega\right)$ é duas a duas disjunta;

H.4. $x_{n}(\alpha) \notin \operatorname{supp}\left(g_{m}\right)$, para todos $n, m \in \omega$ e $g_{m}$ como no item H.2;

H.5. $\left|\int_{K_{\alpha}} g_{n} d \mu_{n}(\alpha)\right|>\varepsilon(\alpha)$, para todo $n \in \omega$.

Diremos que um passo $\alpha \in$ Impar é não-trivial se:

I.1. $A_{\alpha}, B_{\alpha} \subseteq K_{\alpha}$;

I.2. $U_{\alpha}=\pi_{\alpha}^{-1}\left[\pi_{\alpha}\left[U_{\alpha}\right]\right]$ e $V_{\alpha}=\pi_{\alpha}^{-1}\left[\pi_{\alpha}\left[V_{\alpha}\right]\right]$;

I.3. $\overline{\pi_{\alpha}\left[U_{\alpha}\right] \cap A_{\alpha}} \cap \overline{\pi_{\alpha}\left[V_{\alpha}\right] \cap B_{\alpha}} \neq \emptyset$; 
Se o passo $\alpha$ é trivial, tomamos $K_{\alpha+1}=K_{\alpha} \times\{0\}, L_{(\beta, i)}^{\alpha+1}=L_{(\beta, i)}^{\alpha}, R_{(\beta, i)}^{\alpha+1}=$ $R_{(\beta, i)}^{\alpha}, z_{(\beta, i)}^{\alpha+1}=z_{(\beta, i)}^{\alpha \subsetneq} 0, L_{(\alpha, i)}^{\alpha+1}=R_{(\alpha, i)}^{\alpha+1}=\emptyset$ e $z_{(\alpha, i)}^{\alpha+1}$ qualquer.

Suponhamos que estamos no caso não-trivial. Separaremos os casos $\alpha \in$ Par e $\alpha \in I m p a r$. Consideremos, primeiro, o caso $\alpha \in P$ Par.

Considere as funções $g_{n}:[0,1]^{\alpha} \longrightarrow[0,1]$ tais que $f_{n}(\alpha)=g_{n} \circ \pi_{\alpha}$. Considere $h_{n}=\left.g_{n}\right|_{K_{\alpha}}$.

Como $K_{\alpha}$ é compacto e métrico, toda seqüência possui uma subseqüência convergente. Tome $z \in K_{\alpha}$ e $N^{\prime} \subseteq \omega$ tais que

$$
\lim _{n \in N^{\prime}} x_{n}(\alpha)=z \text {. }
$$

Como $x_{n}(\alpha)$ são pontos distintos, tirando, eventualmente, um elemento de $N^{\prime}$, podemos assumir que $x_{n}(\alpha) \neq z$, para todo $n \in N^{\prime}$, e, portanto, para todo $\alpha^{\prime} \geq \alpha$ temos

$$
\pi_{K_{\alpha^{\prime}}, K_{\alpha}}^{-1}(\{z\}) \cap F_{n}^{\alpha}\left(\alpha^{\prime}\right)=\emptyset .
$$

Pelos Lema 1.23 existem $a \subseteq N^{\prime}$ infinito, $h_{n}^{\prime}: K_{\alpha} \longrightarrow[0,1]$ contínuas, para $n \in a$, e $\delta>0$ tais que

J.1. $\operatorname{supp}\left(h_{n}^{\prime}\right) \subseteq \operatorname{supp}\left(h_{n}\right)$, para todo $n \in a$;

J.2. Para todo $b \subseteq a$, a extensão de $K_{\alpha}$ por $\left(h_{n}^{\prime}\right)_{n \in b}$ é forte;

J.3. Para todo $n \in a,\left|\int h_{n}^{\prime} d \mu_{n}(\alpha)\right|>\delta$ e $\Sigma\left\{\int h_{n}^{\prime} d\left|\mu_{n}(\alpha)\right|: m \neq n, m \in\right.$ $a\}<\frac{\delta}{3}$

J.4. $\Delta\left(\left(h_{n}^{\prime}\right)_{n \in a}\right)$ é unitário ou disjunto de $\left\{z_{(\beta, i)}^{\alpha}:(\beta, i) \in \alpha \times\{0,1\}\right\} \cup$ $\left\{x_{n}(\alpha): n \in \omega\right\}$.

Observe que, pelo Lema 1.16, se $L$ é uma extensão de $K_{\alpha}$ por $\left(h_{n}^{\prime}\right)_{n \in b}$, para algum $b \subseteq a$, então $\left|\pi_{L, K}^{-1}(x)\right|=1$, para todo $x \notin \Delta\left(\left(h_{n}^{\prime}\right)_{n \in a}\right)$. Iremos prosseguir a construção separando em dois casos:

Caso $1 \Delta\left(\left(h_{n}^{\prime}\right)_{n \in a}\right)$ é disjunto de $\left\{z_{(\beta, i)}^{\alpha}:(\beta, i) \in \alpha \times\{0,1\}\right\} \cup\left\{x_{n}(\alpha): n \in\right.$ $\omega\}$.

Neste caso tomamos qualquer $b \subseteq a$ infinito e co-infinito em $a$, e tomamos $K_{\alpha+1}$ a extensão de $K_{\alpha}$ por $\left(h_{n}^{\prime}\right)_{n \in b}, L_{(\alpha, i)}^{\alpha+1}=b, R_{(\alpha, i)}^{\alpha+1}=a \backslash b, z_{(\alpha, i)}^{\alpha+1}=z$, para $(\beta, i) \in \alpha \times\{0,1\}, L_{(\beta, i)}^{\alpha+1}=L_{(\beta, i)}^{\alpha}, R_{(\beta, i)}^{\alpha+1}=R_{(\beta, i)}^{\alpha}$ e $z_{(\beta, i)}^{\alpha+1}$ o único elemento de $\pi^{-1}\left(\left\{z_{(\beta, i)}^{\alpha}\right\}\right)$. 
Observe que, como $F_{n}^{\beta}(\alpha)$ converge para $z_{(\beta, i)}^{\alpha}$, para $n \in L_{(\beta, i)}^{\alpha} \cup R_{(\beta, i)}^{\alpha}$, e, numa vizinhança de $z_{(\beta, i)}^{\alpha}, K_{\alpha+1}$ é o gráfico de uma função contínua, temos que $F_{n}^{\beta}(\alpha+1)$ converge para $z_{(\beta, i)}^{\alpha+1}$, em $K_{\alpha+1}$, para $n \in L_{(\beta, i)}^{\alpha+1} \cup R_{(\beta, i)}^{\alpha+1}$.

Caso $2 \Delta\left(\left(h_{n}^{\prime}\right)_{n \in a}\right)$ é unitário.

Seja $y$ esse único ponto que é bifurcado numa extensão de $K_{\alpha}$ por $\left(h_{n}^{\prime}\right)_{n \in a}$. Isso significa que $\operatorname{supp}\left(h_{n}^{\prime}\right) \stackrel{n \in a}{\longrightarrow} y$, pois, se isso não ocorresse, teríamos uma vizinhança $V$ de $y$ e um $c \subseteq a$ infinito tais que para todo $n \in a$ existiria $y_{n} \in \operatorname{supp}\left(h_{n}^{\prime}\right) \backslash V$. Tomando $y^{\prime}$ um ponto de acumulação de $\left\{y_{n}: n \in c\right\}$ teríamos $y^{\prime} \in \Delta\left(\left(h_{n}^{\prime}\right)_{n \in a}\right)$ e $y^{\prime} \neq y$, contradizendo que $\Delta\left(\left(h_{n}^{\prime}\right)_{n \in a}\right)$ é unitário.

Fixamos $i \in\{0,1\}$. Sejam $\left(\beta_{n}\right)_{n \in \omega}$ os ordinais tais que $z_{\left(\beta_{n}, i\right)}^{\alpha}=y$. Nos outros ordinais procedemos como no caso 1 na construção de $L_{(\beta, i)}^{\alpha+1}, R_{(\beta, i)}^{\alpha+1}$ e $z_{(\beta, i)}^{\alpha+1}$.

Sejam $b \subseteq a, L_{(\beta, i)}^{\alpha+1} \subseteq L_{(\beta, i)}^{\alpha}$ e $R_{(\beta, i)}^{\alpha+1} \subseteq R_{(\beta, i)}^{\alpha}$ infinitos tais que

(**) $\quad F_{n}^{\beta_{m}}(\alpha) \cap \operatorname{supp}\left(h_{k}^{\prime}\right)=\emptyset, \forall \beta \leq \alpha, m \in \omega, k \in b, n \in L_{(\beta, i)}^{\alpha+1} \cup R_{(\beta, i)}^{\alpha+1}$.

Para $\beta=\alpha$, a existência de tais conjuntos segue da hipótese, $x_{n}(\alpha) \notin$ $\operatorname{supp}\left(h_{m}^{\prime}\right)$. Para $\beta<\alpha$ usamos a seguinte afirmação.

Afirmação 2.1.1. Sejam $F_{n, m}$ fechados tais que, para cada $m \in \omega, F_{n, m} \stackrel{n}{\longrightarrow}$ y e sejam $G_{n}$ fechados tais que $G_{n} \longrightarrow y$, com $y \notin G_{n}$ e $y \notin F_{n, m}$, para todos $n, m$. Então existem subconjuntos infinitos $b \subseteq \omega$ e $c_{m} \subseteq \omega$, para $m \in \omega$, tais que

$$
F_{n, m} \cap G_{k}=\emptyset, \forall n \in c_{m}, m \in \omega, k \in b .
$$

Para demonstrar a afirmação, construiremos $\left(U_{n}\right)_{n \in \omega}$ e $\left(V_{n}\right)_{n \in \omega}$, vizinhanças abertas de $y$, tais que $U_{n+1} \subseteq V_{n} \subseteq U_{n}$, juntamente com inteiros crescentes $\left(k_{n}\right)_{n \in \omega}$ e $\left(l_{n}\right)_{n \in \omega}$.

Tomamos $U_{0}$ qualquer. Definidos $U_{n},\left(k_{j}\right)_{j<n}$ e $\left(l_{j}\right)_{j<n}$, tomamos $k_{n}>k_{j}$, para todo $j<n$, tal que $F_{k_{n}, m} \subseteq U_{n}$, para todo $m \leq n$. Seja $V_{n} \subseteq U_{n}$ uma vizinhança de $y$ disjunta de $F_{k_{j}, m}$, para todos $j \leq n$ e $m \leq j$. Tome $l_{n}>l_{j}$, para todo $j<n$, tal que $G_{l_{n}} \subseteq V_{n}$. Seja $U_{n+1}$ vizinhança aberta de $y$ disjunta de $G_{l_{n}}$.

Defina $b=\left\{l_{n}: n \in \omega\right\}$ e $c_{m}=\left\{k_{n}: n \geq m\right\}$. Sejam $m, j \in \omega$ e $n \geq m$. Temos $F_{k_{n}, m} \subseteq U_{n} \backslash V_{n}$ e $G_{l_{j}} \subseteq V_{j} \backslash U_{j+1}$. Se $n \leq j$ temos $F_{k_{n}, m} \cap V_{n}=\emptyset \mathrm{e}$ $G_{l_{j}} \subseteq V_{j} \subseteq V_{n}$. Se $n>j$ temos $F_{k_{n}, m} \subseteq U_{n} \subseteq U_{j}$ e $G_{l_{j}} \cap U_{j}=\emptyset$. Em ambos casos temos $F_{k_{n}, m} \cap G_{l_{j}}=\emptyset$ provando a afirmação. 
Por $(*)$ sabemos que as hipótese $y \notin F_{n, m}$ da afirmação é satisfeita para $F_{n, m}=F_{n}^{\beta_{m}}(\alpha)$ e $G_{n}=\operatorname{supp}\left(h_{n}^{\prime}\right)$.

Tome $L_{(\alpha, i)}^{\alpha+1}=b$, para $i \in\{0,1\}$ como acima, mas de modo a satisfazer $b \subseteq a$ e $a \backslash b$ infinito. Tome $R_{(\alpha, i)}^{\alpha+1}=a \backslash b$. Defina $K_{\alpha+1}$ a extensão de $K_{\alpha}$ por $\left(h_{n}^{\prime}\right)_{n \in b}$. Tome $z_{(\beta, i)}^{\alpha+1}=z_{(\beta, i)}^{\alpha \frown} 0$, nos casos em que $z_{(\beta, i)}^{\alpha}=y$, e $z_{(\beta, i)}^{\alpha+1}$ o único elemento de $\pi^{-1}\left(z_{(\beta, i)}^{\alpha}\right)$, nos outros casos.

Vejamos que $F_{n}^{\beta}(\alpha+1) \longrightarrow z_{(\beta, i)}^{\alpha+1}$, para $n \in L_{(\beta, i)}^{\alpha+1}$ e $n \in R_{(\beta, i)}^{\alpha+1}$. Nos casos em que $z_{(\beta, i)}^{\alpha}=y$, por $(* *)$ temos $F_{n}^{\beta}(\alpha+1)=F_{n}^{\beta}(\alpha) \times\{0\}$, que converge para $z_{(\beta, i)}^{\alpha \subsetneq 0} 0=z_{(\beta, i)}^{\alpha+1}$. Nos outros casos, é como no caso 1 , isto é, $F_{n}^{\beta}(\alpha+1)$ é uma imagem homeomórfica de $F_{n}^{\beta}(\alpha)$, numa vizinhança de $z_{(\beta, i)}^{\alpha+1}$.

Seja $\alpha \in$ Impar um caso não-trivial. Seja $z=\lim _{n \rightarrow \infty} x_{n}(\alpha)$ um elemento de $K_{\alpha}$. Usando o Lema de Urysohn podemos definir uma seqüência de funções duas a duas disjuntas $\left(h_{n}\right)_{n \in 2 \omega}$ tal que $\Delta\left(\left(h_{n}\right)_{n \in 2 \omega}\right)=\{z\}$ e, para todo $n \in 2 \omega$ e $m \in \omega$,

$$
h_{n}\left(x_{m}(\alpha)\right)= \begin{cases}1, & \text { se } m=n \text { ou } m=n+1 \\ 0, & \text { caso contrário }\end{cases}
$$

Seja $b \subseteq 2 \omega$ tal que $K_{\alpha}\left(\left(h_{n}\right)_{n \in b}\right)$ é uma extensão forte. Defina $K_{\alpha+1}=$ $K_{\alpha}\left(\left(h_{n}\right)_{n \in b}\right), L_{(\alpha, 1)}^{\alpha+1}=b, L_{(\alpha, 0)}^{\alpha+1}=2 \omega \backslash b, R_{(\alpha, 1)}^{\alpha+1}=\left\{n+1: n \in L_{(\alpha, 1)}^{\alpha+1}\right\}$, $R_{(\alpha, 0)}^{\alpha+1}=\left\{n+1: n \in L_{(\alpha, 0)}^{\alpha+1}\right\}, z_{(\alpha, 0)}^{\alpha+1}=z \frown 0$ e $z_{(\alpha, 1)}^{\alpha+1}=z \frown 1$

Notemos que $F_{n}^{\alpha}(\alpha+1)=\left\{x_{n}(\alpha) \frown 1\right\}$, se $n \in L_{(\alpha, 1)}^{\alpha+1} \cup R_{(\alpha, 1)}^{\alpha+1}$, e $F_{n}^{\alpha}(\alpha+1)=$ $\left\{x_{n}(\alpha) \frown 0\right\}$, caso contrário. Portanto $F_{n}^{\alpha}(\alpha+1) \rightarrow z_{(\alpha, 0)}^{\alpha+1}$, para $n \in L_{(\alpha, 0)}^{\alpha+1} \cup$ $R_{(\alpha, 0)}^{\alpha+1}$, e $F_{n}^{\alpha}(\alpha+1) \rightarrow z_{(\alpha, 1)}^{\alpha+1}$, para $n \in L_{(\alpha, 1)}^{\alpha+1} \cup R_{(\alpha, 1)}^{\alpha+1}$.

No restante da construção de $P_{\alpha+1}$, ou seja, para definir $L_{(\beta, i)}^{\alpha+1}, R_{(\beta, i)}^{\alpha+1}$ e $z_{(\beta, i)}^{\alpha+1}$, para $\beta<\alpha$, agimos como no caso 2 de um passo $\alpha \in$ Par não-trivial.

Feita a construção, tome $K$ o limite inverso de $\left(K_{\alpha}\right)_{\alpha<\omega_{1}}$. Sejam $\left(f_{n}\right.$ : $n \in \omega),\left(x_{n}: n \in \omega\right),\left(\mu_{n}: n \in \omega\right)$ e $\varepsilon$ como na hipótese do teorema. Podemos assumir, sem perda de generalidade, que $x_{n} \notin \operatorname{supp}\left(f_{m}\right)$, para todos $n, m \in \omega$. Para isso basta usarmos o Lema de Urysohn e a regularidade de $\mu_{n}$ para reduzir os suportes de $f_{n}$ preservando condição $d$ ) da hipótese.

Pelo Teorema de Tietze estendemos $f_{n}$ para $\tilde{f}_{n}:[0,1]^{\omega_{1}} \longrightarrow[0,1]$. Pelo Teorema de Mibu (vide [Mi]) existem $\alpha<\omega_{1}$ e funções $g_{n}:[0,1]^{\alpha} \longrightarrow[0,1]$ tais que $\tilde{f}_{n}=g_{n} \circ \pi$. Notemos que $f_{n}=\left.g_{n}\right|_{K_{\alpha}} \circ \pi_{\alpha}$. Como a existência de tais funções ainda valem para um $\alpha^{\prime}>\alpha$, uma vez que $\tilde{f}_{n} \circ \pi_{\alpha^{\prime}}=g_{n} \circ \pi_{\alpha} \circ \pi_{\alpha^{\prime}}$, podemos tomar $\alpha \in$ Par um passo não-trivial da construção tal que: 
K.1. $f_{n}(\alpha)=\tilde{f}_{n}$, para todo $n \in \omega$;

K.2. $x_{n}(\alpha)=x_{n} \mid \alpha$, para todo $n \in \omega$;

K.3. $\varepsilon(\alpha)=\varepsilon$;

K.4. $\mu_{n}(\alpha)=\mu_{n} \mid \mathcal{B}_{\alpha}$, para todo $n \in \omega$.

Tomamos $b=L_{(\alpha, 0)}^{\alpha+1}$ e $a=L_{(\alpha, 0)}^{\alpha+1} \cup R_{(\alpha, 0)}^{\alpha+1}$ e $f_{n}^{\prime}=h_{n}^{\prime} \circ \pi$, onde $h_{n}^{\prime}$ são as funções construídas no passo sucessor na construção de $K$, ou seja $K_{\alpha+1}=$ $K_{\alpha}\left(\left(h_{n}^{\prime}\right)_{n \in a}\right)$. Tomamos $\delta>0$ que obtemos na construção de $h_{n}^{\prime}$, isto é, satisfazendo J.3.

Pelo Lema 1.17, $\left(h_{n}^{\prime} \circ \pi\right)_{n \in b}$ tem supremo em $C\left(K_{\alpha+1}\right)$, pois esta é uma extensão forte por essas funções. Do Lema 1.20 segue que $\left(f_{n}^{\prime}\right)_{n \in b}$ tem supremo em $C(K)$.

Note que $\int f_{n}^{\prime} d \mu_{m}=\int h_{n}^{\prime} d \mu_{m}(\alpha)$, para todos $n, m$, pois $f_{n}^{\prime}$ é determinada pelas coordenadas abaixo de $\alpha$. Portanto, da construção segue também a propriedade $e$ ).

A conexidade de $K$ segue do Lema 1.20 .

Falta mostrar a propriedade $g$ ). Suponha que existam abertos $U_{1}$ e $U_{2}$ de $K$ tais que $x_{n}(\alpha) \in U_{1}$, para todo $n \in b$, e $x_{n}(\alpha) \in U_{2}$, para todo $n \in a \backslash b$. Pela compacidade, podemos assumir que $U_{1}$ e $U_{2}$ são uniões finitas de abertos elementares. Portanto existe $\beta<\omega_{1}$, podendo assumir $\beta>\alpha$, tal que a separação ocorre em $\beta$, isto é, $\overline{\left\{x_{n} \mid \beta: n \in b\right\}} \cap \overline{\left\{x_{n} \mid \beta: n \in a \backslash b\right\}}=\emptyset$, em $K_{\beta}$. Como $x_{n} \mid \alpha=x_{n}(\alpha)$, temos que $x_{n} \mid \beta \in F_{n}^{\alpha}(\beta)$. Mas, pela construção, $L_{(\alpha, 0)}^{\beta} \backslash$ $L_{(\alpha, 0)}^{\alpha+1}$ é finito. Como $L_{(\alpha, 0)}^{\alpha+1}=b$, e $\lim _{n \in L_{(\alpha, 0)}^{\beta}} F_{n}^{\alpha}(\beta)=z_{(\alpha, 0)}^{\beta}$, temos que $z_{(\alpha, 0)}^{\beta} \in$ $\overline{\left\{x_{n} \mid \beta: n \in b\right\}}$. Da mesma forma concluímos que $z_{(\alpha, 0)}^{\beta} \in \overline{\left\{x_{n} \mid \beta: n \in a \backslash b\right\}}$, contradizendo que $\overline{\left\{x_{n} \mid \beta: n \in b\right\}} \cap \overline{\left\{x_{n} \mid \beta: n \in a \backslash b\right\}}=\emptyset$.

Mostraremos a parte $i$ i) do teorema. Sejam $L \subseteq K$ fechado e $V_{1}$ e $V_{2}$ abertos disjuntos de $L$ tais que $\bar{V}_{1} \cap \bar{V}_{2} \neq \emptyset$. Sejam $U$ e $V$ abertos de $[0,1]^{\omega_{1}}$ tais que $V_{1}=U \cap L$ e $V_{2}=V \cap L$. Como $L$ é fechado, $\bar{V}_{1} \cap \bar{V}_{2}=\bar{U} \cap \bar{V} \cap L$, pois $\overline{U \cap L}=\bar{U} \cap L$.

Como $[0,1]^{\omega_{1}}$ é separável (vide $\left.[E n g], 2.3 .16\right)$ então $[0,1]^{\omega_{1}}$ é c.c.c., isto é, não contém uma seqüência não-enumerável de abertos disjuntos. Portanto podemos assumir que $U$ e $V$ são uniões enumeráveis de abertos elementares. De fato, se tomarmos $U^{\prime} \subseteq U$ a união de uma família maximal de abertos elementares contidos em $\bar{U}$, temos $\overline{U^{\prime}}=\bar{U}$, o mesmo podendo ser feito para $V$. 
Sejam $\left(y_{\alpha}\right)_{\alpha<\omega_{1}}$ e $\left(z_{\alpha}\right)_{\alpha<\omega_{1}}$ seqüências que formam conjuntos densos em $V_{1}$ e $V_{2}$, respectivamente. Tome $\beta<\omega_{1}$ que contém as coordenadas que determinam $U$ e $V$, isto é, $\pi^{-1}\left[\pi_{\beta}[U]\right]=U$ e $\pi^{-1}\left[\pi_{\beta}[V]\right]=V$. Usando o Lema 1.28, escolha $\alpha>\beta$ tal que $\alpha \in \operatorname{Impar}, U_{\alpha}=U, V_{\alpha}=V,\left(y_{\beta} \mid \alpha\right)_{\beta<\alpha}$ é denso em $\pi_{\alpha}\left[V_{1}\right],\left(z_{\beta} \mid \alpha\right)_{\beta<\alpha}$ é denso em $\pi_{\alpha}\left[V_{2}\right]$ e

$$
\begin{aligned}
& \left\{y_{\beta} \mid \alpha: \beta<\alpha\right\}=A_{\alpha} \\
& \left\{z_{\beta} \mid \alpha: \beta<\alpha\right\}=B_{\alpha} .
\end{aligned}
$$

Seja $x \in \bar{V}_{1} \cap \bar{V}_{2}$. Como $A_{\alpha}$ e $B_{\alpha}$ são densos em $\pi_{\alpha}\left[V_{1}\right]$ e $\pi_{\alpha}\left[V_{2}\right]$, respectivamente, então $x \mid \alpha \in \overline{\pi_{\alpha}\left[U_{\alpha}\right] \cap A_{\alpha}} \cap \overline{\pi_{\alpha}\left[V_{\alpha}\right] \cap B_{\alpha}}$. Estamos, portanto, em um passo $\alpha \in$ Impar não-trivial. Temos então $x_{n}(\alpha) \stackrel{n \in \omega}{\longrightarrow} x \mid \alpha$ e $x_{n}(\alpha) \in \pi_{\alpha}\left[V_{1}\right]$, para $n$ par, e $x_{n}(\alpha) \in \pi_{\alpha}\left[V_{2}\right]$, para $n$ ímpar.

Para cada $n$ par, seja $\alpha_{n}$ tal que $y_{\alpha_{n}} \mid \alpha=x_{n}(\alpha)$. Para cada $n$ ímpar, seja $\alpha_{n}$ tal que $z_{\alpha_{n}} \mid \alpha=x_{n}(\alpha)$. Analogamente à demonstração do item $\left.g\right)$ da parte $i$ ) do teorema, mostramos que

$$
\begin{aligned}
& \overline{\left\{y_{\alpha_{n}}: n \in L_{(\alpha, 0)}^{\alpha+1}\right\}} \cap \overline{\left\{z_{\alpha_{n}}: n \in R_{(\alpha, 0)}^{\alpha+1}\right\}} \neq \emptyset ; \\
& \overline{\left\{y_{\alpha_{n}}: n \in L_{(\alpha, 1)}^{\alpha+1}\right\}} \cap \overline{\left\{z_{\alpha_{n}}: n \in R_{(\alpha, 1)}^{\alpha+1}\right\}} \neq \emptyset .
\end{aligned}
$$

Tome $z_{1} \in \overline{\left\{y_{\alpha_{n}}: n \in L_{(\alpha, 0)}^{\alpha+1}\right\}} \cap \overline{\left\{z_{\alpha_{n}}: n \in R_{(\alpha, 0)}^{\alpha+1}\right\}}$ e $z_{2} \in \overline{\left\{y_{\alpha_{n}}: n \in L_{(\alpha, 1)}^{\alpha+1}\right\}} \cap$ $\overline{\left\{z_{\alpha_{n}}: n \in R_{(\alpha, 1)}^{\alpha+1}\right\}}$. Temos $z_{1}, z_{2} \in \bar{V}_{1} \cap \bar{V}_{2} \mathrm{e}$

$$
z_{1}\left|(\alpha+1)=z_{(\alpha, 0)}^{\alpha+1} \neq z_{(\alpha, 1)}^{\alpha+1}=z_{2}\right|(\alpha+1) .
$$

Logo $\left|\bar{V}_{1} \cap \bar{V}_{2}\right| \geq 2$, como queríamos mostrar.

Teorema 2.2. Assuma $\diamond$. Existe um espaço compacto e conexo $K$ tal que, para todo $L \subseteq K$ fechado, todo operador em $C(L)$ é da forma $g I+S$, para algum $g \in C(L)$ e $S$ fracamente compacto. Em particular, se $L \subseteq K$ é conexo então $C(L)$ é indecomponível.

Demonstração: Tome $K$ como no Teorema 2.1. Seja $L \subseteq K$ fechado. Seja $T: C(L) \longrightarrow C(L)$ um operador contínuo. Mostraremos que $T$ é um multiplicador fraco. Essa demonstração será uma adaptação do Lema 5.2 de $[\mathrm{Ko} 2]$. 
Suponha que $T$ não seja um multiplicador fraco, isto é, existem uma seqüência duas a duas disjunta de elementos $e_{n} \in C(L)$ com imagens em $[-1,1], \varepsilon>0$ e pontos $x_{n} \in L$ tais que $e_{n}\left(x_{n}\right)=0$ para todo $n \in \omega$ e $\left|T\left(e_{n}\right)\left(x_{n}\right)\right|>\varepsilon$ para infinitos $n$ 's. Assumimos, então, que vale para todo $n$.

Como somas finitas de $e_{n}$ 's são uniformemente limitadas, se $x_{n}$ fosse constante para infinitos $n$ 's, teríamos contradição com o fato de $T$ ser limitada (isto é, contínua). Portanto, podemos assumir que $x_{n}$ 's são todos distintos.

Podemos supor, sem perda de generalidade, que $e_{m}\left(x_{n}\right)=0$, para todos $n, m \in \omega:$ se existe um $k_{0}$ tal que $e_{k_{0}}\left(x_{n}\right) \neq 0$ para $n$ 's pertencentes a um conjunto infinito $N^{\prime} \subseteq \omega$, refinamos a seqüência para $N^{\prime} \backslash\left\{k_{0}\right\}$ e usamos que $e_{n}$ 's são disjuntas. Caso contrário, construímos por indução uma seqüência como queremos.

Considerando $\max \left(e_{n}, 0\right)-\min \left(e_{n}, 0\right)$ no lugar de $e_{n}$, podemos assumir que $e_{n}$ tem imagem em $[0,1]$.

Sejam $\mu_{n}=T^{*}\left(\delta_{x_{n}}\right)$, isto é, $\mu_{n}$ 's são medidas dadas pela relação

$$
T(f)\left(x_{n}\right)=\int f d \mu_{n}
$$

para todo $f \in C(L)$. Temos $\left|\int e_{n} d \mu_{n}\right|>\varepsilon$, para todo $n$. Note que $\left(\mu_{n}\right)_{n \in \omega}$ é uma seqüência limitada de medidas. Usando o Lema de Rosenthal achamos $N^{\prime} \subseteq \omega$ infinito tal que

$$
\Sigma\left\{\left|\int e_{m} d \mu_{n}\right|: n \neq m, m \in N^{\prime}\right\}<\varepsilon / 3 .
$$

Agora estendemos continuamente as funções $e_{n}$ 's para funções $f_{n} \in C(K)$, também disjuntas e com imagens em $[0,1]$. Para isso usamos o Teorema de Tietze para estender $e_{n}$ e, usando o Lema de Urysohn, multiplicamos por uma função que vale 1 em $\operatorname{supp}\left(e_{n}\right)$ (que também é fechado em $K$ ) e 0 em $\cup\left\{\operatorname{supp}\left(f_{k}\right): k<n\right\}$.

Como $L \subseteq K$, interpretamos $\mu_{n}$ como medidas medidas sobre $K$, isto é, $\mu_{n}(A)=\mu_{n}^{\prime}(A \cap L)$, para todo boreliano $A \subseteq K$. Assim, para todos $n, m \in \omega$,

$$
\int_{K} f_{m} d \mu_{n}=\int_{L} e_{m} d \mu_{n} .
$$

Aplicando o Teorema 2.1 para $\left\{f_{n}: n \in N^{\prime}\right\},\left\{x_{n}: n \in N^{\prime}\right\},\left\{\mu_{n}\right.$ : $\left.n \in N^{\prime}\right\}$ e $\varepsilon$, achamos $b \subseteq a \subseteq N^{\prime}, \delta>0$ e funções $\left\{f_{n}^{\prime}: n \in a\right\}$ como no enunciado do teorema. 
Podemos assumir que

$$
\int \sup \left\{f_{m}^{\prime}: m \in b\right\} d \mu_{n}=\int \Sigma_{m \in b} f_{m}^{\prime} d \mu_{n},
$$

para todo $n \in \omega$. Para isso, tome $\left(N_{\xi}\right)_{\xi<\omega_{1}}$ uma família de subconjuntos infinitos de $N^{\prime}$ tal que $N_{\xi} \cap N_{\eta}$ é finito, para todos $\xi \neq \eta$ (por exemplo, identificando $\omega \operatorname{com} \mathbb{Q}$ e $\omega_{1}$ com $\mathbb{R}$, tome $N_{\xi}$ uma seqüência de racionais que converge para $\xi$ ). Para cada $\xi$ tome $b_{\xi} \subseteq a_{\xi} \subseteq N_{\xi}$ como $a$ e $b$ do Teorema 2.1.

Para cada $\xi<\omega_{1}$ e $n \in b_{\xi}$ tome $f_{n}^{\xi}$ como $f_{n}^{\prime}$ no teorema, isto é, as propriedades $e), f$ ) e $g$ ) do Teorema 2.1 são satisfeitas para $f_{n}^{\prime}=f_{n}^{\xi}, a=a_{\xi}$ e $b=b_{\xi}$.

Afirmação 2.2.1. Existe $\xi<\omega_{1}$ tal que

$$
\int\left[\sup \left\{f_{m}^{\xi}: m \in b_{\xi}\right\}-\Sigma_{m \in b_{\xi}} f_{m}^{\xi}\right] d \mu_{n}=0,
$$

para todo $n \in \omega$.

Para cada $\xi<\omega_{1}$ e $c \subseteq b_{\xi}$ definimos $f_{c}^{\xi}=\sup \left\{f_{m}^{\xi}: m \in c\right\}-\Sigma_{m \in c} f_{m}^{\xi}$ quando esse supremo existe. Note que, se $F \subseteq b_{\xi}$ é finito, temos

$$
\sup \left\{f_{m}^{\xi}: m \in b_{\xi}\right\}=\sup \left\{f_{m}^{\xi}: m \in b_{\xi} \backslash F\right\}+\Sigma_{m \in F} f_{m}^{\xi}
$$

e, portanto, $f_{b_{\xi} \backslash F}^{\xi}=f_{b_{\xi}}^{\xi}$. Em particular $f_{b_{\xi}}^{\xi}=f_{b_{\xi} \backslash b_{\xi^{\prime}}}^{\xi}$, para todos $\xi, \xi^{\prime}<\omega_{1}$ distintos, já que $b_{\xi} \cap b_{\xi^{\prime}}$ é finito.

Fixe $\xi \neq \xi^{\prime}$. Sejam $g=\sup \left\{f_{n}^{\xi}: n \in b_{\xi} \backslash b_{\xi^{\prime}}\right\}$ e $h=\sup \left\{f_{n}^{\xi^{\prime}}: n \in b_{\xi^{\prime}} \backslash b_{\xi}\right\}$. Como $\operatorname{supp}\left(f_{n}^{\eta}\right) \subseteq \operatorname{supp}\left(f_{n}\right)$, para todo $n \in \omega$ e todo $\eta<\omega_{1}$, temos que $f_{n}^{\xi} \cdot f_{m}^{\xi^{\prime}}=0$, para todos $n \neq m$. Vejamos agora que $g \cdot h=0$.

Suponha que exista $x \in K$ tal que $g(x)>0$ e $h(x)>0$. Então existe uma vizinhança $V$ de $x$ tal que $g$ e $h$ são positivas em todos os pontos de $V$. Então existem $y \in V$ e $n \in b_{\xi} \backslash b_{\xi^{\prime}}$ tais que $f_{n}^{\xi}(y)>0$. Como $f_{n}^{\xi} \cdot f_{m}^{\xi^{\prime}}=0$, para todo $m \in b_{\xi^{\prime}} \backslash b_{\xi}$, se tomarmos $\varphi: K \longrightarrow[0,1]$ contínua tal que $\varphi(y)=1 \mathrm{e}$ $\varphi$ é nula nos pontos em que $f_{n}^{\xi}$ é nula, temos que $f_{m}^{\xi^{\prime}} \leq h \cdot \varphi<h$, para todo $m \in b_{\xi^{\prime}} \backslash b_{\xi}$, contradizendo a definição de $h$.

Como $f_{b_{\xi}}^{\xi}=f_{b_{\xi} \backslash b_{\xi^{\prime}}}^{\xi} \leq g$ e $f_{b_{\xi^{\prime}}}^{\xi^{\prime}}=f_{b_{\xi^{\prime}} \backslash b_{\xi}}^{\xi^{\prime}} \leq h$, segue que $f_{b_{\xi}}^{\xi} \cdot f_{b_{\xi^{\prime}}}^{\xi^{\prime}}=0$, para todos $\xi \neq \xi^{\prime}$. Logo existe $\xi<\omega_{1}$ tal que $f_{b_{\xi}}^{\xi}$ tem integral nula com respeito a todas as medidas $\mu_{n}$ 's, concluindo a demonstração da afirmação. 
Tomando $f=\sup \left\{f_{n}^{\prime}: n \in b\right\}$ e $n \in b$ temos

$$
\begin{aligned}
\left|T\left(\left.f\right|_{L}\right)\left(x_{n}\right)\right|=\left|\int_{K} f d \mu_{n}\right| & =\left|\int f_{n}^{\prime} d \mu_{n}+\int \Sigma\left\{f_{m}^{\prime}: m \neq n, m \in b\right\} d \mu_{n}\right| \\
& \geq \delta-\delta / 3=2 \delta / 3 .
\end{aligned}
$$

Por outro lado, se $n \in a \backslash b$ temos

$$
\left|T\left(\left.f\right|_{L}\right)\left(x_{n}\right)\right|=\left|\int_{K} \Sigma_{m \in b} f_{m}^{\prime} d \mu_{n}\right| \leq \delta / 3 .
$$

Como $T\left(\left.f\right|_{L}\right)$ é contínuo, temos que os fechos de $\left\{x_{n}: n \in b\right\}$ e $\left\{x_{n}: n \in\right.$ $a \backslash b\}$ são disjuntos, contradizendo o item $g$ ) do Teorema 2.1.

O restante do teorema segue do Teorema 2.1 item $i i$ ), dos Lemas 1.10, 1.12 e do Teorema 1.11.

Corolário 2.3. O espaço $C(K)$ como acima tem pelo menos contínuo quocientes indecomponíveis da forma $C(L)$ não isomorfos.

Demonstração: Primeiro vejamos que, se $C(L)$ tem poucos operadores, então não é isomorfo a nenhum de seus quocientes próprios. De fato, seja $X$ um quociente próprio de $C(L)$, e seja $T: C(L) \longrightarrow X$ uma transformação linear sobrejetora. Suponha que existe $S: X \longrightarrow C(L)$ um isomorfismo. Como $C(L)$ tem poucos operadores, $S \circ T$ é multiplicador fraco e, pelo Lema 1.8, $S \circ T$ é sobrejetor se, e somente se, é um isomorfismo sobre a imagem. Mas $S$ e $T$ são ambos sobrejetores, de onde concluímos que $S \circ T$ é um isomorfismo de $C(K)$. Logo $T$ precisa ser injetora e, portanto, $X$ é isomorfo a $C(K)$.

Para cada $r \in[0,1]$, tome $K_{r}=\pi_{K,[0,1]^{2}}^{-1}\left([0, r]^{2}\right)$. Pelo Lema 1.18 podemos concluir que cada $K_{r}$ é conexo. Se $r<s$ temos $K_{r} \subset K_{s}$ e, portanto, $C\left(K_{r}\right)$ e $C\left(K_{s}\right)$ são indecomponíveis e não são isomorfos.

\subsection{Um espaço $C(K)$ indecomponível tal que $K$ contém $\beta \mathbb{N}$}

O Teorema 2.2 mostra que, assumindo $\diamond$, existe um compacto $K$ tal que, para todo $L \subseteq K, C(L)$ tem poucos operadores. Em particular, $K$ não 
contém $\beta \mathbb{N}$. Nesta seção, assumindo $\mathrm{CH}$, construímos um espaço compacto $K$ conexo, contendo $\beta \mathbb{N}$, tal que todo operador em $C(K)$ é da forma $g I+S$, onde $g \in C(K)$ e $S$ é um operador fracamente compacto. Não sabemos se $\mathrm{CH}$ é necessário para obtermos o Teorema 2.5.

Lema 2.4. Seja K um compacto Hausdorff. São equivalentes:

i) $K$ contém um subespaço homeomorfo a $\beta \mathbb{N}$;

ii) Existe uma seqüência $\left\{x_{n}: n \in \omega\right\} \subseteq K$ relativamente discreta tal que, para todo $a \subseteq \omega, \overline{\left\{x_{n}: n \in a\right\}} \cap \overline{\left\{x_{n}: n \in \omega \backslash a\right\}}=\emptyset$.

Demonstração: $i$ ) implica $i i)$ é trivial, tomando $x_{n}$ o elemento de $K$ correspondente a $n^{*}$. Vejamos que $\left.i i\right)$ implica $i$ ). Seja $\left\{x_{n}: n \in \omega\right\}$ como em $i i$ ) e tome $X=\overline{\left\{x_{n}: n \in \omega\right\}}$. Veremos que $X$ é homeomorfo a $\beta \mathbb{N}$. Como $X$ é fechado em $K$, para todo $E \subseteq X$ o fecho de $E$ em $X$ coincide com o fecho de $E$ em $K$. Usaremos a notação $\bar{E}$ para o fecho em $X$ e em $K$. Mostraremos as seguinte afirmações:

Afirmação 2.4.1. $\left\{\overline{\left\{x_{n}: n \in a\right\}}: a \subseteq \omega\right\}$ é uma base de abertos-fechados de $X$.

Sejam $x \in X$ e $V$ uma vizinhança aberta de $x$. Pela normalidade de $X$, podemos tomar $U$ vizinhança aberta de $x$ tal que $\bar{U} \subseteq V$. Tome $a=\{n \in$ $\left.\omega: x_{n} \in U\right\}$ e sejam $W_{1}=\overline{\left\{x_{n}: n \in a\right\}}$ e $W_{2}=\overline{\left\{x_{n}: n \in \omega \backslash a\right\}}$. Temos que $W_{1} \cup W_{2}=X$ e, por $\left.i i\right), W_{1} \cap W_{2}=\emptyset$. Logo $W_{1}$ é um aberto-fechado. Observe que $W_{2} \subseteq X \backslash U$, logo $U \subseteq W_{1}$. Portanto $x \in W_{1} \subseteq \bar{U} \subseteq V$, concluindo a afirmação.

Afirmação 2.4.2. Se u é um ultrafiltro sobre $\omega$, então

$$
\bigcap_{a \in u} \overline{\left\{x_{n}: n \in a\right\}}
$$

é unitário.

Notemos que $\overline{\left\{x_{n}: n \in a \cap b\right\}} \subseteq \overline{\left\{x_{n}: n \in a\right\}} \cap \overline{\left\{x_{n}: n \in b\right\}}$ para todos $a, b \subseteq \omega$. Assim, se $u$ é um ultrafiltro sobre $\mathbb{N}$, a família $\left\{\overline{\left\{x_{n}: n \in a\right\}}: a \in u\right\}$ tem a propriedade de intersecção finita. Pela compacidade de $X$ temos que $\bigcap_{a \in u} \overline{\left\{x_{n}: n \in a\right\}} \neq \emptyset$. Mostraremos que é unitário. 
Suponha que $\bigcap_{a \in u} \overline{\left\{x_{n}: n \in a\right\}}$ tenha dois elementos distintos, a saber, $x$ e $y$. Pela afirmação 2.4.1, e como $X$ é Hausdorff, existe $a \subseteq \omega$ tal que $x \in \overline{\left\{x_{n}: n \in a\right\}}$ e $y \in \overline{\left\{x_{n}: n \in \omega \backslash a\right\}}$. Como $u$ é um ultrafiltro, ou $a \in u$, ou $\omega \backslash a \in u$, contradizendo que ambos $x$ e $y$ pertencem a $\bigcap_{a \in u} \overline{\left\{x_{n}: n \in a\right\}}$, provando a afirmação.

Defina $\phi: \beta \mathbb{N} \longrightarrow X$ por $\phi(u)$ é o único elemento de $\bigcap_{a \in u} \overline{\left\{x_{n}: n \in a\right\}}$. Precisamos mostrar que $\phi$ é injetora e é contínua.

Se $u$ e $v$ são elementos distintos de $\beta \mathbb{N}$, existe $a \subseteq \omega$ tal que $a \in u$ e $\omega \backslash a \in v$. Temos $\phi(u) \in \overline{\left\{x_{n}: n \in a\right\}}$ e $\phi(v) \in \overline{\left\{x_{n}: n \in \omega \backslash a\right\}}$. Por $\left.i i\right)$ temos $\phi(u) \neq \phi(v)$, provando que $\phi$ é injetora.

Para mostrar a continuidade de $\phi$, pela afirmação 2.4.1 basta mostrar que $\phi^{-1}\left[\overline{\left\{x_{n}: n \in a\right\}}\right]$ é aberto, para cada $a \subseteq \omega$. Mas $\phi^{-1}\left[\overline{\left\{x_{n}: n \in a\right\}}\right]=\{u \in$ $\beta \mathbb{N}: a \in u\}=a^{*}$, que é aberto em $\beta \mathbb{N}$.

A aplicação $\phi$ é aberta sobre a imagem, pois é contínua e injetora, e $\beta \mathbb{N}$ é compacto. Como $\phi\left(n^{*}\right)=x_{n}$ temos que $\phi[\beta \mathbb{N}]=X$. Portanto $\phi$ é um homeomorfismo sobre $X$.

Teorema $2.5(\mathrm{CH})$. Existe um espaço compacto conexo $K$ tal que $C(K)$ é indecomponível e $K$ contém $\beta \mathbb{N}$.

Demonstração: Como em [Ko2], construímos por indução espaços compactos conexos $\left(K_{\alpha}\right)_{\alpha \leq \omega_{1}}$, seqüências de pontos $\left\{q_{n} \mid \alpha: n \in \omega\right\} \subseteq K_{\alpha}$, e conjuntos $b_{\alpha} \subseteq a_{\alpha} \subseteq \omega$, para $\alpha<\omega_{1}$. Construímos $\left(K_{\alpha}\right)_{\alpha \leq \omega_{1}}$ de modo que $K_{0}=[0,1]^{2}, K_{\alpha+1}$ é uma extensão forte de $K_{\alpha}$ por uma seqüência de funções contínuas duas a duas disjuntas e $K_{\alpha}$ é o limite inverso de $\left(K_{\beta}\right)_{\beta<\alpha}$, no caso $\alpha$ limite. Temos, ainda, em cada passo $\alpha, b_{\beta} \subseteq a_{\beta} \subseteq \omega$, para $\beta<\alpha$, e $\left\{q_{n} \mid \alpha: n \in \omega\right\}$ denso em $K_{\alpha}$ tais que $\left\{q_{n} \mid \alpha: n \in a_{\beta}\right\}$ é relativamente discreto e

$$
\left\{q_{n} \mid \alpha: n \in b_{\beta}\right\} \cap\left\{q_{n} \mid \alpha: n \in a_{\beta} \backslash b_{\beta}\right\} \neq \emptyset,
$$

em $K_{\alpha}$.

Em $K_{0}=[0,1]^{2}$ seja $\left\{x_{n} \mid 0: n \in \omega\right\}$ uma seqüência relativamente discreta e disjunta de $\left\{q_{n} \mid 0: n \in \omega\right\}$. Sejam Par o conjunto dos ordinais pares de $\omega_{1} \mathrm{e}$ Impar o conjunto dos ordinais ímpares de $\omega_{1}$. Em cada $K_{\alpha}$ obtemos $\left\{x_{n} \mid \alpha\right.$ : $n \in \omega\}$ relativamente discreto que estendem $x_{n} \mid 0$. Na construção indutiva, se $\alpha \in$ Par procedemos como em [Ko2] para obter $K_{\alpha+1}$, identificando Par com $\omega_{1}$. Fixemos uma enumeração $\left\{N_{\alpha}: \alpha \in\right.$ Impar $\}$ de todos os subconjuntos de 
$\omega$. Seja $\alpha \in$ Impar. Por $\mathrm{CH}$ temos que $K_{\alpha}$ tem peso enumerável, e não tem pontos isolados porque é conexo. Portanto, podemos aplicar o Lema 1.22 para $K_{\alpha}$, obtendo funções duas a duas disjuntas $f_{n}: K_{\alpha} \longrightarrow[0,1]$, para $n \in N_{\alpha}$, tais que $f_{n}\left(x_{m} \mid \alpha\right)=1$, se $n=m, f_{n}\left(x_{m} \mid \alpha\right)=0$, se $n \neq m$, e a extensão de $K_{\alpha}$ por $\left(f_{n}\right)_{n \in N_{\alpha}}$ é forte. Defina $K_{\alpha+1}$ como essa extensão, e $x_{n} \mid(\alpha+1)=\left(x_{n} \mid \alpha, 1\right)$, se $n \in N_{\alpha}$, e $x_{n} \mid(\alpha+1)=\left(x_{n} \mid \alpha, 0\right)$, se $n \notin N_{\alpha}$. Temos que

$$
\overline{\left\{x_{n} \mid(\alpha+1): n \in N_{\alpha}\right\}} \cap \overline{\left\{x_{n} \mid(\alpha+1): n \in \omega \backslash N_{\alpha}\right\}}=\emptyset,
$$

em $K_{\alpha+1}$. No caso $\alpha \in P$ ar, tome $x_{n} \mid(\alpha+1)$ qualquer extensão de $x_{n}(\alpha)$.

Observe que, para todos $\beta<\alpha<\omega_{1}$, temos

$$
\overline{\left\{x_{n} \mid \alpha: n \in N_{\beta}\right\}} \cap \overline{\left\{x_{n} \mid \alpha: n \in \omega \backslash N_{\beta}\right\}}=\emptyset,
$$

em $K_{\alpha}$. Tomando $x_{n}=\cup_{\alpha<\omega_{1}} x_{n} \mid \alpha$ temos que, para todo $a \subseteq \omega, \overline{\left\{x_{n}: n \in a\right\}} \cap$ $\overline{\left\{x_{n}: n \in \omega \backslash a\right\}}=\emptyset$, em $K=K_{\omega_{1}}$. Pelo Lema 2.4 temos que $K$ contém um subespaço homeomorfo a $\beta \mathbb{N}$. 


\section{Capítulo 3}

\section{Outras construções de $C(K)$ com poucos operadores}

Neste capítulo, mostraremos outras construções de espaços de Banach $C(K)$ com poucos operadores.

Na Seção 3.1 mostramos que existe um espaço $C(K)$ em que todo operador é multiplicador fraco mas nem todo é da forma $g I+S$, onde $g \in C(K)$ e $S$ é fracamente compacto. O mesmo resultado foi obtido independentemente em [Schl], com a mesma construção mas outra demonstração.

$\mathrm{Na}$ Seção 3.2, construímos $2^{\omega}$ espaços de Banach indecomponíveis da forma $C(K)$ essencialmente incomparáveis.

Retomamos a definição de que um espaço topológico é 0-dimensional se possui uma base de abertos-fechados. Para espaços Hausdorff compactos, isso é equivalente a totalmente desconexo, isto é, não contém nenhum subespaço conexo com mais de um elemento.

\subsection{Espaços de Banach $C(K)$ com quase pou- cos operadores}

Do Lema 1.5 segue que se um operador em um espaço $C(K)$ é da forma $g I+S$, para $g \in C(K)$, $I$ é a identidade e $S$ é fracamente compacto, então ele é multiplicador fraco. Em [Ko2] mostra-se que um operador $T: C(K) \longrightarrow C(K)$ é multiplicador fraco se, e somente se, o operador adjunto $T^{*}: M(K) \longrightarrow$ $M(K)$ é da forma $g I+S$, onde $S$ é fracamente compacto, $g$ é uma função 
boreliana de $K$ em $\mathbb{R}$ e, para $\mu \in M(K), g I(\mu) \in M(K)$ é definida por $g I(\mu)(E)=\int_{E} g d \mu$.

Nesta Seção apresentaremos uma construção de um espaço compacto $K$ tal que todo operador em $C(K)$ é um multiplicador fraco, mas existe operador em $C(K)$ que não é da forma $g I+S$, para $g \in C(K)$ e $S$ fracamente compacto.

Seja $K$ um compacto 0-dimensional tal que todo operador em $C(K)$ é da forma $g I+S$, onde $g \in C(K), I$ é o operador identidade e $S$ é um operador fracamente compacto.

Sejam $K_{1}, K_{2}$ abertos-fechados disjuntos de $K$. Temos o seguinte lema:

Lema 3.1. Se $T: C\left(K_{1}\right) \longrightarrow C\left(K_{2}\right)$ é um operador linear, então $T$ é fracamente compacto.

Demonstração: Defina $T_{1}: C(K) \longrightarrow C(K)$ tal que, para cada $f \in$ $C(K), T_{1}(f): K \longrightarrow \mathbb{R}$ é dada por $T_{1}(f)(x)=T\left(\left.f\right|_{K_{1}}\right)(x)$, se $x \in K_{2}$, e $T_{1}(f)(x)=0$, se $x \in K \backslash K_{2}$.

Como $C(K)$ tem poucos operadores, $T_{1}=g I+S$, para algum $g \in C(K)$ e $S$ fracamente compacto.

Seja $\left(f_{n}\right)_{n \in \omega}$ uma seqüência limitada em $C\left(K_{1}\right)$ tal que $f_{n} \cdot f_{n}=0$, para $n \neq m$. Pelo Lema 1.5, para concluir que $T$ é fracamente compacto basta mostrarmos que $\left\|T\left(f_{n}\right)\right\|$ converge a 0 .

Para cada $n \in \omega$ definimos $\tilde{f}_{n}: K \longrightarrow \mathbb{R}$ estendendo $f_{n}$ por 0 nos pontos fora de $K_{1}$. Como $K_{1}$ é aberto-fechado em $K, \tilde{f}_{n} \in C(K)$.

Pela definição de $T_{1}$, temos que $T\left(f_{n}\right)=\left.T_{1}\left(\tilde{f}_{n}\right)\right|_{K_{2}}$. Por outro lado $T_{1}\left(\tilde{f}_{n}\right)=g \tilde{f}_{n}+S\left(\tilde{f}_{n}\right)$. Como $\left.f_{n}\right|_{K_{1}}=0$, temos $\left.T\left(f_{n}\right) \stackrel{\left.\tilde{f}_{n}\right)}{=} S\left(\tilde{f}_{n}\right)\right|_{K_{2}}$. Como $S$ é fracamente compacto, pelo Lema 1.5 temos que $\left\|S\left(\tilde{f}_{n}\right)\right\|$ converge a 0 e, portanto, $\left\|T\left(f_{n}\right)\right\|$ converge a 0 .

Teorema 3.2. Existe um espaço compacto 0-dimensional $L$ tal que todo operador em $C(L)$ é multiplicador fraco mas nem todo operador é da forma $g I+S$, para algum $g \in C(L)$ e $S$ é fracamente compacto.

Demonstração: Fixemos pontos $x_{1} \in K_{1}$ e $x_{2} \in K_{2}$. Defina uma relação de equivalência $\sim$ sobre $K_{1} \cup K_{2}$ dada por $x \sim y$ se, e somente se, $x=y$ ou $\{x, y\}=\left\{x_{1}, x_{2}\right\}$.

Tomemos $L=K_{1} \cup K_{2} / \sim$ e defina em $L$ a seguinte topologia: $V \subseteq L$ é aberto se, e somente se, $\{x:[x] \in V\} \cap K_{1}$ é aberto em $K_{1}$ e $\{x:[x] \in V\} \cap K_{2}$ é aberto em $K_{2}$. 
Notemos que $L$ está bem-definido como espaço topológico, é compacto, e $K_{1}$ e $K_{2}$ estão contidos homeomorficamente em $K$ pela relação $x \mapsto[x]$.

Pela compacidade de $K_{1}$ e $K_{2}$, sabemos que eles são, com a identificação acima, subespaços fechados de $L$. Tomando $U=K_{1} \backslash\left\{x_{1}\right\}$ e $V=K_{2} \backslash\left\{x_{2}\right\}$, temos que $U$ e $V$ são abertos disjuntos, e $\bar{U} \cap \bar{V}=\left\{\left[x_{1}\right]\right\}=\left\{\left[x_{2}\right]\right\}$. Como $U$ e $V$ são abertos-fechados em $L \backslash\left\{\left[x_{1}\right]\right\}$, definindo $f: L \backslash\left\{\left[x_{1}\right]\right\} \longrightarrow[0,1]$ por $\left.f\right|_{U}=0$ e $\left.f\right|_{V}=1$, temos que $f$ é contínua e não se estende continuamente a $L$. Logo $L \backslash\left\{\left[x_{1}\right]\right\}$ não é $C^{*}$-imerso em $L$. Pelo Teorema 1.11 , existe um operador em $C(L)$ que não é da forma $g I+S$. Mostraremos que todo operador em $C(L)$ é multiplicador fraco.

Seja $T: C(L) \longrightarrow C(L)$ um operador linear, e suponha que não seja multiplicador fraco. Seja $\left(e_{n}\right)_{n \in \omega}$ uma seqüência em $C(L)$ limitada e duas a duas disjuntas e sejam $x_{n} \in L$ e $\varepsilon>0$ tais que $e_{n}\left(x_{n}\right)=0$ e $\left|T\left(e_{n}\right)\left(x_{n}\right)\right|>$ $\varepsilon$, para todo $n$. Passando para uma subseqüência, podemos assumir que $e_{n}\left(\left[x_{1}\right]\right)=0$, para todo $n$.

Para cada $f \in C\left(K_{1}\right)$ definimos $\bar{f} \in C(L)$ por $\bar{f}([x])=f(x)$, se $x \in K_{1}$, e $\bar{f}([x])=f\left(x_{1}\right)$, se $x \in K_{2}$. Analogamente definimos $\bar{f} \in C(L)$ para $f \in$ $C\left(K_{2}\right)$. Notemos que, como $e_{n}\left(\left[x_{1}\right]\right)=0$, temos $e_{n}=\overline{e_{n \mid K_{1}}}+\overline{e_{n \mid K_{2}}}$, para todo $n$.

Para cada par $i, j \in\{1,2\}$ definimos uma transformação linear $T_{i, j}$ : $C\left(K_{i}\right) \longrightarrow C\left(K_{j}\right)$ dada por $T_{i, j}(f)=\left.T(\tilde{f})\right|_{K_{j}}$.

Passando a uma subseqüência podemos assumir que $x_{n} \in K_{1}$, para todo $n$. Temos

$T\left(e_{n}\right)\left(x_{n}\right)=T\left(\overline{e_{n \mid K_{1}}}\right)\left(x_{n}\right)+T\left(\overline{e_{n \mid K_{2}}}\right)\left(x_{n}\right)=T_{11}\left(\left.e_{n}\right|_{K_{1}}\right)\left(x_{n}\right)+T_{21}\left(\left.e_{n}\right|_{K_{2}}\right)\left(x_{n}\right)$.

Pelo Lema 3.1 o operador $T_{21}$ é fracamente compacto. Logo, do Lema 1.5 temos que $T_{21}\left(\left.e_{n}\right|_{K_{2}}\right)\left(x_{n}\right)$ converge a 0 . Portanto concluímos que $\left|T_{11}\left(\left.e_{n}\right|_{K_{1}}\right)\left(x_{n}\right)\right|>$ $\varepsilon$, para infinitos $n$ 's, contradizendo que todo operador em $C\left(K_{1}\right)$ é multiplicador fraco.

\subsection{Construção de $2^{\omega}$ espaços $C(K)$ indecom- poníveis essencialmente incomparáveis}

Iremos construir uma família de $2^{\omega}$ espaços de Banach indecomponíveis da forma $C(K)$ dois a dois essencialmente incomparáveis.

Lembramos que um operador $T$ em um espaço de Banach $X$ é de Fredholm se a dimensão do núcleo e a codimensão da imagem são finitos (vide [DS]). 
Definição 3.3. Dois espaços de Banach $X$ e $Y$ são essencialmente incomparáveis se, para todos operadores $T: X \longrightarrow Y$ e $S: Y \longrightarrow X$, o operador $I-S \circ T: X \longrightarrow X$ é um operador de Fredholm.

Lema 3.4. Sejam $K$ e $L$ compactos tais que todo operador $T: C(K) \longrightarrow$ $C(L)$ é fracamente compacto. Então $C(K)$ e $C(L)$ são essencialmente incomparáveis.

Demonstração: Sabemos que a composição de um operador fracamente compacto com qualquer operador contínuo é um operador fracamente compacto. Por um resultado de [LT] temos que a soma de um operador de Fredholm e um operador estritamente singular é um operador de Fredholm. Pelo resultado de [Pe2], um operador fracamente compacto em $C(K)$ é estritamente singular. O operador identidade é de Fredholm, já que $\operatorname{dim}(\operatorname{Ker}(I))=$ $\operatorname{codim}(\operatorname{Im}(I))=0$. Logo, para $K$ e $L$ como na hipótese do lema, $I-S \circ T$ é um operador de Fredholm em $C(K)$, para todos $T: C(K) \longrightarrow C(L)$ e $S: C(L) \longrightarrow C(K)$.

Observamos que para o Lema 3.4 assumimos que todo operador é fracamente compacto apenas em uma direção, de $C(K)$ em $C(L)$, e concluímos que $C(K)$ e $C(L)$ são essencialmente incomparáveis. A existência de um operador não fracamente compacto de $C(K)$ em $C(L)$ não implica a existência de um operador não fracamente compacto de $C(L)$ em $C(K)$.

Teorema 3.5. Existem espaços compactos conexos $\left(K_{\xi}\right)_{\xi<2^{\omega}}$ tais que, para todo $\xi<2^{\omega}, C\left(K_{\xi}\right)$ é indecomponível e, para todos $\xi, \eta<2^{\omega}$ tais que $\xi \neq \eta$, todo operador $T: C\left(K_{\xi}\right) \longrightarrow C\left(K_{\eta}\right)$ é fracamente compacto.

Demonstração: Fixe uma enumeração $\left(\xi(\alpha), \eta(\alpha), f_{n}(\alpha), g_{n}(\alpha), l_{n}(\alpha)\right)_{\alpha<2^{\omega}, n \in \omega}$ tal que, para todo $\beta<2^{\omega}$, dados

- $\xi, \eta<2^{\omega}$

- $f_{n}:[0,1]^{2^{\omega}} \longrightarrow[0,1]$ contínuas e duas a duas disjuntas, para $n \in \omega$;

- $g_{n}:[0,1]^{2^{\omega}} \longrightarrow \mathbb{R}$ contínuas;

- $l_{n}$ uma seqüência crescente de inteiros positivos; 
existe $\alpha>\beta$ tal que $\xi(\alpha)=\xi, \eta(\alpha)=\eta, f_{n}(\alpha)=f_{n}, g_{n}(\alpha)=g_{n}$ e $l_{n}(\alpha)=l_{n}$, para todo $n \in \omega$.

Construiremos, por indução em $\alpha<2^{\omega}$, espaços compactos $K_{\xi}(\alpha)$, seqüências de pontos $\left(q_{\xi}^{n} \mid \alpha\right)_{n \in \omega} \subseteq K_{\xi}(\alpha)$, e conjuntos $b_{\xi}(\alpha) \subseteq a_{\xi}(\alpha) \subseteq \omega$, para $\xi<2^{\omega}$. A construção será feita de modo que, para todos $\beta \leq \alpha<2^{\omega}$ e todo $\xi<2^{\omega}$, $\left\{q_{\xi}^{n} \mid \alpha: n \in a_{\xi}(\beta)\right\}$ é relativamente discreto e

$$
\overline{\left\{q_{\xi}^{n} \mid \alpha: n \in b_{\xi}(\beta)\right\}} \cap \overline{\left\{q_{\xi}^{n} \mid \alpha: n \in a_{\xi}(\beta) \backslash b_{\xi}(\beta)\right\}} \neq \emptyset,
$$

em $K_{\xi}(\alpha)$.

O plano desta demonstração é o seguinte: para os casos em que $\xi(\alpha)=$ $\eta(\alpha)$, iremos proceder como em [Ko2], eliminando os operadores em $C\left(K_{\xi(\alpha)}\right)$ que não são multiplicadores fracos. Para $\xi(\alpha) \neq \eta(\alpha)$, por um argumento similar eliminaremos os operadores de $C\left(K_{\xi(\alpha)}\right)$ em $C\left(K_{\eta(\alpha)}\right)$ que não são fracamente compactos.

Para cada $\xi<2^{\omega}$, definimos $K_{\xi}(0)=[0,1]^{2}$ e $\left\{q_{\xi}^{n} \mid 0: n \in \omega\right\}$ uma enumeração de um conjunto enumerável denso em $[0,1]^{2}$. Se $\alpha>0$ é um ordinal limite, definimos $K_{\xi}(\alpha)$ o limite inverso de $\left(K_{\xi}(\beta)\right)_{\beta<\alpha}$, e $q_{\xi}^{n} \mid \alpha=$ $\cup_{\beta<\alpha} q_{\xi}^{n} \mid \beta$. Isso está bem definido pois construiremos cada $q_{\xi}^{n} \mid \beta$ como uma extensão de $q_{\xi}^{n} \mid \beta^{\prime}$, para $\beta^{\prime}<\beta$. Observe que, para $\alpha \geq \omega, K_{\xi}(\alpha) \subseteq[0,1]^{\alpha}$ e, para $\alpha<\omega, K_{\xi}(\alpha) \subseteq[0,1]^{\alpha+2}$. Como no Capítulo 2, por abuso de notação consideraremos sempre $K_{\xi}(\alpha) \subseteq[0,1]^{\alpha}$.

Suponha definidos $K_{\xi}(\alpha),\left(a_{\xi}(\beta)\right)_{\beta<\alpha},\left(b_{\xi}(\beta)\right)_{\beta<\alpha}$ e $q_{\xi}^{n} \mid \alpha$, para todos $\xi<$ $2^{\omega}$ e $n \in \omega$. Se $\xi \neq \xi(\alpha)$ definimos $K_{\xi}(\alpha+1)=K_{\xi}(\alpha) \times\{0\}$ e $q_{\xi}^{n} \mid(\alpha+$ $1)=q_{\xi}^{n} \mid \alpha \frown 0$. Para $\eta \neq \eta(\alpha)$ definimos $a_{\eta}(\alpha)=a_{\eta}(0)$ e $b_{\eta}(\alpha)=b_{\eta}(0)$, no caso $\alpha>0$. Se $\alpha=0$ tomamos $a_{\eta}(\alpha)$ e $b_{\eta}(\alpha)$ quaisquer de modo que $\left\{q_{\eta}^{n}(\alpha): n \in a_{\eta}(\alpha)\right\}$ seja relativamente discreto em $K_{\xi}(\alpha)$ e $(*)$ valha para $\alpha=\beta$ (é possível, pois $[0,1]^{2}$ é métrico).

Iremos definir $K_{\xi(\alpha)}(\alpha+1),\left\{q_{\xi(\alpha)}^{n}(\alpha+1): n \in \omega\right\}, b_{\eta(\alpha)}(\alpha)$ e $a_{\eta(\alpha)}(\alpha)$. Diremos que um passo $\alpha$ é não-trivial se

1. existem $f_{n}^{\prime}: K_{\xi(\alpha)}(\alpha) \longrightarrow[0,1]$, para $n \in \omega$, tais que, se $y \mid \alpha=x \in$ $K_{\xi(\alpha)}(\alpha)$, para $y \in[0,1]^{2^{\omega}}$, então $f_{n}(\alpha)(y)=f_{n}^{\prime}(x)$;

2. $\left\{q_{\eta(\alpha)}^{l_{n}} \mid \alpha: n \in \omega\right\}$ é relativamente discreto em $K_{\eta(\alpha)}(\alpha)$;

3. $f_{n}^{\prime}\left(q_{\xi(\alpha)}^{l_{m}} \mid \alpha\right)=0$, para todos $n, m \in \omega$, se $\xi(\alpha)=\eta(\alpha)$;

4. se $\xi(\alpha) \neq \eta(\alpha)$, existem $g_{n}^{\prime}: K_{\eta(\alpha)}(\alpha) \longrightarrow \mathbb{R}$, para $n \in \omega$, tais que, se $y \mid \alpha=x \in K_{\eta(\alpha)}(\alpha)$, para $y \in[0,1]^{2^{\omega}}$, então $g_{n}(\alpha)(y)=g_{n}^{\prime}(x)$; 
5. existe $\varepsilon>0$ tal que $\left|g_{n}^{\prime}\left(q_{\eta(\alpha)}^{l_{m}} \mid \alpha\right)\right|>\varepsilon$, se $\xi(\alpha) \neq \eta(\alpha)$.

Se $\alpha$ é um passo trivial, procedemos nas coordenadas $\xi(\alpha)$ e $\eta(\alpha)$ como nos casos $\xi \neq \xi(\alpha), \eta(\alpha)$, para definirmos $K_{\xi}(\alpha+1), q_{\xi}^{n} \mid(\alpha+1), a_{\eta}(\alpha)$ e $b_{\eta}(\alpha)$.

Seja $\alpha$ um passo não-trivial. Pelo Lema 1.19 tomamos $a \subseteq \omega$ tal que, para todo $b \subseteq a$, a extensão de $K_{\xi(\alpha)}(\alpha)$ por $\left(f_{n}^{\prime}\right)_{n \in b}$ é forte e $(*)$ é preservado em $\alpha+1$, para todo $\beta<\alpha$ e $\xi=\xi(\alpha)$, se tomarmos

$$
q_{\xi(\alpha)}^{n}\left|(\alpha+1)=q_{\xi(\alpha)}^{n}\right| \alpha \frown \Sigma_{m \in b} f_{m}^{\prime}\left(q_{\xi(\alpha)}^{n} \mid \alpha\right) .
$$

Notemos que, no caso $\xi(\alpha) \neq \eta(\alpha)$, já definimos $a_{\xi(\alpha)}(\alpha)$ e $b_{\xi(\alpha)}(\alpha)$, e devemos escolher $a$ preservando $(*)$ também para $\beta=\alpha$.

Tome $a_{\eta(\alpha)}(\alpha)=\left\{l_{n}: n \in a\right\}$. Como $K_{\xi(\alpha)}(\alpha)$ tem peso $\alpha<2^{\omega}$ e dois fechados disjuntos em um espaço compacto podem ser separados por uma quantidade finita de abertos básicos, podemos escolher $b \subseteq a_{\eta(\alpha)}(\alpha)$ tal que

$$
\overline{\left\{q_{\eta(\alpha)}^{n} \mid \alpha: n \in b\right\}} \cap \overline{\left\{q_{\eta(\alpha)}^{n} \mid \alpha: n \in a_{\eta(\alpha)}(\alpha) \backslash b\right\}} \neq \emptyset .
$$

Defina $b_{\eta(\alpha)}(\alpha)=b, K_{\xi(\alpha)}(\alpha+1)$ a extensão forte de $K_{\xi(\alpha)}(\alpha)$ por $\left(f_{n}^{\prime}\right)_{n \in b_{\eta(\alpha)}(\alpha)}$ e $q_{\xi(\alpha)}^{n} \mid(\alpha+1)$ como em $(* *)$.

No caso $\xi(\alpha)=\eta(\alpha)$ temos que verificar a preservação de $(*)$ para $a_{\xi(\alpha)}(\alpha)$ e $b_{\xi(\alpha)}(\alpha)$. Como $f_{n}^{\prime}\left(q_{\xi}^{l_{m}}\right)=0$, para todos $n, m \in \omega$, se $x$ pertence à intersecção em $(\dagger)$, temos

$$
(x, 0) \in \overline{\left\{q_{\xi(\alpha)}^{n} \mid \alpha \frown 0: n \in b\right\}} \cap \overline{\left\{q_{\eta(\alpha)}^{n} \mid \alpha \frown 0: n \in a_{\eta(\alpha)}(\alpha) \backslash b\right\}},
$$

de onde concluímos que (*) é preservado no passo $\alpha+1$, para todo $\beta \leq \alpha$.

Tomamos $K_{\xi}$ o limite inverso de $\left(K_{\xi}(\alpha)\right)_{\alpha<2^{\omega}}$, para cada $\xi<2^{\omega}$. Pelo item a) do Lema 1.17, se $\left\{q_{\xi}^{n} \mid \alpha: n \in \omega\right\}$ é denso em $K_{\xi}(\alpha)$ então $\left\{q_{\xi}^{n} \mid(\alpha+1)\right.$ : $n \in \omega\}$ é denso em $K_{\xi}(\alpha+1)$. Como densidade é preservada em limites inversos, por indução concluímos que $\left\{q_{\xi}^{n}: n \in \omega\right\}$ é denso em $K_{\xi}$, onde $q_{\xi}^{n}=\cup_{\alpha<2^{\omega}} q_{\xi}^{n} \mid \alpha$.

Pelo Lema 1.20 temos que $K_{\xi} \backslash F$ é conexo, para todo $F$ finito. Logo, pelo Teorema 1.9, basta mostrarmos que todo operador em $C\left(K_{\xi}\right)$ é multiplicador fraco, para concluirmos que $C\left(K_{\xi}\right)$ é indecomponível.

Afirmação 3.5.1. Para todos $\xi, \eta<2^{\omega}$, com $\xi \neq \eta$, todo operador $T$ : $C\left(K_{\xi}\right) \longrightarrow C\left(K_{\eta}\right)$ é fracamente compacto. 
Suponha que exista $T: C\left(K_{\xi}\right) \longrightarrow C\left(K_{\eta}\right)$ não fracamente compacto, para $\xi \neq \eta$. Pelo Lema 1.5, existe uma seqüência limitada $f_{n} \in C(K)$ de funções duas a duas disjuntas e $\varepsilon>0$ tais que $\left\|T\left(f_{n}\right)\right\|>\varepsilon$, para todo $n$. Considerando múltiplos de $\max \left(f_{n}, 0\right)$ e $-\min \left(f_{n}, 0\right)$, como na demonstração do Teorema 2.2, podemos assumir que $f_{n}$ tem imagem contida em [0,1].

Para cada $n$ podemos tomar $x_{n} \in K_{\eta}$ tal que $\left|T\left(f_{n}\right)\left(x_{n}\right)\right|>\varepsilon$. Como $\left\{q_{\eta}^{n}: n \in \omega\right\}$ é denso em $K_{\eta}$ podemos assumir que $x_{n}=q_{\xi}^{l_{n}}$, para inteiros $l_{n}$ 's. Observe que não podemos ter $l_{n}$ constante, para infinitos $n$ 's, pois, nesse caso, teríamos que somas finitas de $f_{n}$ 's formariam uma família limitada em $C\left(K_{\xi}\right)$ cujas imagens por $T$ teriam normas arbitrariamente grande, contradizendo a continuidade de $T$. Portanto, passando a uma subseqüência, podemos assumir que $\left(l_{n}: n \in \omega\right)$ é uma seqüência estritamente crescente e que $\left\{q_{\xi}^{l_{n}}: n \in \omega\right\}$ é relativamente discreto.

A partir de agora a demonstração é análoga à do Teorema 2.2. Iremos suprimir alguns detalhes que constam naquela demonstração.

Considere $\mu_{n}=T^{*}\left(\delta_{x_{n}}\right)$. Temos que

$$
\left|T\left(f_{n}\right)\left(x_{n}\right)\right|=\left|\int f_{n} d \mu_{n}\right|>\varepsilon .
$$

Aplicando o Lema de Rosenthal, achamos $N^{\prime} \subseteq \omega$ tal que

$$
\Sigma\left\{\left|\int f_{m} d \mu_{n}\right|: n \neq m, m \in N^{\prime}\right\}<\frac{\varepsilon}{3}
$$

Como está demonstrado em 2.2, existe $N^{\prime \prime} \subseteq N^{\prime}$ tal que, para todo $b \subseteq \omega$ e todo $n \in \omega$,

$$
\int \sup \left\{f_{m}: m \in b\right\} d \mu_{n}=\int \Sigma_{m \in b} f_{m} d \mu_{n}
$$

sempre que o supremo existe em $C(K)$.

Passando novamente a uma subseqüência, assumimos que $N^{\prime \prime}=\omega$.

Seja $g_{n}=T\left(f_{n}\right)$, para $n \in \omega$. Pelo Teorema de Mibu, aplicado a extensões de $f_{n}$ e $g_{n}$ para todo $[0,1]^{2 \omega}$, existem $\alpha^{\prime}<2^{\omega}$ e funções $f_{n}^{\prime}: K_{\alpha^{\prime}} \longrightarrow[0,1]$ e $g_{n}^{\prime}: K_{\alpha^{\prime}} \longrightarrow \mathbb{R}$ tais que $f_{n}=f_{n}^{\prime} \circ \pi$ e $g_{n}=g_{n}^{\prime} \circ \pi$. A existência de tais funções continua valendo para $\beta>\alpha^{\prime}$, pois basta tomarmos $f_{n}^{\prime} \circ \pi_{K_{\beta}, K_{\alpha^{\prime}}}$ no lugar de $f_{n}^{\prime}$.

Sejam $\tilde{f}_{n}:[0,1]^{2^{\omega}} \longrightarrow[0,1]$ e $\tilde{g}_{n}:[0,1]^{2^{\omega}} \longrightarrow[0,1]$ extensões de $f_{n_{\tilde{f}}}$ e $g_{n}$, respectivamente. Tome $\alpha>\alpha^{\prime}$ tal que $\xi(\alpha)=\xi, \eta(\alpha)=\eta, f_{n}(\alpha)=\tilde{f}_{n}$, $l_{n}(\alpha)=l_{n}$ e $g_{n}(\alpha)=\tilde{g}$. Temos que $\alpha$ é um passo não-trivial. 
Pela construção e o Lema 1.17, $\left\{f_{n}: n \in b_{\eta}(\alpha)\right\}$ tem supremo em $K_{\xi}$ e

$$
\overline{\left\{q_{\eta}^{l_{n}}: n \in b_{\eta}(\alpha)\right\}} \cap \overline{\left\{q_{\eta}^{l_{n}}: n \in a_{\beta}(\alpha) \backslash b_{\eta}(\alpha)\right\}} \neq \emptyset
$$

em $K_{\xi}$. Para facilitar a notação, denotaremos $a_{\beta}(\alpha)$ e $b_{\eta}(\alpha)$ por $a$ e $b$, respectivamente.

Tome $f=\sup \left\{f_{n}: n \in b\right\}$. Repetindo os cálculos feitos em 2.2, concluímos que

$$
\left|T(f)\left(q_{\eta}^{l_{n}}\right)\right| \geq \frac{2 \varepsilon}{3}
$$

se $n \in b$, e

$$
\left|T(f)\left(q_{\eta}^{l_{n}}\right)\right| \leq \frac{\varepsilon}{3},
$$

se $n \in a \backslash b$, contradizendo que $T(f)$ é contínua e $\overline{\left\{q_{\eta}^{l_{n}}: n \in b\right\}} \cap \overline{\left\{q_{\eta}^{l_{n}}: n \in a \backslash b\right\}} \neq$ $\emptyset$.

Afirmação 3.5.2. Para todo $\xi<2^{\omega}$, todo operador em $C\left(K_{\xi}\right)$ é multiplicador fraco.

Suponha que exista $T: C\left(K_{\xi}\right) \longrightarrow C\left(K_{\xi}\right)$ que não é multiplicador fraco. Então existem $f_{n} \in C\left(K_{\xi}\right)$ duas a duas disjuntas e $x_{n} \in K_{\xi}$ tais que $f_{n}\left(x_{n}\right)=$ 0 e $\left|T\left(f_{n}\right)\left(x_{n}\right)\right|>\varepsilon$, para algum $\varepsilon>0$. Pela densidade de $\left\{q_{\xi}^{n}: n \in \omega\right\}$ em $K_{\xi}$, podemos assumir que $x_{n}=q_{\xi}^{l_{n}}$. O resto da demonstração é totalmente análoga às da Afirmação 3.5.1 e do Teorema 2.2.

A construção aqui apresentada pode ser repetida em qualquer construção indutiva, de comprimento $2^{\omega}$, em que tomamos sucessivas extensões fortes. Adaptando as técnicas usadas neste capítulo nas construções feitas nas Seções 2.1 , 3.1 e 2.2 , obtemos a existência de $2^{\omega}$ espaços $C(K)$ indecomponíveis essencialmente incomparáveis tais que

1. $(\diamond) C(L)$ tem poucos operadores, para todo $L \subseteq K$;

2. $(\mathrm{CH}) K$ contém $\beta \mathbb{N}$;

3. (CH) todo operador em $C(K)$ é da forma $g I+S$, para algum $g \in C(K)$ e $S$ fracamente compacto; 


\section{Capítulo 4}

\section{Um espaço $C(K)$ de densidade $\omega_{1}<2^{\omega}$ com poucos operadores}

Os espaços $C(K)$ com poucos operadores construídos em [Ko2], [Pl] e nos Capítulos 2 e 3 têm densidade $2^{\omega}$. Neste capítulo, usando a técnica de forcing, mostramos a consistência da existência de um espaço de Banach $C(K)$ de densidade menor que contínuo com poucos operadores.

Em [Ko2] mostra-se que espaços de Banach da forma $C(K)$ com poucos operadores possuem a propriedade de Grothendieck. Espaços da forma $C(K)$ separáveis não são de Grothendieck, pois $C(K)$ separável implica que $K$ é metrizável e, portanto, contém seqüência convergente não-trivial, implicando que $c_{0}$ é complementado em $C(K)$. Logo $\mathrm{CH}$ implica que não existe espaço de Banach $C(K)$ de densidade menor que contínuo (no caso, enumerável) com poucos operadores.

Podemos perguntar se a existência de $C(K)$ de densidade menor que contínuo com poucos operadores não é equivalente a $\neg \mathrm{CH}$. Por outro lado, podemos perguntar se podemos provar, em ZFC, que existe $C(K)$ de densidade $\omega_{1}$ com poucos operadores. A resposta é não. Define-se $\mathfrak{p}$ o menor cardinal para o qual existe uma família $\left(M_{\alpha}\right)_{\alpha<\mathfrak{p}}$ de subconjuntos de $\omega$ tal que $\bigcap_{\alpha \in F} M_{\alpha}$ é infinito, para todo $F \subseteq \mathfrak{p}$ finito, e, para todo $M \subseteq \omega$, existe $\alpha<\kappa$ tal que $M \backslash M_{\alpha}$ é finito. Em [Fr] está provado que $\mathrm{MA}+\neg \mathrm{CH}$ implica $\mathfrak{p}=2^{\omega}$ e que todo compacto $K$ de peso menor que $\mathfrak{p}$ contém seqüência convergente não trivial, o que implica que $C(K)$ não possui a propriedade de Grothendieck. Portanto, a construção de $C(K)$ apresentada neste capítulo é independente de ZFC, mesmo assumindo a negação de $\mathrm{CH}$. Isto é, concluímos que usando apenas os axiomas de $\mathrm{ZFC} \mathrm{e} \neg \mathrm{CH}$ não podemos provar 
nem refutar que existe um espaço $C(K)$ de densidade $\omega_{1}<2^{\omega}$ com poucos operadores.

A primeira construção consistente de um espaço de Banach $C(K)$ de densidade menor que contínuo com a propriedade de Grothendieck foi obtida recentemente por Brech $([\mathrm{Br}])$. Vejamos que o espaço $C(K)$ construído em $[\mathrm{Br}]$ tem operadores que não são multiplicadores fracos.

O compacto $K$ construído em [Br] é o espaço de Stone, em $M[G]$, da álgebra de Boole $\mathcal{P}(\omega) \cap M$, onde $M$ é um modelo transitivo enumerável para ZFC e $M[G]$ é a extensão genérica de $M$ por um $P$-genérico $G$ sobre $M$, para um determinado forcing $P \in M$ (veja Apêndiec B). O modelo $M[G]$ satisfaz que $C(K)$ é de Grothendieck e tem densidade $\omega_{1}<2^{\omega}$.

Como $M$ é um modelo para ZFC, se $a \subseteq \omega$ pertence a $M$ então o conjunto $\{2 n: n \in a\}$ também pertence a $M$. Portanto a álgebra $A=\mathcal{P}(\omega) \cap M$, que é uma subálgebra de $\mathcal{P}(\omega)$, tem a propriedade de que $a \in A$ implica $\{2 n: n \in a\} \in A$. Isso gera um isomorfismo entre $A$ e a álgebra $\{a \cap(2 \omega)$ : $a \in A\}$, onde $2 \omega$ é o conjunto dos números pares. Desse isomorfismo segue que $K=S(A)$ e $(2 \omega)^{*}$ são homeomorfos e, portanto, $C(K)$ e $C\left((2 \omega)^{*}\right)$ são isomorfos. Como $(2 \omega)^{*}$ está contido propriamente em $K$, usando o Lema 1.8 e repetindo o argumento usado no Corolário 2.3, concluímos que $C(K)$ tem operadores que não são multiplicadores fracos.

\subsection{Convergência fraca em $M(K)$}

Apresentamos nesta seção lemas sobre a topologia fraca em $M(K)$, que serão usados neste capítulo e no Capítulo 5

Definição 4.1. Sejam $K$ um espaço topológico compacto e $\varepsilon>0$. Dizemos que um conjunto limitado $S \subseteq M(K)$ é $\varepsilon$-fracamente relativamente compacto se, para toda seqüência $\left(V_{n}\right)_{n \in \omega}$ de abertos de $K$ dois a dois disjuntos,

$$
\sup \left\{\left|\mu\left(V_{n}\right)\right|: \mu \in S\right\} \leq \varepsilon,
$$

para todos, exceto finitos, $n \in \omega$.

Lema 4.2. Sejam $K$ um espaço topológico compacto e $S \subseteq M(K)$ limitado. Temos

a) Sé fracamente relativamente compacto se, e somente se, $S$ é $\varepsilon$-fracamente relativamente compacto, para todo $\varepsilon>0$. 
b) Dado $\varepsilon>0, S$ não é $\varepsilon$-fracamente relativamente compacto se, e somente se, existem uma seqüência $\left(\mu_{n}\right)_{n \in \omega}$ em $S$ e uma seqüência $\left(V_{n}\right)_{n \in \omega}$ de abertos de $K$ dois a dois disjuntos tais que $\left|\mu_{n}\left(V_{n}\right)\right|>\varepsilon$, para todo $n \in \omega$.

Demonstração: Item a) é conseqüência imediata do Teorema de Dieudonné-Grothendieck. Para o item $b$ ), suponha que $S$ não é $\varepsilon$-fracamente relativamente compacto. Então existe uma seqüência $\left(V_{n}\right)_{n \in \omega}$ de abertos de $K$ dois a dois disjuntos tal que $\sup \left\{\left|\mu\left(V_{n}\right)\right|: \mu \in S\right\}>\varepsilon$, para infinitos $n$ 's. Passando a uma subseqüência, podemos assumir que isso ocorre para todo $n$. Portanto, para cada $n \in \omega$ encontramos $\mu_{n} \in S$ tal que $\left|\mu_{n}\left(V_{n}\right)\right|>\varepsilon$, como queríamos. Para a outra direção, se temos $\mu_{n} \in S$ e $V_{n}$ 's abertos disjuntos de $K$ tais que $\left|\mu_{n}\left(V_{n}\right)\right|>\varepsilon$, para todo $n \in \omega$, teremos $\sup \left\{\left|\mu\left(V_{n}\right)\right|: \mu \in S\right\}>\varepsilon$, para todo $n \in \omega$, e, portanto, $S$ não será $\varepsilon$-fracamente relativamente compacto.

Dizemos que uma seqüência $\left(\mu_{n}\right)_{n \in \omega}$ de medidas é duas a duas disjunta se existem borelianos $A_{n}$ 's dois a dois disjuntos tais que $\left|\mu_{n}\right|\left(K \backslash A_{n}\right)=0$, para todo $n$.

Lema 4.3. Seja $K$ um compacto e seja $D \subseteq K$ denso. Suponha que $T$ : $C(K) \longrightarrow C(K)$ não é um multiplicador fraco. Então existe uma seqüência $\left(x_{n}\right)_{n \in \omega}$ em $D$ tal que, para toda função boreliana limitada $f: K \longrightarrow \mathbb{R}$, $T^{*}\left(\delta_{x_{n}}\right)-f \delta_{x_{n}}$ não é fracamente relativamente compacto, em $M(K)$, onde $f \delta_{x}=f(x) \delta_{x} \in M(K)$.

Demonstração: Se $T$ não é multiplicador fraco, existe uma seqüência limitada $\left(e_{n}\right)_{n \in \omega}$ de funções duas a duas disjuntas de $K$ em $\mathbb{R}$, uma seqüência $\left(x_{n}\right)_{n \in \omega}$ de pontos distintos de $K$ e $\varepsilon>0$ tais que $e_{n}\left(x_{n}\right)=0$ e $\left|T\left(e_{n}\right)\left(x_{n}\right)\right|>$ $\varepsilon$, para todo $n$. Como $D$ é denso em $K$, podemos assumir que $x_{n} \in D$. Sejam $\mu_{n}=T^{*}\left(\delta_{x_{n}}\right)-f \delta_{x_{n}}$. Temos

$$
T\left(e_{n}\right)\left(x_{n}\right)=\int e_{n} d T^{*}\left(\delta_{x_{n}}\right)=\int e_{n} d \mu_{n}+\int e_{n} d\left(f \delta_{x_{n}}\right) .
$$

Como $e_{n}\left(x_{n}\right)=0$, temos que $\int e_{n} d\left(f \delta_{x_{n}}\right)=0$ e, portanto,

$$
\left|T\left(e_{n}\right)\left(x_{n}\right)\right|=\left|\int e_{n} d \mu_{n}\right|>\varepsilon,
$$


52 Um espaço $C(K)$ de densidade $\omega_{1}<2^{\omega}$ com poucos operadores

para todo $n$.

Tome $M$ um limitante para $\left(e_{n}\right)_{n \in \omega}$. Temos $\left|\mu_{n}\right|\left(\operatorname{supp}\left(e_{n}\right)\right)>\frac{\varepsilon}{M}$. Usando a definição de variação de medidas encontramos borelianos $U_{n}$ contidos em $\operatorname{supp}\left(e_{n}\right)$ tais que $\left|\mu_{n}\left(U_{n}\right)\right|>\frac{\varepsilon}{2 M}$. Pela regularidade de $\mu_{n}$ podemos assumir que $U_{n}$ são abertos dois a dois disjuntos. Como $T$ e $f$ são limitados temos que $\left(\mu_{n}\right)_{n \in \omega}$ é uma seqüência limitada em $M(K)$. Logo, pelo Teorema de Dieudonné-Grothendieck, implica que $\left(\mu_{n}\right)_{n \in \omega}$ não é fracamente relativamente compacto.

Usaremos dois resultados citados em [Ta] (lemas 1 e 2, respectivamente).

Proposição 4.4 (Pełczyński). Sejam $K$ um espaço compacto, $\varepsilon>0$ e $\left(\mu_{n}\right)_{n \in \omega}$ uma seqüência de medidas positivas de $K$, de norma 1 , e duas a duas disjuntas. Então existem uma subseqüência $\left(\mu_{n}^{\prime}\right)_{n \in \omega}$ de $\left(\mu_{n}\right)_{n \in \omega}$ e abertos dois a dois disjuntos $\left(U_{n}\right)_{n \in \omega}$ tais que $\mu_{n}^{\prime}\left(U_{n}\right) \geq 1-\varepsilon$, para todo $n \in \omega$.

Proposição 4.5 (Kadec, Pełczyński). Seja $(X, \Sigma, \mu)$ um espaço mensurável, onde $\mu$ é positiva e de norma 1 . Seja $\left(v_{n}\right)_{n \in \omega}$ uma seqüência limitada de $L^{1}(\mu)$. Então existe uma subseqüência $\left(v_{n}^{\prime}\right)_{n \in \omega}$ que se decompõe da forma $v_{n}^{\prime}=g_{n}+h_{n}$, onde $\left(g_{n}\right)_{n \in \omega}$ é fracamente convergente $e\left(h_{n}\right)_{n \in \omega}$ é duas a duas disjuntas.

A Demonstração da Proposição 4.4 encontra-se implícita na demonstração do Lema 1 de [Pe1]. A da Proposição 4.5 encontra-se no Teorema 6 de [KP].

Corolário 4.6. Seja $\left(\mu_{n}\right)_{n \in \omega}$ uma seqüência limitada de medidas em um compacto $K$. Existem uma subseqüência $\left(\mu_{n}^{\prime}\right)_{n \in \omega}$ de $\left(\mu_{n}\right)_{n \in \omega}$ e medidas $\nu_{n}$ e $\lambda_{n}$ tais que $\mu_{n}^{\prime}=\nu_{n}+\lambda_{n}$, $\left(\nu_{n}\right)_{n \in \omega}$ é duas a duas disjuntas e $\left(\lambda_{n}\right)_{n \in \omega}$ é fracamente convergente.

Demonstração: Tome $\mu=\sum_{n=0}^{\infty} \frac{\left|\mu_{n}\right|}{\| \mu_{n}|| 2^{n+1}}$. Temos que $\mu$ é uma medida positiva de norma 1.

Seja $i: L_{1}(\mu) \longrightarrow M(K)$ uma função dada por $i(h)(E)=\int_{E} h d \mu$, para todo $h \in L_{1}(\mu)$ e todo $E \subseteq K$ boreliano. Temos que $i$ é linear, pois, se $\lambda \in \mathbb{R}, g, h \in L_{1}(\mu)$ e $E \subseteq K$ é boreliano, temos

$$
i(\lambda g+h)(E)=\int_{E}(\lambda g+h) d \mu=\lambda \int_{E} g d \mu+\int_{E} h d \mu=\lambda i(g)+i(h) .
$$


Vejamos que $\|i(h)\|=\|h\|$, para todo $h \in L_{1}(\mu)$. Podemos escrever $h=$ $h_{+}-h_{-}$, onde $h_{+}$e $h_{-}$são funções borelianas positivas tais que $h_{+} \cdot h_{-}=0$. Sejam $E_{+}=h_{+}^{-1}((0, \infty))$ e $E_{-}=h_{-}^{-1}((0, \infty))$. Temos

$$
\begin{gathered}
\|i(h)\|=\left\|i\left(h_{+}\right)\right\|+\left\|i\left(h_{-}\right)\right\|=\int_{E_{+}} h_{+} d \mu+\int_{E_{-}} h_{-} d \mu= \\
\int_{K} h_{+}+h_{-} d \mu=\int|h| d \mu=\|h\| .
\end{gathered}
$$

Portanto $i$ é uma isometria sobre a imagem.

Seja $Y=i\left(L_{1}(\mu)\right)$. Mostraremos que $\mu_{n} \in Y$, para todo $n \in \omega$. Seja $n \in \omega$. Temos que $\mu_{n}$ é absolutamente contínua com respeito a $\mu$, isto é, se $\mu(E)=0$ então $\mu_{n}(E)=0$ (Definição 6.7 de $[\mathrm{Ru}]$ ). Logo, pelo Teorema de Radon-Nikodym (Teorema 6.10 de $[\mathrm{Ru}]$ ), para cada $n \in \omega$ existe um único $h_{n} \in L^{1}(\mu)$ tal que

$$
\mu_{n}(E)=\int_{E} h_{n} d \mu
$$

para todo $E$ mensurável, e teremos $i\left(h_{n}\right)=\mu_{n}$, provando que $\mu_{n} \in Y$.

Vejamos que $i$ leva seqüências duas a duas disjuntas em $L_{1}(\mu)$ em seqüências de medidas duas a duas disjuntas. Sejam $h_{1}, h_{2} \in L_{1}(\mu)$ tais que $h_{1}^{-1}(\mathbb{R} \backslash\{0\})$ e $h_{2}^{-1}(\mathbb{R} \backslash\{0\})$ são disjuntos. Sejam $E_{1}=h_{1}^{-1}(\mathbb{R} \backslash\{0\})$ e $E_{2}=h_{2}^{-1}(\mathbb{R} \backslash$ $\{0\})$. Temos $\left|i\left(h_{1}\right)\right|\left(K \backslash E_{1}\right)=\int_{K \backslash E_{1}}\left|h_{1}\right| d \mu=0$ e $\left|i\left(h_{2}\right)\right|\left(K \backslash E_{2}\right)=$ $\int_{K \backslash E_{2}}\left|h_{2}\right| d \mu=0$, provando que $i\left(h_{1}\right)$ e $i\left(h_{2}\right)$ são medidas disjuntas.

Falta mostrar que, se $\left(h_{n}\right)_{n \in \omega}$ é uma seqüência fracamente convergente em $L^{1}(\mu)$ então $\left(i\left(h_{n}\right)\right)_{n \in \omega}$ é fracamente convergente em $M(K)$. Como $i$ é um isomorfismo sabemos que $\left(i\left(h_{n}\right)\right)_{n \in \omega}$ é fracamente convergente em $Y$. Isso significa que existe $\mu \in M(K)$ tal que, para todo funcional linear $\varphi \in$ $Y^{*}, \varphi\left(i\left(h_{n}\right)\right)$ converge para $\varphi(\mu)$ (veja [Fa]). Logo, se $\psi \in M(K)^{*}$, temos que $\left.\psi\right|_{Y} \in Y^{*}$ e, portanto, $\psi\left(i\left(h_{n}\right)\right)=\left.\psi\right|_{Y}\left(i\left(h_{n}\right)\right)$ converge fracamente para $\left.\psi\right|_{Y}(i(h))=\psi(i(h))$

Portanto, aplicando a Proposição 4.5 para $\left(i^{-1}\left(\mu_{n}\right)\right)_{n \in \omega}$ concluímos o corolário.

\subsection{Construção de um forcing}

Nesta seção construímos o forcing que será usado para mostrar o Teorema 4.14. Essa construção baseia-se em [Ko1]. 
54 Um espaço $C(K)$ de densidade $\omega_{1}<2^{\omega}$ com poucos operadores

Seja $A \subseteq \mathcal{P}(\omega)$ uma álgebra de Boole enumerável. Seja $\mu$ uma medida de Radon positiva sobre $S(A)$, e seja $\varepsilon>0$, racional. Definimos um forcing $P(A, \mu, \varepsilon)=\left\{p: p=\left(p_{-1}, p_{1}\right) \in A^{2}, p_{-1} \cap p_{1}=\emptyset, p_{-1} \cup p_{1} \neq \omega, \mu\left(\left(p_{-1} \cup\right.\right.\right.$ $\left.\left.\left.p_{1}\right)^{*}\right)<\varepsilon\right\}$, com a ordem

$$
\left(p_{-1}, p_{1}\right) \leq\left(q_{-1}, q_{1}\right) \text { se, e somente se, } p_{-1} \supseteq q_{-1}, p_{1} \supseteq q_{1} \text {. }
$$

Definimos o forcing $R(A)$ formado pelas condições $p=\left(p_{-1}, p_{1} ; \mu_{1}, \ldots, \mu_{n} ; \varepsilon\right)$ tais que

- $\mu_{i} \in M(S(A))$ são medidas positivas tais que $\left\|\mu_{i}\right\| \geq \varepsilon$;

- $\varepsilon \in \mathbb{Q}, \varepsilon>0$;

- $\left(p_{-1}, p_{1}\right) \in \bigcap_{1 \leq n} P\left(A, \mu_{i}, \varepsilon\right)$.

Definimos a ordem $\leq$ por $\left(p_{-1}, p_{1} ; \mu_{1}, \ldots, \mu_{n} ; \varepsilon\right) \leq\left(q_{-1}, q_{1} ; \nu_{1}, \ldots, \nu_{m} ; \delta\right)$ se, e somente se, $p_{-1} \supseteq q_{-1}, p_{1} \supseteq q_{1},\left\{\mu_{1}, \ldots, \mu_{n}\right\} \supseteq\left\{\nu_{1}, \ldots, \nu_{m}\right\}, \varepsilon \leq \delta$.

Lema 4.7. Sejam $\varepsilon>0, \mu \in M(S(A))$ e $p=\left(p_{-1}, p_{1} ; \mu, \nu_{1}, \ldots, \nu_{m}, \varepsilon^{\prime}\right) \in$ $R(A)$ tal que $\varepsilon^{\prime} \leq \varepsilon$. Sejam $\mu_{n} \in M(S(A))$ e $\left(x_{n}\right)_{n \in \omega}$ uma seqüência em $S(A)$ tais que $\left\{\mu_{n}: n \in \omega\right\}$ não é $5 \varepsilon$-fracamente relativamente compacto, $\left|\mu_{n}\right|$ converge fracamente ${ }^{*}$ para $\mu, \mu_{n}\left(\left\{x_{n}\right\}\right) \stackrel{n}{\longrightarrow} 0$ e existe uma seqüência $\left(a_{n}\right)_{n \in \omega} \subseteq A$ duas a duas disjuntas tal que ||$\mu_{n}||-\left|\mu_{n}\right|\left(a_{n}^{*}\right)<\frac{\varepsilon}{18}|| \mu_{n}||$, para todo $n \in \omega$. Então existem $\delta_{1}>\delta_{2}>0$ tais que, para todos $k \in \omega$ e $r \leq p$, existem $q \leq r$ e $n_{1}, n_{2}>k$ tais que

1. $\left|\mu_{n_{1}}\left(q_{-1}^{*}\right)\right|>\delta_{1}$;

2. $\left|\mu_{n_{2}}\left(q_{-1}^{*}\right)\right|<\delta_{2}$;

3. $\left|\mu_{n_{1}}\right|\left(\left(-\left(q_{-1} \cup q_{1}\right)\right)^{*}\right)<\frac{\delta_{1}-\delta_{2}}{3}$;

4. $\left|\mu_{n_{2}}\right|\left(\left(-\left(q_{-1} \cup q_{1}\right)\right)^{*}\right)<\frac{\delta_{1}-\delta_{2}}{3}$;

5. $q_{-1} \in x_{n_{1}} \cap x_{n_{2}}$ ou $q_{1} \in x_{n_{1}} \cap x_{n_{2}}$. 
Demonstração: Pela Definição 4.1 podemos assumir que existe uma seqüência de abertos dois a dois disjuntos $\left(W_{n}\right)_{n \in \omega}$ tal que $\left|\mu_{n}\left(W_{n}\right)\right|>5 \varepsilon$, para todo $n \in \omega$.

Como $\left(\mu_{n}\right)_{n \in \omega}$ não é fracamente convergente (pelo Lema 4.2) temos que $\left\|\mu_{n}\right\|$ não converge a 0 . Passando a uma subseqüência assumimos que $\left\|\mu_{n}\right\|$ converge a $r>0$.

Tomamos $\delta_{1}=\frac{5 \varepsilon}{3} r$ e $\delta_{2}=\frac{3 \varepsilon}{2} r$. Para simplificar a notação, assumiremos que $r=1$, substituindo $\mu_{n}$ por $\frac{\mu_{n}}{r}$, para cada $n$, e $\mu$ por $\frac{\mu}{r}$. Passando a uma subseqüência e usando a hipótese, assumimos que $\left|\mu_{n}\right|\left(a_{n}^{*}\right)>1-\frac{\varepsilon}{18}$, para todo $n$.

Fixe um $\delta>0$ tal que $\delta<\frac{\varepsilon^{\prime}-\nu\left(\left(p_{-1} \cup p_{1}\right)^{*}\right)}{6}$, para todo $\nu \in\left\{\mu, \nu_{1}, \ldots, \nu_{m}\right\}$. Os valores de $\varepsilon^{\prime}-\nu\left(\left(p_{-1} \cup p_{1}\right)^{*}\right)$ são positivos pela definição do forcing. Usando o Lema de Rosenthal e a hipótese de que $\mu_{n}\left(\left\{x_{n}\right\}\right)$ converge a 0 , passando a uma subseqüência, assumimos que $\left|\mu_{n}\left(\left\{x_{m}\right\}\right)\right|<\delta$, para todos $n, m \in \omega$.

Fixemos $k \in \omega$ e $r \leq p$. Assumimos que $r=p$. Para $r<p$ o procedimento é idêntico.

Como $\left|\mu_{n}\right|$ converge fracamente* para $\mu$ e $\mu\left(\left(p_{-1} \cup p_{1}\right)^{*}\right)<\varepsilon$, tomamos $k_{0} \geq k$ tal que, para todo $n \geq k_{0}$,

$$
\left|\mu_{n}\right|\left(\left(p_{-1} \cup p_{1}\right)^{*}\right)<\varepsilon,
$$

de onde também segue que

$$
\left|\mu_{n}\left(p_{-1}^{*}\right)\right|<\varepsilon
$$

$\mathrm{e}$

$$
\left|\mu_{n}\left(p_{1}^{*}\right)\right|<\varepsilon
$$

Tomando $U_{n}^{\prime}=W_{n} \backslash\left(p_{-1} \cup p_{1}\right)^{*}$ temos, para todo $n>k_{0}$,

$$
\left|\mu_{n}\left(U_{n}^{\prime}\right)\right|>5 \varepsilon-\varepsilon=4 \varepsilon .
$$

Mas

$$
\left|\mu_{n}\right|\left(K \backslash a_{n}^{*}\right)<\frac{\varepsilon}{18}
$$

de onde segue que $\left|\mu_{n}\left(U_{n}^{\prime} \cap a_{n}^{*}\right)\right|>3 \varepsilon$. Sejam $U_{n}=U_{n}^{\prime} \cap a_{n}^{*}$. Pela regularidade das medidas e compacidade de $S(A)$, podemos assumir que $U_{n}=b_{n}^{*}$, onde $b_{n} \in A$ e $b_{n} \leq a_{n}$.

Como $a_{n}$ 's são dois a dois disjuntos, podemos tomar $k_{1} \geq k_{0}$ tal que, para todo $n>k_{1}, \mu\left(a_{n}^{*}\right)<\delta$ e $\nu_{i}\left(a_{n}^{*}\right)<\delta$, para todo $i=1, \ldots, m$. Assim 
teremos que, dados $n, j>k_{1}, \nu\left(\left(p_{-1} \cup p_{1} \cup a_{n} \cup a_{j}\right)^{*}\right)<\varepsilon^{\prime}-2 \delta$, para todo $\nu \in\left\{\mu, \nu_{1}, \ldots, \nu_{m}\right\}$.

Iremos cuidar do item 5 . Se $p_{1} \notin x_{n}$, para infinitos $n$ 's, passando a uma subseqüência assumimos que isso ocorre para todo $n$. Neste caso, para cada $n$ fixe $c_{n} \in x_{n}$ disjunto de $p_{1}$ tal que $\left|\mu_{m}\right|\left(c_{n}^{*}\right)<\delta$, para todo $m$ (o que é possível, pois $\left.\left|\mu_{m}\left(\left\{x_{n}\right\}\right)\right|<\delta\right)$. Se $p_{1} \in x_{n}$, para quase todos $n$ 's, passando a uma subseqüência assumimos que $p_{1} \in x_{n}$ para todo $n$, e teremos item 5 automaticamente satisfeito. Nesse caso, tomamos $c_{n}=0$.

Sejam $n_{1}, n_{2}>k_{1}$ dois inteiros distintos. Defina

$$
\begin{gathered}
q_{-1}=p_{-1} \cup b_{n_{1}} \cup c_{n_{1}} \cup c_{n_{2}}, \\
q_{1}=p_{1} \cup\left(a_{n_{1}}-q_{-1}\right) \cup\left(a_{n_{2}}-q_{-1}\right) .
\end{gathered}
$$

Pela escolha de $k_{1}$ acima, temos que $q=\left\{q_{-1}, q_{1} ; \mu, \nu_{1}, \ldots, \nu_{m}, \varepsilon\right\} \in R(A)$ e claramente $q \leq p$. Temos

$$
\left|\mu_{n_{1}}\left(q_{-1}^{*}\right)\right| \geq\left|\mu_{n_{1}}\left(b_{n_{1}}^{*}\right)\right|-\left|\mu_{n_{1}}\right|\left(p_{-1}^{*}\right)-\left|\mu_{n_{1}}\right|\left(\left(c_{n_{1}} \cup c_{n_{2}}\right)^{*}\right)>3 \varepsilon-\varepsilon-2 \delta \geq \frac{5 \varepsilon}{3}
$$

e

$$
\left|\mu_{n_{2}}\left(q_{-1}^{*}\right)\right| \leq\left|\mu_{n_{2}}\right|\left(b_{n_{1}}^{*}\right)+\left|\mu_{n_{2}}\right|\left(p_{-1}^{*}\right)+\left|\mu_{n_{2}}\right|\left(c_{n_{1}} \cup c_{n_{2}}\right)^{*}<\frac{\varepsilon}{18}+\varepsilon+2 \delta<\frac{3 \varepsilon}{2} .
$$

Como $a_{n_{1}} \cup a_{n_{2}} \subseteq q_{-1} \cup q_{1}$, para $n \in\left\{n_{1}, n_{2}\right\}$ concluímos que

$$
\left|\mu_{n}\right|\left(\left(-\left(q_{-1} \cup q_{1}\right)\right)^{*}\right) \leq \frac{\varepsilon}{18}=\frac{\delta_{1}-\delta_{2}}{3},
$$

concluindos os itens 1 a 4 . Item 5 segue de que $c_{n_{1}} \cup c_{n_{2}} \leq q_{-1}$, no caso em que $c_{n} \in x_{n}$, para todo $n$, ou $q_{1} \in c_{n}$, para todo $n$.

\subsection{Construção consistente de um espaço $C(K)$ de densidade menor que contínuo com poucos operadores}

Usando uma iteração de forcing da forma $R(A)$ como na seção anterior, nesta seção iremos construir consistentemente um espaço $C(K)$ com poucos operadores e densidade menor que contínuo. 
Seja $G$ um $R(A)$-genérico sobre o modelo inicial $M$. Em $M[G]$ seja $g=$ $\bigcup\left\{p_{-1}: \exists p_{1} \in A \exists \mu_{1}, \ldots, \mu_{n} \in M(S(A)) \exists \varepsilon>0\left(\left(p_{-1}, p_{1} ; \mu_{1}, \ldots, \mu_{n} ; \varepsilon\right) \in G\right)\right\}$. Defina $A_{G}$ a álgebra gerada por $A \cup\{g\}$ e seja $\dot{A}_{G}$ um $R(A)$-nome para $A_{G}$.

Considere $P$ a iteração com suportes finitos $\left(P_{\alpha}\right)_{\alpha<\omega_{1}}$ definidos por $P_{\alpha} \Vdash$ $\dot{Q}_{\alpha}=R\left(A_{\alpha}\right)$, onde $A_{0}$ é a álgebra dos subconjuntos finitos e os cofinitos de $\omega, A_{\alpha}=\bigcup_{\beta<\alpha} A_{\beta}$, se $\alpha$ é um ordinal limite, e $A_{\alpha+1}$ é a álgebra gerada por $A_{\alpha} \cup\left\{g_{\alpha}\right\}$, onde e $G_{\{\alpha\}}$ é um $R\left(A_{\alpha}\right)$-genérico sobre $M\left[G_{\alpha}\right]$ e $g_{\alpha}$ é definido por

$\bigcup\left\{p_{-1}: \exists p_{1} \in A \exists \mu_{1}, \ldots, \mu_{n} \in M\left(S\left(A_{\alpha}\right)\right) \exists \varepsilon>0\left(\left(p_{-1}, p_{1} ; \mu_{1}, \ldots, \mu_{n} ; \varepsilon\right) \in G_{\{\alpha\}}\right)\right\}$.

O modelo $M\left[G_{\alpha}\right]$ também será denotado por $M_{\alpha}$.

Lema 4.8. Para qualquer álgebra enumerável $A$ em $\mathcal{P}(\omega)$ o forcing $R(A)$ é c.c.c. e, portanto, o forcing $P$ é c.c.c.

Demonstração: Como $A \times A \times Q$ é enumerável, numa família não-enumerável de condições de $R(A)$ existem $p^{\prime}$ e $p^{\prime \prime}$ distintos tais que $p^{\prime}=\left(p_{-1}, p_{1} ; \mu_{1}, \ldots, \mu_{n} ; \varepsilon\right)$ e $p^{\prime \prime}=\left(p_{-1}, p_{1} ; \nu_{1}, \ldots, \nu_{m} ; \varepsilon\right)$. Tomando $q=\left(p_{-1}, p_{1} ; \mu_{1}, \ldots, \mu_{n}, \nu_{1}, \ldots \nu_{m} ; \varepsilon\right)$ temos que $q \in R(A)$ e $q \leq p^{\prime}, p^{\prime \prime}$.

Por ser uma iteração com suportes finitos de forcings c.c.c., $P$ também é c.c.c.

$\operatorname{Em} M[G]$ definimos $A_{\omega_{1}}=\bigcup_{\alpha<\omega_{1}} A_{\alpha}$.

Lema 4.9. Sejam $\dot{\mu}$ um P-nome para uma medida em $A_{\omega_{1}}$ e seja $\dot{z}$ um Pnome para um ponto de $S\left(A_{\omega_{1}}\right)$. Então os seguintes conjuntos são fechados ilimitados em $\omega_{1}$.

a) $C_{\dot{\mu}}=\left\{\alpha<\omega_{1}:\left.P \Vdash \dot{\mu}\right|_{\dot{A}_{\alpha}} \in M_{\alpha} e|\dot{\mu}|_{\mid \dot{A}_{\alpha}}=\left|\dot{\mu}_{\mid \dot{A}_{\alpha}}\right|\right\}$;

b) $C_{\dot{z}}=\left\{\alpha<\omega_{1}: P \Vdash \dot{z} \cap \dot{A}_{\alpha} \in M_{\alpha}\right\}$

Demonstração: Tome $\alpha_{0} \in \omega_{1}$. Construiremos, por indução, uma seqüência crescente $\left(\alpha_{n}\right)_{n \in \omega}$ em $\omega_{1}$ tal que $P$ força as seguintes sentenças:

$$
\left.\dot{\mu}\right|_{A_{\alpha_{n}}} \in M_{\alpha_{n+1}}
$$


e

$$
\forall a \in \dot{A}_{\alpha_{n}}\left(|\dot{\mu}|(a)=\left|\dot{\mu}_{\mid A_{\alpha_{n+1}}}\right|(a)\right) .
$$

Trabalhando em $M[G]$, para $G$ um $P$-genérico sobre um modelo inicial $M$, dado $n$, encontramos $\alpha_{n+1}>\alpha_{n}$ tal que $\dot{\mu}_{G} \in M_{\alpha_{n}}$, pois $A_{\alpha_{n}} \in M\left[G_{\alpha}\right]$ é enumerável e $\left.\dot{\mu}_{G}\right|_{A_{\alpha_{n}}}$ pode ser identificado como uma seqüência enumerável de seqüências enumeráveis de naturais. Da mesma forma, podemos tomar $\alpha_{n+1}$ suficientemente grande para conter todos os elementos de $A_{\omega_{1}}$ que decidem o valor de $\left|\dot{\mu}_{G}\right|(a)$, para $a \in A_{\alpha}$, uma vez que $\left|\dot{\mu}_{G}\right|(a)$ é o supremo de somas finitas de medidas de borelianos disjuntos, e cada boreliano em $S\left(A_{\omega_{1}}\right)$ pode ser aproximado por abertos-fechados.

Portanto, para cada $p \in P$ achamos $q \leq p$ que força $(*)$ e $(* *)$, para algum $\alpha_{n+1}$. Isto é, o conjunto $D$ formado pelas condições $p \in P$ tais que, para algum $\beta<\omega_{1}$,

$$
p \Vdash \beta=\min \left\{\beta<\omega_{1}:\left.\dot{\mu}\right|_{A_{\alpha_{n}}} \in M_{\beta} \wedge \forall a \in \dot{A}_{\alpha_{n}}\left(|\dot{\mu}|(a)=\left|\dot{\mu}_{\mid \beta}\right|(a)\right)\right\},
$$

é denso em $P$. Para cada $p \in D$ definimos $\beta^{p}$ como o menor $\beta$ tal que $p$ força $(*)$ e $(* *)$ para $\beta$ no lugar de $\alpha_{n+1}$. Observe que, se $\beta^{p} \neq \beta^{q}$ então $p$ e $q$ são incompatíveis. Como $P$ é c.c.c. temos que $\left\{\beta^{p}: p \in D\right\}$ é enumerável. Como $D$ é denso em $P$, tomando $\alpha_{n+1}=\sup \left\{\beta^{p}: p \in D\right\}$ teremos $(*)$ e $(* *)$ satisfeitos.

Tomando $\alpha=\sup \left\{\alpha_{n}: n \in \omega\right\}$, temos que $\alpha \in C_{\dot{\mu}}$, provando que $C_{\dot{\mu}}$ não é limitado. Para mostrar que $C_{\dot{\mu}}$ é fechado o argumento é análogo. Com isso concluímos item $a$ ). O item $b$ ) é análogo.

Lema 4.10. Sejam $\alpha<\omega_{1}, \varepsilon>0$ racional e $p \in P$ tais que $p(\alpha)=$ $\left(\dot{p}_{-1}^{\alpha}, \dot{p}_{1}^{\alpha} ; \dot{\mu}, \dot{\nu}_{1}, \ldots, \dot{\nu}_{m} ; \check{\varepsilon}\right)$. Sejam $\left(\dot{\mu}_{n}\right)_{n \in \omega}$ e $\left(\dot{x}_{n}\right)_{n \in \omega}$ seqüências de P-nomes tais que $p$ força:

1. $\dot{\mu}_{n} \in M\left(S\left(\dot{A}_{\omega_{1}}\right)\right)$;

2. $\dot{x}_{n} \in S\left(\dot{A}_{\omega_{1}}\right)$;

3. $\left.\mu_{n}\right|_{\dot{A}_{\alpha}} \in M_{\alpha}$;

4. $\dot{x}_{n} \cap \dot{A}_{\alpha} \in M_{\alpha}$;

5. $\left|\dot{\mu}_{n \mid \dot{A}_{\alpha}}\right|=\left|\dot{\mu}_{n}\right|_{\mid \dot{A}_{\alpha}} ;$ 
6. $\left.\dot{\mu}_{n}\right|_{\dot{A}_{\alpha}}\left(\left\{\dot{x}_{n} \cap \dot{A}_{\alpha}\right\}\right) \stackrel{n}{\longrightarrow} 0$;

7. $\left|\dot{\mu}_{n \mid \dot{A}_{\alpha}}\right|$ converge fracamente ${ }^{*}$ para $\dot{\mu}$;

8. $\left\{\left.\dot{\mu}_{n}\right|_{\dot{A}_{\alpha}}: n \in \check{\omega}\right\}$ não é $\check{5} \check{\varepsilon}$-fracamente relativamente compacto;

9. existe $\left(a_{n}\right)_{n \in \omega} \subseteq \dot{A}_{\alpha}$ dois a dois disjuntos tal que $\| \dot{\mu}_{n}||-\left|\dot{\mu}_{n}\right|\left(a_{n}^{*}\right)<$ $\frac{\varepsilon}{18}\left\|\dot{\mu}_{n}\right\|$.

Então existem $\delta_{1}, \delta_{2}>0$ tais que $p \Vdash \forall k \in \check{\omega} \exists n_{1}, n_{2}>k\left|\dot{\mu}_{n_{1}}\left(\dot{g}_{\alpha}\right)\right|>$ $\check{\delta}_{1},\left|\dot{\mu}_{n_{2}}\left(\dot{g}_{\alpha}\right)\right|<\delta_{2}$ e $\dot{g}_{\alpha} \in \dot{x}_{n_{1}} \cap \dot{x}_{n_{2}}$ ou $-\dot{g}_{\alpha} \in \dot{x}_{n_{1}} \cap \dot{x}_{n_{2}}$.

Demonstração: Seja $G$ um $P$-genérico sobre um modelo inicial $M$ tal que $p \in G$. Trabalhemos em $M\left[G_{\alpha}\right]$. Sejam $\mu_{n}=\left(\dot{\mu}_{n}\right)_{G_{\alpha}}$ e $x_{n}=\left(\dot{x}_{n}\right)_{G_{\alpha}}$. Pelas hipóteses deste lema podemos aplicar o Lema 4.7, obtendo $\delta_{1}^{\prime}>\delta_{2}^{\prime}>0$ tais que para todo $q \leq p$ e todo $k \in \omega$, existem $n_{1}, n_{2}>k$ e $r \leq q$ tais que $\left|\mu_{n_{1} \mid A_{\alpha}}\left(r_{-1}^{*}\right)\right|>\delta_{1}^{\prime},\left|\mu_{n_{2} \mid A_{\alpha}}\left(r_{-1}^{*}\right)\right|<\delta_{2}^{\prime}$ e $\left|\mu_{n_{i} \mid A_{\alpha}}\right|\left(\left(-\left(r_{-1} \cup r_{1}\right)\right)^{*}\right)<\frac{\delta_{1}^{\prime}-\delta_{2}^{\prime}}{3}$, para $i=1,2$. Da genericidade de $G_{\alpha}$ concluímos que, para cada $k$, existem $n_{1}, n_{2}>k$ e $r \leq p$ como acima tais que $r_{-1} \subseteq g_{\alpha}$ e $g_{\alpha} \cap r_{1}=\emptyset$. Da hipótese 5 do lema temos $\left|\mu_{n_{i}}\right|\left(\left(-\left(r_{-1} \cup r_{1}\right)\right)^{*}\right)<\frac{\delta_{1}^{\prime}-\delta_{2}^{\prime}}{3}$, para $i=1,2$. Portanto, para todo $k \in \omega$, existem $n_{1}, n_{2}>k$ tais que

$$
\left|\mu_{n_{1}}\left(g_{\alpha}\right)\right|>\delta_{1}^{\prime}-\frac{\delta_{1}^{\prime}-\delta_{2}^{\prime}}{3}
$$

e

$$
\left|\mu_{n_{2}}\left(g_{\alpha}\right)\right|<\delta_{2}^{\prime}+\frac{\delta_{1}^{\prime}-\delta_{2}^{\prime}}{3} .
$$

Tomamos $\delta_{1}=\delta_{1}^{\prime}-\frac{\delta_{1}^{\prime}-\delta_{2}^{\prime}}{3}$ e $\delta_{2}=\delta_{2}^{\prime}+\frac{\delta_{1}^{\prime}-\delta_{2}^{\prime}}{3}$, e obtemos que $\delta_{1}>\delta_{2}>0$ satisfazem a tese do lema. A segunda parte do lema segue do item 5 do Lema 4.7, uma vez que $q_{-1} \leq g$ e $q_{1} \leq-g$.

Lema 4.11. Seja $\alpha<\omega_{1}$. Sejam $a, b \in M_{\alpha}$ subconjuntos disjuntos de $\omega$ tais que $\overline{\left\{n^{*} \cap A_{\alpha}: n \in a\right\}} \cap \overline{\left\{n^{*} \cap A_{\alpha}: n \in b\right\}} \neq \emptyset$, em $M_{\alpha}$, tomando os fechos em $S\left(A_{\alpha}\right)$. Então $\overline{\left\{n^{*} \cap A_{\omega_{1}}: n \in a\right\}} \cap \overline{\left\{n^{*} \cap A_{\omega_{1}}: n \in b\right\}} \neq \emptyset$, em $M[G]$, tomando os fechos em $S\left(A_{\omega_{1}}\right)$. 
Demonstração: Observamos que, se $\overline{\left\{n^{*} \cap A_{\beta}: n \in a\right\}} \cap \overline{\left\{n^{*} \cap A_{\beta}: n \in b\right\}}=$ $\emptyset$, em $M_{\beta}$, para $\beta$ um ordinal limite, existem $\bar{a}, \bar{b} \in A_{\beta}$ disjuntos tais que $a \subseteq \bar{a}$ e $b \subseteq \bar{b}$. Logo existe $\gamma<\beta$ em que a separação acontece. Portanto, para mostrar o lema, é suficiente mostrar que $\overline{\left\{n^{*} \cap A_{\alpha+1}: n \in a\right\}} \cap$ $\overline{\left\{x_{n} \cap A_{\alpha+1}: n \in b\right\}} \neq \emptyset$, e prosseguimos por indução. Para isso, mostraremos que, se $A$ é uma subálgebra enumerável de $\mathcal{P}(\omega)$ contendo os unitários de $\omega$, e $a, b \subseteq \omega$ são como na hipótese do lema, então

$$
R(A) \Vdash \overline{\left\{n^{*} \cap \dot{A}_{G}: n \in \check{a}\right\}} \cap \overline{\left\{n^{*} \cap \dot{A}_{G}: n \in \check{b}\right\}} \neq \emptyset, \text { em } S\left(\dot{A}_{G}\right) .
$$

Seja $p=\left(p_{-1}, p_{1} ; \mu_{1}, \ldots, \mu_{m} ; \varepsilon\right) \in R(A)$. Mostraremos que existe $q \leq p$ tal que $q \Vdash \overline{\left\{n^{*} \cap \dot{A}_{G}: n \in \check{a}\right\}} \cap \overline{\left\{n^{*} \cap \dot{A}_{G}: n \in \check{b}\right\}} \neq \emptyset$.

Das definições de convergência e do espaço de Stone de $A$ é fácil verificar que, para todo $M \subseteq \omega$ e todo $v \in S(A)$,

$$
n^{*} \cap A \stackrel{n \in M}{\longrightarrow} v \Leftrightarrow v=\{c \in A:|M \backslash c|<\infty\}
$$

Como $A$ é enumerável, $S(A)$ é metrizável. Logo, pela hipótese, existem $u \in S(A)$ e subconjuntos infinitos $a^{\prime} \subseteq a$ e $b^{\prime} \subseteq b$ tais que $n^{*} \cap A \stackrel{n \in a^{\prime}}{\longrightarrow} u$ e $n^{*} \cap A \stackrel{n \in b^{\prime}}{\longrightarrow} u$. Isto é, $u=\left\{c \in A: a^{\prime} \backslash c\right.$ é finito $\}=\left\{c \in A: b^{\prime} \backslash c\right.$ é finito $\}$.

Consideramos três casos. Se existe $q \leq p$ tal que $q_{-1} \in u$, temos que $a^{\prime} \backslash q_{-1}$ e $b^{\prime} \backslash q_{-1}$ são finitos. Logo

$$
q \Vdash\left|\check{a}^{\prime} \backslash \dot{g}\right|<\infty
$$

e, para todo $a_{1} \in u$ e $a_{2} \in A$,

$$
q \Vdash\left|\check{a}^{\prime} \backslash\left(\check{a}_{1} \dot{g} \cup \check{a}_{2}\right)\right| \leq\left|\left(\check{a}^{\prime} \backslash \check{a}_{1}\right) \cup\left(\check{a}^{\prime} \backslash \dot{g}\right)\right|<\infty
$$

de onde temos que

$$
q \Vdash n^{*} \cap \dot{A}_{G} \stackrel{n \in \check{a}^{\prime}}{\longrightarrow}<\check{u} \cup\{\dot{g}\}>,
$$

onde $<u \cup\{g\}>$ é o ultrafiltro em $A_{G}$ gerado por $u$ e $g$. Repetindo o argumento temos

$$
q \Vdash n^{*} \cap \dot{A}_{G} \stackrel{n \in \check{b}^{\prime}}{\longrightarrow}<\check{u} \cup\{\dot{g}\}>,
$$

concluindo que

$$
q \Vdash \overline{\left\{n^{*} \cap \dot{A}_{G}: n \in \check{a}\right\}} \cap \overline{\left\{n^{*} \cap \dot{A}_{G}: n \in \check{b}\right\}} \neq \emptyset .
$$


No segundo caso, existe $q \leq p$ tal que $q_{1} \in u$. Analogamente ao caso anterior mostramos que

$$
q \Vdash n^{*} \cap \dot{A}_{G} \stackrel{n \in \check{a ̆}^{\prime} \cup \check{b}^{\prime}}{\longrightarrow}<\check{u} \cup\{\dot{g}\}>.
$$

Suponhamos que não acontecem os casos anteriores. Seja $q \leq p$. Como $u \notin\left(q_{-1} \cup q_{1}\right)^{*}$, temos que $a^{\prime} \backslash\left(q_{-1} \cup q_{1}\right)$ e $b^{\prime} \backslash\left(q_{-1} \cup q_{1}\right)$ são infinitos. Usando isso e que $\mu_{i}\left(\left\{n^{*}\right\}\right) \stackrel{n \in \omega}{\longrightarrow} 0$, para todo $i \in\{1, \ldots, m\}$, para cada $k \in \omega$ fixamos $n_{0, k}, n_{1, k}>k$ tais que

1. $n_{0, k} \in a^{\prime}, n_{1, k} \in b^{\prime}$;

2. $n_{0, k}, n_{1, k} \notin\left(q_{-1} \cup q_{1}\right)^{*}$;

3. $\mu_{i}\left(\left\{n_{0, k}, n_{1, k}\right\}^{*}\right)<\frac{\varepsilon-\mu_{i}\left(\left(q-1 \cup q_{1}\right)^{*}\right)}{2^{k+1}}$, para todo $i \in\{1, \ldots, m\}$.

Tome $r=\left(q_{-1} \cup\left\{n_{i, k}:(i, k) \in\{0,1\} \times \omega\right\}, q_{1} ; \mu_{1}, \ldots, \mu_{m} ; \varepsilon\right)$. As condições 2 e 3 acima garantem que $r \in R(A)$. Tomando $a^{\prime \prime}=\left\{n_{0, k}: k \in \omega\right\}$ e $b^{\prime \prime}=\left\{n_{1, k}: k \in \omega\right\}$, repetimos os argumentos anteriores para mostrar que

$$
r \Vdash n^{*} \cap \dot{A}_{G} \stackrel{n \in \check{a}^{\prime \prime} \cup \check{b ̆}^{\prime \prime}}{\longrightarrow}<\check{u} \cup\{\dot{g}\}>
$$

isto é,

$$
r \Vdash \overline{\left\{n^{*} \cap \dot{A}_{G}: n \in \check{a}\right\}} \cap \overline{\left\{n^{*} \cap \dot{A}_{G}: n \in \check{b}\right\}} \neq \emptyset .
$$

Lema 4.12. Se $U, V$ são abertos em $S\left(A_{\omega_{1}}\right)$ tais que $\bar{U} \cap \bar{V} \neq \emptyset$, então $\bar{U} \cap \bar{V}$ não é unitário.

Demonstração: Sejam $\dot{U}$ e $\dot{V} P$-nomes para abertos disjuntos de $S\left(A_{\omega_{1}}\right)$ tais que

$$
P \Vdash \overline{\dot{U}} \cap \overline{\dot{V}} \neq \emptyset \text {. }
$$

Para $\alpha<\omega_{1}$, sejam $\dot{U}_{\alpha}$ e $\dot{V}_{\alpha} P$-nomes tais que

$$
P \Vdash \pi_{\alpha}[\dot{U}]=\dot{U}_{\alpha}, \pi_{\alpha}[\dot{V}]=\dot{V}_{\alpha}
$$

Em $M[G]$, como $A_{\omega_{1}} \subseteq \mathcal{P}(\omega), A_{\omega_{1}}$ é c.c.c. Logo, se $V$ é aberto de $S\left(A_{\omega_{1}}\right)$, existe $V^{\prime}$ união enumerável de abertos básicos tal que $\bar{V}=\overline{V^{\prime}}$. Assim, no 
modelo inicial podemos assumir que $(\dot{U})_{G}$ e $(\dot{V})_{G}$ são $P$-nomes para uniões enumeráveis de abertos básicos. Tomemos $\gamma<\omega_{1}$ e $p \in P$ tais que

$$
p \Vdash \forall \alpha \geq \gamma\left(\dot{U}=\pi_{\alpha}^{-1}\left[\dot{U}_{\alpha}\right] \text { e } \dot{V}=\pi_{\alpha}^{-1}\left[\dot{V}_{\alpha}\right]\right) .
$$

Seja $q \leq p$. Seja $\dot{z}$ um $P$-nome para um ponto de $S\left(A_{\omega_{1}}\right)$ tal que

$$
q \Vdash \dot{z} \in \overline{\dot{U}} \cap \overline{\dot{V}}
$$

Pelo Lema 4.9, existe $\alpha>\gamma, \operatorname{supp}(q)$ tal que $P \Vdash \dot{z} \cap \dot{A}_{\alpha} \in M_{\alpha}$. Tome $\dot{y}$ um $P$-nome tal que $P \Vdash \dot{y}=\dot{z} \cap \dot{A}_{\alpha}$. Como $P \Vdash "\left\{n^{*} \cap \dot{A}_{\omega_{1}}: n \in \omega\right\}$ é denso em $S\left(\dot{A}_{\omega_{1}}\right)$ ", existem $P$-nomes $\dot{a}$ e $\dot{b}$ para subconjuntos disjuntos de $\omega$ pertencentes a $M_{\alpha}$ tais que

$$
q \Vdash \forall n \in \dot{a}\left(n^{*} \in \dot{U}\right), \forall n \in \dot{b}\left(n^{*} \in \dot{V}\right), n^{*} \cap \dot{A}_{\alpha} \stackrel{n \in \dot{a}}{\longrightarrow} \dot{y}, n^{*} \cap \dot{A}_{\alpha} \stackrel{n \in \dot{b}}{\longrightarrow} \dot{y} .
$$

Seja $r \leq q$ definido por $r(\beta)=q(\beta)$, se $\beta \neq \alpha$, e $r(\alpha)=\left(0,0 ; \delta_{\dot{y}} ; 1\right)$.

Trabalhemos em $M_{\alpha}$. Sejam $a=\dot{a}_{G_{\alpha}}, b=\dot{b}_{G_{\alpha}}$ e $y=\dot{y}_{G_{\alpha}}$, para um $P$ genérico $G$. Em $R\left(A_{\alpha}\right)$, não existe $s \leq r(\alpha)$ tal que $s_{-1} \in z$ ou $s_{1} \in z$, pois teríamos $\left|\delta_{y}\right|\left(\left(s_{-1} \cup s_{1}\right)^{*}\right)=1$, contradizendo a condição do forcing. Assim, analogamente à demonstração do Lema 4.11, concluímos que existem $a^{\prime} \subseteq a$ e $b^{\prime} \subseteq b$ infinitos tais que

$$
r(\alpha) \Vdash n^{*} \cap \dot{A}_{\alpha+1} \stackrel{n \in \dot{a}^{\prime} \cup \dot{b}^{\prime}}{\longrightarrow}<\check{y} \cup\left\{\dot{g}_{\alpha}\right\}>
$$

e, repetindo o argumento em 4.11 mas obtendo $a^{\prime \prime}, b^{\prime \prime} \subseteq q_{1}$, mostramos que

$$
r(\alpha) \Vdash n^{*} \cap \dot{A}_{\alpha+1} \stackrel{n \in \dot{a}^{\prime \prime} \cup \dot{b}^{\prime \prime}}{\longrightarrow}<\check{y} \cup\left\{-\dot{g}_{\alpha}\right\}>,
$$

de onde temos

$$
r(\alpha) \Vdash<\check{y} \cup\left\{\dot{g}_{\alpha}\right\}>\in \overline{\left\{n^{*} \cap \dot{A}_{\alpha+1}: n \in \check{a}\right\}} \cap \overline{\left\{n^{*} \cap \dot{A}_{\alpha+1}: n \in \check{b}\right\}}
$$

e

$$
r(\alpha) \Vdash<\check{y} \cup\left\{-\dot{g}_{\alpha}\right\}>\in \overline{\left\{n^{*} \cap \dot{A}_{\alpha+1}: n \in \check{a}\right\}} \cap \overline{\left\{n^{*} \cap \dot{A}_{\alpha+1}: n \in \check{b}\right\}},
$$

concluindo que, no modelo inicial $M$,

$$
r \Vdash \pi_{\alpha+1}^{-1}\left[<\check{y} \cup\left\{\dot{g}_{\alpha}\right\}>\right] \subseteq \overline{\dot{U}} \cap \overline{\dot{V}}, \pi_{\alpha+1}^{-1}\left[<\check{y} \cup\left\{-\dot{g}_{\alpha}\right\}>\right] \subseteq \overline{\dot{U}} \cap \overline{\dot{V}},
$$

ou seja,

$$
r \Vdash|\overline{\dot{U}} \cap \overline{\dot{V}}| \geq 2
$$

Lema 4.13. Todo operador em $C\left(S\left(A_{\omega_{1}}\right)\right)$ é multiplicador fraco. 
Demonstração: Trabalhemos em $M[G]$. Denotaremos $S\left(A_{\omega_{1}}\right)$ por $K$. Podemos identificar cada medida como uma função de $A_{\omega_{1}}$ em $\mathbb{R}$.

Suponha que exista $T: C(K) \longrightarrow C(K)$ que não é multiplicador fraco. Seja $D=\left\{m^{*} \cap A_{\omega_{1}}: m \in \omega\right\}$. Temos que $D$ é denso em $K$. Pelo Lema 4.3 existem $x_{n} \in D$ distintos tais que, para toda $f: K \longrightarrow \mathbb{R}$ limitada, $T^{*}\left(\delta_{x_{n}}\right)-$ $f \delta_{x_{n}}$ formam um conjunto não relativamente fracamente compacto. Tome $f: K \longrightarrow \mathbb{R}$ definida por $f\left(x_{n}\right)=T^{*}\left(\delta_{x_{n}}\right)\left(\left\{x_{n}\right\}\right)$, para todo $n \in \omega$, e $f(x)=0$ nos demais pontos de $K$. Pelo Corolário 4.6, passando a uma subseqüência, podemos assumir que existem medidas $\left(\mu_{n}\right)_{n \in \omega}$ duas a duas disjuntas e uma seqüência de medidas $\left(\lambda_{n}\right)_{n \in \omega}$ fracamente convergente para $\lambda$ tais que $T^{*}\left(\delta_{x_{n}}\right)-f \delta_{x_{n}}=\mu_{n}+\lambda_{n}$.

Considere $C=\bigcap_{n \in \omega} C_{\dot{\mu}_{n}}$, onde $\dot{\mu}_{n}$ são $P$-nomes para $\mu_{n}$ e $C_{\dot{\mu}_{n}}$ é definido como em 4.9. Pelos Lemas 4.9 e $1.25 C$ é fechado ilimitado em $\omega_{1}$. Tome $\alpha \in C$.

Também podemos assumir que $\left\{\left.\mu_{n}\right|_{A_{\alpha}}: n \in \omega\right\}$ não é fracamente relativamente compacto, tomando $\alpha$ suficiente para conter uma seqüência $a_{n}^{\prime} \mathrm{em}$ $A_{\omega_{1}}$ tal que $\left|\mu_{n}\left(a_{n}^{\prime}\right)\right|>\varrho$, para algum $\varrho>0$. Do mesmo modo, usando a Proposição 4.4, assumimos que existe uma seqüência $\left(a_{n}\right)_{n \in \omega}$ de elementos dois a dois disjuntos de $A_{\alpha}$ tal que ||$\mu_{n}||-\left|\mu_{n}\right|\left(a_{n}^{*}\right)<\frac{\varrho}{90}$. Além disso, veremos que podemos escolher $\alpha$ de modo que

$$
\begin{gathered}
\left.T^{*}\left(\delta_{x_{n}}\right)\right|_{A_{\alpha}}\left(\left\{x_{n} \cap A_{\alpha}\right\}\right)=T^{*}\left(\delta_{x_{n}}\right)\left(\left\{x_{n}\right\}\right), \\
\left.f \delta_{x_{n}}\right|_{A_{\alpha}}\left(\left\{x_{n} \cap A_{\alpha}\right\}\right)=f \delta_{x_{n}}\left(\left\{x_{n}\right\}\right)
\end{gathered}
$$

e

$$
\left.\lambda_{n}\right|_{A_{\alpha}}\left(\left\{x_{n} \cap A_{\alpha}\right\}\right)=\lambda_{n}\left(\left\{x_{n}\right\}\right)
$$

Para isso, usando regularidade das medidas, tomamos $a_{n, m}, b_{n, m}, c_{n, m} \in x_{n}$ tais que

$$
\begin{gathered}
\left|T^{*}\left(\delta_{x_{n}}\right)\left(a_{n, m}^{*}\right)-T^{*}\left(\delta_{x_{n}}\right)\left(\left\{x_{n}\right\}\right)\right|<\frac{1}{m}, \\
\left|f \delta_{x_{n}}\left(b_{n, m}^{*}\right)-f \delta_{x_{n}}\left(\left\{x_{n}\right\}\right)\right|<\frac{1}{m}, \\
\left|\lambda_{n}\left(c_{n, m}^{*}\right)-\lambda_{n}\left(\left\{x_{n}\right\}\right)\right|<\frac{1}{m}
\end{gathered}
$$

e tomamos $\alpha$ suficientemente grande para conter todos $a_{n, m}$ 's, $b_{n, m}$ 's e $c_{n, m}$ 's. Defina $z_{n}=x_{n} \cap A_{\alpha}$, para cada $n \in \omega$. 
Vejamos que $\left.\mu_{n}\right|_{A_{\alpha}}\left(\left\{x_{n} \cap A_{\alpha}\right\}\right) \stackrel{n}{\longrightarrow} 0$. De fato,

$$
\left.\mu_{n}\right|_{A_{\alpha}}\left(\left\{x_{n} \cap A_{\alpha}\right\}\right)=\mu_{n}\left(\left\{x_{n}\right\}\right)=T^{*}\left(\delta_{x_{n}}\right)\left\{x_{n}\right\}-f \delta_{x_{n}}\left(\left\{x_{n}\right\}\right)+\lambda_{n}\left(\left\{x_{n}\right\}\right) .
$$

Pelo Teorema de Dieudonné-Grothendieck, aplicado a vizinhanças de $x_{n}$ de medidas pequenas em relação a $\lambda_{n}$, temos que $\lambda_{n}\left(\left\{x_{n}\right\}\right) \stackrel{n}{\longrightarrow} 0$. Por outro lado temos

$$
\begin{aligned}
& T^{*}\left(\delta_{x_{n}}\right)\left\{x_{n}\right\}-f \delta_{x_{n}}\left(\left\{x_{n}\right\}\right)=T^{*}\left(\delta_{x_{n}}\right)\left\{x_{n}\right\}-\int_{\left\{x_{n}\right\}} f \delta_{x_{n}}= \\
& T^{*}\left(\delta_{x_{n}}\right)\left\{x_{n}\right\}-f\left(x_{n}\right)=T^{*}\left(\delta_{x_{n}}\right)\left\{x_{n}\right\}-T^{*}\left(\delta_{x_{n}}\right)\left\{x_{n}\right\}=0 .
\end{aligned}
$$

Trabalhando em $M_{\alpha}$, como $A_{\alpha}$ é enumerável, $S\left(A_{\alpha}\right)$ tem peso enumerável e, portanto, $C\left(S\left(A_{\alpha}\right)\right)$ é separável. Logo, $B_{X^{*}}$, a bola unitária de $X^{*}$, com a topologia fraca* é metrizável (Proposição 3.24 de [Fa]). Como, pelo Teorema de Alaoglu, $B_{X^{*}}$ é compacto na topologia fraca* (Teorema 3.21 de $[\mathrm{Fa}])$, podemos assumir, passando a uma subseqüência, que $\left|\mu_{\left.n\right|_{A_{\alpha}}}\right|$ converge fracamente*, em $M\left(S\left(A_{\alpha}\right)\right)$. Seja $\mu$ o limite fraco* de $\left|\mu_{\left.n\right|_{A_{\alpha}}}\right|$.

Sejam $\dot{\mu}_{n} P$-nomes para $\mu_{n}$ e $\dot{x}_{n} P$-nomes para $x_{n}$.

Passando a uma subseqüência assumimos que $\left(x_{n} \cap A_{\alpha}\right)_{n \in \omega}$ é uma seqüência convergente.

Seja $\varepsilon=\frac{\varrho}{5}$ Tome $q \in P$ definido por $q(\alpha)=(\emptyset, \emptyset, \mu, \varepsilon)$ e $q(\beta)=p(\beta)$ para $\beta \neq \alpha$.

Tome $r \leq q$ de modo a satisfazer as hipóteses do Lema 4.10. Usando o Lema 4.10 obtemos $\delta_{1}>\delta_{2}>0$ tais que

$$
r \Vdash \forall k \exists n_{1}, n_{2}>k\left|\dot{\mu}_{n_{1}}\left(g_{\alpha}\right)\right|>\delta_{1}>\delta_{2}>\left|\dot{\mu}_{n_{2}}\left(g_{\alpha}\right)\right|, g_{\alpha} \in \dot{x}_{n_{1}} \cap \dot{x}_{n_{2}}
$$

$\mathrm{Ou}$

$$
r \Vdash \forall k \exists n_{1}, n_{2}>k\left|\dot{\mu}_{n_{1}}(g)\right|>\delta_{1}>\delta_{2}>\left|\dot{\mu}_{n_{2}}\left(g_{\alpha}\right)\right|,-g_{\alpha} \in \dot{x}_{n_{1}} \cap \dot{x}_{n_{2}} .
$$

Encontramos, então, subconjuntos infinitos disjuntos $a_{\alpha}, b_{\alpha} \in M_{\alpha}\left[G_{\alpha}\right]$ de $\omega$ tais que $\left|\mu_{n}\left(g_{\alpha}\right)\right|>\delta_{1}$, para $n \in a_{\alpha}$, e $\left|\mu_{n}\left(g_{\alpha}\right)\right|<\delta_{2}$, para $n \in b_{\alpha}$, e, ou $g_{\alpha} \in x_{n}$, para todo $n \in a_{\alpha} \cup b_{\alpha}$, ou $-g_{\alpha} \in x_{n}$, para todo $n \in a_{\alpha} \cup b_{\alpha}$. Refinando $a_{\alpha}$ e $b_{\alpha}$, podemos assumir que existe $L \in \mathbb{R}$ tal que, para todo $n \in a_{\alpha} \cup b_{\alpha}$,

$$
\left|f\left(x_{n}\right)-\lambda\right|<\frac{\delta_{1}-\delta_{2}}{8}
$$


Como convergência fraca implica convergência fraca*, também assumimos que

$$
\left|\lambda_{n}\left(g_{\alpha}^{*}\right)-\lambda\left(g_{\alpha}^{*}\right)\right|<\frac{\delta_{1}-\delta_{2}}{8},
$$

para todo $n \in a_{\alpha} \cup b_{\alpha}$. Como $\left(x_{n} \cap A_{\alpha+1}\right)_{n \in a_{\alpha}}$ e $\left(x_{n} \cap A_{\alpha+1}\right)_{n \in b_{\alpha}}$ convergem a $<z \cup\left\{g_{\alpha}\right\}>$, onde $z$ é o limite de $\left(x_{n} \cap A_{\alpha}\right)_{n \in \omega}$, temos

$$
\overline{\left\{x_{n} \cap A_{\alpha+1}: n \in a_{\alpha}\right\}} \cap \overline{\left\{x_{n} \cap A_{\alpha+1}: n \in b_{\alpha}\right\}} \neq \emptyset
$$

em $S\left(A_{\alpha+1}\right)$. Como $a_{\alpha}, b_{\alpha} \in M_{\alpha}\left[G_{\alpha}\right]=M_{\alpha+1}$, pelo Lema 4.11 temos

$$
\overline{\left\{x_{n}: n \in a_{\alpha}\right\}} \cap \overline{\left\{x_{n}: n \in b_{\alpha}\right\}} \neq \emptyset,
$$

em $K$. Mas, usando que $T\left(\chi_{g_{\alpha}^{*}}\right)\left(x_{n}\right)=T^{*}\left(\left\{\delta_{x_{n}}\right\}\right)\left(g_{\alpha}^{*}\right)$ e $f \delta_{x_{n}}\left(\chi_{g_{\alpha}^{*}}\right)=f\left(x_{n}\right)$ temos

$T\left(\chi_{g_{\alpha}^{*}}\right)\left(x_{n}\right)=\mu_{n}\left(g_{\alpha}\right)+f\left(x_{n}\right)+\lambda_{n}\left(g_{\alpha}^{*}\right) \in\left\{x \in \mathbb{R}:\left|x-L-\lambda\left(g_{\alpha}^{*}\right)\right|>\delta_{1}-\frac{\delta_{1}-\delta_{2}}{4}\right\}$, para $n \in a_{\alpha} \mathrm{e}$

$T\left(\chi_{g_{\alpha}^{*}}\right)\left(x_{n}\right)=\mu_{n}\left(g_{\alpha}\right)+f\left(x_{n}\right)+\lambda_{n}\left(g_{\alpha}^{*}\right) \in\left\{x \in \mathbb{R}:\left|x-L-\lambda\left(g_{\alpha}^{*}\right)\right|<\delta_{2}+\frac{\delta_{1}-\delta_{2}}{4}\right\}$,

para $n \in b_{\alpha}$, de onde temos, pela continuidade de $T\left(\chi_{g_{\alpha}^{*}}\right)$, que $\overline{\left\{x_{n}: n \in a_{\alpha}\right\}}$ e $\overline{\left\{x_{n}: n \in b_{\alpha}\right\}}$ são disjuntos, chegando numa contradição.

Teorema 4.14. É consistente com ZFC que existe um espaço de Banach $C(K)$ de densidade $\omega_{1}<2^{\omega}$ tal que todo operador em $C(K)$ é da forma $g I+S$, onde $g \in C(K)$ e $S$ é fracamente compacto.

Demonstração: Suponha $\neg C H$ no modelo inicial $M$. Como $P$ é c.c.c. (Lema 4.8), $P$ preserva cardinais e, como $\left(2^{\omega}\right)^{M} \leq\left(2^{\omega}\right)^{M[G]}, \neg C H$ é preservado em $M[G]$. Por ser uma união de uma família de cardinalidade $\omega_{1}$ de álgebras enumeráveis, $A_{\omega_{1}}$ tem cardinalidade $\omega_{1}$ em $M[G]$ e, portanto, a densidade de $C(K)$ é igual ao peso de $K$, que é $\omega_{1}<2^{\omega}$. Dos Lema 4.13, 4.12 e 1.10 e do Teorema 1.11 segue que todo operador em $C(K)$ é da forma $g I+S$, como no enunciado. 
66 Um espaço $C(K)$ de densidade $\omega_{1}<2^{\omega}$ com poucos operadores 


\section{Capítulo 5}

\section{Um espaço $C(K)$ indecomponível de densidade}

$\omega_{1}<2^{\omega}$

Adaptando a construção do Capítulo 4 para o caso conexo, obtemos um espaço $C(K)$ indecomponível de densidade menor que contínuo. Como no Capítulo 4 , o resultado aqui obtido é independente de $\mathrm{ZFC}+\neg \mathrm{CH}$, uma vez que o axioma de Martin implica que todo $C(K)$ de densidade menor que contínuo não é de Grothendieck e, portanto, contém $c_{0}$ complementado.

Com isso respondemos parcialmente sobre as possíveis densidades de espaços de Banach $C(K)$ indecomponíveis, embora muito pouco ainda sabemos sobre isso. Vimos que $C(K)$ separável de dimensão infinita não pode ser indecomponível, pois teria $c_{0}$ complementado. Os espaços $C(K)$ indecomponíveis construídos em [Ko2] e [Pl] têm densidade $2^{\omega}$. Por resultados de [Fr] sabemos que é consistente, mesmo assumindo $\neg \mathrm{CH}$, que todo espaço $C(K)$ de densidade menor que contínuo é decomponível (vide introdução do Capítulo 4.) Mostramos, neste capítulo, que é consistente a existência de $C(K)$ indecomponível de densidade $\omega_{1}<2^{\omega}$.

Em [Ko3] foi mostrado que existe consistentemente $C(K)$ de densidade maior que contínuo com poucos operadores. Porém ainda não sabemos se existe $C(K)$ indecomponível de densidade maior que contínuo. Esse problema permanece em aberto mesmo para espaços de Banach em geral. Outro problema em aberto é se existe um limitante superior para a densidade de espaços de Banach indecomponíveis (da forma $C(K)$ ou geral). 


\subsection{Construção do forcing}

Sejam $\alpha<\omega_{1}$ e $K \subseteq[0,1]^{\alpha}$ compacto sem pontos isolados. Seja $\mathcal{B}_{\alpha}$ a base de abertos de $[0,1]^{\alpha}$ formada pelas uniões finitas de abertos elementares da forma $\Pi_{\beta<\alpha} I_{\beta}$, onde $I_{\beta}$ é um intervalo aberto, em $[0,1]$, de extremos racionais e $\left\{\beta<\alpha: I_{\beta} \neq[0,1]\right\}$ é finito. Notamos que $\mathcal{B}_{\alpha}$ é uma base de abertos de $[0,1]^{\alpha}$ fechada por uniões e intersecções finitas. Conforme vimos na Seção 1.1, uma medida em $[0,1]^{\alpha}$ pode ser representada por uma função de $\mathcal{B}_{\alpha}$ em $\mathbb{R}$. Uma medida em $K$ pode ser representada por uma medida $\mu$ em $[0,1]^{\alpha}$ tal que $|\mu|\left([0,1]^{\alpha} \backslash K\right)=0$.

Se $\alpha<\beta$, interpretamos $\mathcal{B}_{\alpha}$ como subconjunto de $\mathcal{B}_{\beta}$, identificando $V \in$ $\mathcal{B}_{\alpha} \operatorname{com} V \times[0,1]^{\beta \backslash \alpha} \in \mathcal{B}_{\beta}$. Assim, se $\mu$ é uma medida em $[0,1]^{\beta}$ e $\nu$ é uma medida $[0,1]^{\alpha}$, dizer que $\left.\mu\right|_{\mathcal{B}_{\alpha}}=\nu$ significa que $\mu\left(\pi_{\alpha}^{-1}[E]\right)=\nu(E)$, para todo $E \subseteq[0,1]^{\alpha}$ boreliano.

Definimos um forcing $R(K)$ formado pelas condições $p=\left(f_{p}, \Omega_{p}, M_{p}, \varepsilon_{p}, \Delta_{p}\right)$ tais que

A.1. $f_{p}: K \longrightarrow[0,1]$ contínua;

A.2. $\Omega_{p} \in \mathcal{B}_{\alpha} \backslash\left\{[0,1]^{\alpha}\right\}$

A.3. $\operatorname{supp}\left(f_{p}\right) \subseteq K \cap \Omega_{p}$;

A.4. $M_{p}$ é um conjunto finito de medidas positivas em $K$;

A.5. $\varepsilon_{p} \in \mathbb{Q} \cap(0, \infty)$;

A.6. $\mu\left(\bar{\Omega}_{p}\right)<\varepsilon_{p}$, para todo $\mu \in M_{p}$;

A.7. $\Delta_{p} \in \mathcal{B}_{\alpha+1}$

A.8. $\pi_{\alpha}\left[\bar{\Delta}_{p}\right]=\bar{\Omega}_{p}$;

A.9. $G r\left(\left.f_{p}\right|_{\bar{\Omega}_{p} \cap K}\right) \subseteq \bar{\Delta}_{p}$.

A ordem $\leq$ em $R(K)$ é dada por $q \leq p$ se, e somente se,

B.1. $\Omega_{q} \supseteq \Omega_{p}$;

B.2. $M_{q} \supseteq M_{p}$;

B.3. $\varepsilon_{q} \leq \varepsilon_{p}$; 
B.4. $\bar{\Delta}_{q} \cap\left(\bar{\Omega}_{p} \times[0,1]\right) \subseteq \bar{\Delta}_{p}$.

Dado $p \in R(K)$ definimos

$$
\operatorname{diam}\left(\Delta_{p}\right)=\sup \left\{\left|y_{1}-y_{2}\right|: \exists x \in \Omega_{p}\left(\left\{\left(x, y_{1}\right),\left(x, y_{2}\right)\right\} \subseteq \Delta_{p}\right)\right\},
$$

quando $\Delta_{p} \neq \emptyset$. Caso contrário convencionamos que $\operatorname{diam}\left(\Delta_{p}\right)=0$. É fácil verificar que, para toda condição $p \in R(K)$ e todos $q, r \leq p$, temos

$$
\forall x \in \bar{\Omega}_{p} \cap K\left|f_{q}(x)-f_{r}(x)\right| \leq \operatorname{diam}\left(\Delta_{p}\right) .
$$

Lema 5.1. Dados $p \in R(K)$ e $\varepsilon>0$ existe $q \leq p$ tal que $f_{q}=f_{p}, \Omega_{q}=\Omega_{p}$, $M_{q}=M_{p}, \varepsilon_{q}=\varepsilon_{p} e \operatorname{diam}\left(\Delta_{q}\right) \leq \varepsilon$.

Demonstração: Usando o Teorema de Tietze, encontramos $f:[0,1]^{\alpha} \longrightarrow$ $[0,1]$ que estende continuamente $f_{p}$. Seja $L$ o gráfico de $f$. Usando a continuidade de $f$, para cada $x \in L$ considere $V_{x} \in \mathcal{B}_{\alpha}$ um aberto elementar contendo $x$ tal que $|f(y)-f(x)|<\frac{\varepsilon}{8}$, para todo $y \in V_{x}$. Tome $I_{x}$ um intervalo aberto em $[0,1]$ de extremos racionais tal que

$$
\left(f(x)-\frac{\varepsilon}{8}, f(x)+\frac{\varepsilon}{8}\right) \cap[0,1] \subseteq I_{x} \subseteq\left(f(x)-\frac{\varepsilon}{4}, f(x)+\frac{\varepsilon}{4}\right) \cap[0,1] .
$$

Considere $W_{x}=V_{x} \times I_{x} \in \mathcal{B}_{\alpha+1}$. Por construção, $(y, f(y)) \in W_{x}$, para todo $y \in V_{x}$. Logo, $\left\{W_{x}: x \in[0,1]^{\alpha}\right\}$ é um recobrimento de $L$. Como $L$ é fechado em $[0,1]^{\alpha+1}$, por ser gráfico de uma função contínua, e, portanto, é compacto, podemos tomar $F \subseteq[0,1]^{\alpha}$ finito tal que $L \subseteq \cup\left\{W_{x}: x \in F\right\}$. Defina $\Delta_{q}=\Delta_{p} \cap\left(\cup\left\{W_{x}: x \in F\right\}\right)$. Como $L \subseteq \cup\left\{W_{x}: x \in F\right\}$, a condição A.9 do forcing é satisfeita. Pela construção de $\Delta_{q}$ temos $\Delta_{q} \in \mathcal{B}_{\alpha+1}$, satisfazendo A.7. De A.9 e do fato que $\Delta_{q} \subseteq \Delta_{p}$ seguem A.8 e B.4. Falta mostrarmos que $\operatorname{diam}\left(\Delta_{q}\right) \leq \varepsilon$.

Sejam $\left(x, y_{1}\right),\left(x, y_{2}\right) \in \Delta_{q}$. Sejam $x_{1}, x_{2} \in F$ tais que $\left(x, y_{1}\right) \in W_{x_{1}}$ e $\left(x, y_{2}\right) \in W_{x_{2}}$. Como $y_{1} \in I_{x_{1}}$ temos que $\left|y_{1}-f\left(x_{1}\right)\right|<\frac{\varepsilon}{4}$, pois $I_{x_{1}} \subseteq$ $\left(f\left(x_{1}\right)-\frac{\varepsilon}{4}, f\left(x_{1}\right)+\frac{\varepsilon}{4}\right)$. Como $x \in V_{x_{1}}$, temos $\left|f(x)-f\left(x_{1}\right)\right|<\frac{\varepsilon}{8}$. Logo

$$
\left|y_{1}-f(x)\right| \leq\left|y_{1}-f\left(x_{1}\right)\right|+\left|f\left(x_{1}\right)-f(x)\right| \leq \frac{\varepsilon}{4}+\frac{\varepsilon}{8}<\frac{\varepsilon}{2} .
$$

Analogamente concluímos que $\left|y_{2}-f(x)\right|<\frac{\varepsilon}{2}$ e, portanto, $\left|y_{1}-y_{2}\right|<\varepsilon$. 
Lema 5.2. Seja $p \in R(K)$, para $K \subseteq[0,1]^{\alpha}$ compacto. Sejam $\Omega \in \mathcal{B}_{\alpha}$ tal que $\Omega \supseteq \Omega_{p}, f: K \longrightarrow[0,1]$ contínua com suporte incluído em $\Omega \cap K$ tal que $\operatorname{Gr}\left(\left.f\right|_{\Omega_{p} \cap K}\right) \subseteq \Delta_{p}, M$ um conjunto finito de medidas positivas em $[0,1]^{\alpha}$ contendo $M_{p}$ e $\varepsilon \in \mathbb{Q} \cap\left(0, \varepsilon_{p}\right]$ tal que $\mu(\Omega)<\varepsilon$, para todo $\mu \in M$. Então existe $q \leq p$ tal que $f_{q}=f, \Omega_{q}=\Omega, M_{q}=M$ e $\varepsilon_{q}=\varepsilon$.

Demonstração: Pelas hipóteses do lema, tudo que precisamos é definir $\Delta_{q}$ de modo a satisfazer A.7, A.8, A.9 e B.4 da condição do forcing.

Observamos que, se $V \in \mathcal{B}_{\alpha}$, então $[0,1]^{\alpha} \backslash \bar{V} \in \mathcal{B}_{\alpha}$. De fato, considere $V=\Pi_{\beta<\alpha} I_{\beta}$ onde $I_{\beta_{0}}$ é um intervalo aberto em $[0,1]$ de extremos racionais, para um $\beta_{0}<\alpha$, e $I_{\beta}=[0,1]$, para todo $\beta \neq \beta_{0}$. Temos que $[0,1]^{\alpha} \backslash \bar{V}=$ $\Pi_{\beta<\alpha} J_{\beta}$, onde $J_{\beta}=[0,1]$, para $\beta \neq \beta_{0}$ e $J_{\beta_{0}}=[0,1] \backslash \bar{I}_{\beta_{0}}$, que é a união de dois intervalos abertos (possivelmente um deles, ou ambos, vazio) em $[0,1]$ também de extremos racionais. Portanto $[0,1]^{\alpha} \backslash \bar{V} \in \mathcal{B}_{\alpha}$. Para o caso geral, basta verificar que todo elemento de $\mathcal{B}_{\alpha}$ é uma união finita de intersecções finitas de abertos dessa forma. Como $W \backslash \bar{V}=W \cap\left([0,1]^{\alpha} \backslash \bar{V}\right)$, para $V, W \in \mathcal{B}_{\alpha}$, teremos também $W \backslash \bar{V} \in \mathcal{B}_{\alpha}$.

Portanto, $\Omega_{q} \backslash \bar{\Omega}_{p} \in \mathcal{B}_{\alpha}$. Para concluir o lema basta tomarmos $\Delta_{q}=$ $\left(\Omega_{q} \backslash \bar{\Omega}_{p}\right) \times[0,1] \cup \Delta_{p}$.

Lema 5.3. Sejam $\alpha<\omega_{1}$ e $K \subseteq[0,1]^{\alpha}$ compacto. Então

$\forall \varepsilon>0 \forall p \in R(K) \exists q \leq p \forall p_{1}, p_{2} \leq q \forall x \in \Omega_{q} \cap K\left|f_{p_{1}}(x)-f_{p_{2}}(x)\right|<\varepsilon$.

Demonstração: Sejam $\varepsilon>0$ e $p \in R(K)$. Pelo Lema 5.1 temos que $D_{\varepsilon}=\left\{q \in P: \operatorname{diam}\left(\Delta_{p}\right)<\varepsilon\right\}$ é denso em $R(K)$. Logo existe $q \leq p$ tal que $\operatorname{diam}\left(\Delta_{q}\right)<\varepsilon$. Por $(*)$, para todos $p_{1}, p_{2} \leq p$ e $x \in \Omega_{p} \cap K$ temos $\left|f_{p_{1}}(x)-f_{p_{2}}(x)\right|<\varepsilon$.

Seja $M$ um modelo transitivo enumerável para ZFC, com $R(K) \in M$, e seja $G$ um $R(K)$-genérico sobre $M$. Observamos que, como $\mathbb{R}^{M} \neq \mathbb{R}^{M[G] \text {, }}$ poderemos ter que $K$ não seja compacto em $M[G]$. Os abertos da base $\mathcal{B}_{\alpha}$ também podem mudar, mas usaremos a mesma notação para eles, pois os identificamos com as extremidades racionais correspondentes. Por exemplo, se $K \subseteq[0,1]^{2}$, se definirmos um aberto $V=\left(\frac{1}{2}, 1\right] \times[0,1] \in \mathcal{B}_{\alpha}$, usaremos a mesma notação $V$ tanto em $M$ quanto em $M[G]$, pois conjuntos finitos de racionais são absolutos. Mas, como adicionamos reais, teremos $V^{M} \neq V^{M[G]}$. Observamos que $V^{M[G]} \cap \bar{K}=V^{M} \cap K$. 
Seja $p \in R(K)$. Como $f_{p}$ é contínua em um compacto $K$, no modelo $M, f_{p}$ é uniformemente contínua em $M$ e, portanto, o é em $M[G]$, pois ser uniformemente contínua é uma fórmula absoluta em relação a modelos transitivos ${ }^{1}$. Portanto, em $M[G]$, podemos estender $f_{p}$ continuamente para uma função $\tilde{f}_{n}: \bar{K} \longrightarrow[0,1]$, definindo $\tilde{f}_{p}(x)=\lim _{n \in \omega} f_{p}\left(x_{n}\right)$, para $x_{n} \in K$ tal que $x_{n} \stackrel{n}{\longrightarrow} x$. Seja $\dot{f}_{p}$ um $R(K)$-nome para $\tilde{f}_{p}$.

Lembramos a definição de limite em um sistema dirigido: Se $F$ é um filtro em uma ordem parcial $P,\left(x_{p}\right)_{p \in F}$ é uma seqüência de reais indexada em $F$, e $x \in \mathbb{R}$, dizemos que $\lim _{p \in F} x_{n}=x$ se para todo $\varepsilon>0$ existe $p \in F$ tal que $\left|x_{q}-x\right|<\varepsilon$, para todo $q \leq p$. Como acontece com seqüências indexadas em $\omega$, é fácil verificar que um sistema dirigido $\left(x_{p}\right)_{p \in F}$ converge para algum $x \in \mathbb{R}$ se, e somente se, para todo $\varepsilon>0$ existe $p \in F$ tal que $\left|x_{q}-x_{r}\right|<\varepsilon$, para todos $q, r \leq p$.

$\operatorname{Em} M[G]$, defina $\Omega_{G}=\bigcup_{p \in G} \Omega_{p}$ e $f_{G}: \Omega_{G} \cap \bar{K} \longrightarrow[0,1]$ dada por

$$
f_{G}(x)=\lim _{p \in G} \tilde{f}_{p}(x) .
$$

A boa definição de $f_{G}$ segue do Lema 5.3 e da genericidade de $G$. Seja $K_{G}$ o fecho do gráfico de $f_{G}$. Iremos denotar por $\dot{f}_{G}$ e $\dot{K}_{G}$ os $R(K)$-nomes para $f_{G}$ e $K_{G}$, respectivamente.

Lema 5.4. Seja $K \subseteq[0,1]^{\alpha}$ um compacto sem pontos isolados, no modelo inicial $M$, e seja $G$ um $R(K)$-genérico sobre $M$. Então, em $M[G]$ temos:

(a) $\Omega_{G} \cap \bar{K}$ é denso em $\bar{K}$;

(b) $f_{G}$ é contínua;

(c) Se $K$ é conexo em $M, K_{G}$ é conexo em $M[G]$.

Demonstração: $\operatorname{Em} M$, mostraremos que, dados $x \in K, V$ uma vizinhança aberta de $x$ pertencente a $\mathcal{B}_{\alpha}$ e $p \in P$, existe $q \leq p$ tal que $V \cap \Omega_{q} \neq \emptyset$. Isso será suficiente para concluir $(a)$, usando, em $M[G]$, a genericidade de $G$ e que $K$ é denso em $\bar{K}$.

\footnotetext{
${ }^{1}$ Podemos escrever a fórmula da continuidade uniforme como $\forall n \in \omega \backslash\{0\} \exists m \in$ $\omega \backslash\{0\} \forall x, y \in K\left(|x-y|<\frac{1}{n} \rightarrow|f(x)-f(y)|<\frac{1}{m}\right)$, que é logicamente equivalente a $\neg \exists n\left(n \in \omega \backslash\{0\} \wedge \neg \exists m\left(m \in \omega \backslash\{0\} \wedge \neg \exists x\left(x \in K \wedge \exists y\left(y \in K \wedge\left(|x-y|<\frac{1}{m} \wedge|f(x)-f(y)| \geq\right.\right.\right.\right.\right.$ $\left.\left.\left.\left.\frac{1}{n}\right)\right)\right)\right)$ ). Como $f$ e $K$ pertencem ao modelo inicial e $\omega$ é absoluto, essa fórmula é $\Delta_{0}$ e, portanto, absoluta em relação a $M$ e $M[G]$ (vide Seção B.5).
} 
Se $x \in \bar{\Omega}_{p}$, tome $q$ como $p$ exceto que $\Omega_{q} \supseteq \bar{\Omega}_{p}$ preservando a condição $\mu\left(\Omega_{q}\right)<\varepsilon_{p}$, para todo $\mu \in M_{p}$ (é possível pela regularidade de $\mu$ ), e usamos o Lema 5.2 para definir $\Delta_{q}$. Se $x \notin \bar{\Omega}_{p}$, tome $W \in \mathcal{B}_{\alpha}$ uma vizinhança aberta de $x$ contida em $V$ e disjunta de $\bar{\Omega}_{p}$. Como $V \cap K$ é aberto não-vazio em um compacto sem pontos isolados, $V \cap K$ é não-enumerável. Logo, existe $y \in V$ tal que $\mu(\{y\})=0$, para todo $\mu \in M_{p}$. Seja $\gamma=\min \left\{\varepsilon_{p}-\mu\left(\Omega_{p}\right)\right.$ : $\left.\mu \in M_{p}\right\}$. Tome $U \in \mathcal{B}_{\alpha}$ vizinhança de $y$ contida em $W$ tal que $\mu(U)<\gamma$, para todo $\mu \in M_{p}$. Tome $U^{\prime} \in \mathcal{B}_{\alpha}$ tal que $x \in U^{\prime} \subseteq \overline{U^{\prime}} \subseteq U$. Defina $q=\left(f_{p}, \Omega_{p} \cup U^{\prime}, M_{p}, \varepsilon_{p}, \Delta_{q}\right)$, obtendo $\Delta_{q}$ pelo Lema 5.2 .

Provaremos item (b). Em $M[G]$, sejam $x \in \Omega_{G} \cap \bar{K}$ e $\varepsilon>0$. Tome $p \in G$ tal que $x \in \Omega_{p}$ e $\left|\tilde{f}_{p_{1}}(y)-\tilde{f}_{p_{2}}(y)\right|<\frac{\varepsilon}{3}$, para todos $y \in \Omega_{p} \cap \bar{K}$ e $p_{1}, p_{2} \leq p$ (usamos o Lema 5.3). Usando a continuidade de $\tilde{f}_{p}$, tome $V \subseteq \Omega_{p}$ uma vizinhança aberta de $x$ tal que $\left|\tilde{f}_{p}(y)-\tilde{f}_{p}(x)\right|<\frac{\varepsilon}{3}$, para todo $y \in V \cap \bar{K}$. Teremos, para todo $q \leq p,\left|\tilde{f}_{q}(y)-\tilde{f}_{q}(x)\right| \leq\left|\tilde{f}_{q}(y)-\tilde{f}_{p}(y)\right|+\left|\tilde{f}_{p}(y)-\tilde{f}_{p}(x)\right|+$ $\left|\tilde{f}_{p}(x)-\tilde{f}_{q}(x)\right|<\varepsilon$, concluindo que $\left|f_{G}(y)-f_{G}(x)\right| \leq \varepsilon$ (pois podemos tomar $q \leq p$, tal que $\left|f_{G}(y)-f_{q}(y)\right|$ e $\left|f_{G}(x)-f_{q}(x)\right|$ são suficientemente pequenos), provando a continuidade de $f_{G}$ em $x$.

Para provarmos item (c) primeiro veremos que, se $K$ é conexo em $M$ então $\bar{K}$ é conexo em $M[G]$. De fato, se $\bar{K}$ não é conexo, pela compacidade de $\bar{K}$ existem $U$ e $V$ abertos de $[0,1]^{\alpha}$ tais que $\bar{K} \cap U \cap V=\emptyset, \bar{K} \subseteq U \cup V$, $\bar{K} \cap U \neq \emptyset$ е $\bar{K} \cap V \neq \emptyset$. Logo $K \cap U \cap V=\emptyset, K \subseteq U \cup V, K \cap U \neq \emptyset$ e $K \cap V \neq \emptyset$. Pela compacidade de $\bar{K}$ podemos assumir que $U, V \in \mathcal{B}_{\alpha}$. Portanto, como elementos de $\mathcal{B}_{\alpha}$ são determinados por coordenadas racionais, $K \cap U^{M} \cap V^{M}=\emptyset, K \subseteq U^{M} \cup V^{M}, K \cap U^{M} \neq \emptyset$ e $K \cap V^{M} \neq \emptyset$, contradizendo a conexidade de $K$ em $M$.

Mostrada a conexidade de $\bar{K}$ mostraremos que para todos $x \in \bar{K} \backslash \Omega_{G}, V$ vizinhança aberta básica de $x, r \in[0,1] \cap \mathbb{Q}$ e $n \in \omega$, existe $y \in V \cap K \cap \Omega_{G}$ tal que $\left|f_{G}(y)-r\right|<\frac{1}{n}$. Isso implicará que $\pi_{K_{G}, K}^{-1}(x)=\{x\} \times[0,1]$. Podemos assumir que $x \in K$, tomando $x^{\prime} \in V \cap K \backslash \Omega_{G}$, no lugar de $x$. Notemos que podemos assumir que $V \cap K \backslash \Omega_{G} \neq \emptyset$, pois, se $V \cap K \subseteq \Omega_{G}$, podemos assumir que $V^{M} \subseteq \Omega_{p}$, para algum $p \in G$, de onde teríamos $x \in \Omega_{G}$.

Trabalhando em $M$, dados $x \in K, V \in \mathcal{B}_{\alpha}$ vizinhança de $x, r \in[0,1] \cap \mathbb{Q} \mathrm{e}$ $p \in R(K)$, mostraremos que existe $q \leq p$ tal que $\operatorname{diam}\left(\Delta_{q}\right) \leq \frac{1}{n}$ e, ou $x \in \Omega_{q}$, ou existe $y \in V \cap \Omega_{q}$ tal que $\left|f_{q}(y)-r\right|<\frac{1}{n}$. Se $x \in \bar{\Omega}_{p}$, usando regularidade das medidas encontramos um aberto $W$ tal que $\bar{\Omega}_{p} \subseteq W$ e $\mu(W)<\varepsilon_{p}$, para todo $\mu \in M_{p}$. Tome $\Omega_{q} \in \mathcal{B}_{\alpha}$ tal que $\bar{\Omega}_{p} \subseteq \Omega_{q} \subseteq \bar{\Omega}_{q} \subseteq W, M_{q}=M_{p}, \varepsilon_{q}=\varepsilon_{p}$ e $f_{q}=f_{p}$ e usamos Lemas 5.2 e 5.1 para obtermos $\Delta_{q}$ tal que $q \in R(K)$ e 
$\operatorname{diam}\left(\Delta_{q}\right)<\frac{1}{n}$. Se $x \notin \bar{\Omega}_{p}$, tomamos $W \subseteq V$ vizinhança aberta de $x$ disjunta de $\bar{\Omega}_{p}$. Seja $y \in W$ tal que $\mu(\{y\})=0$, para todo $\mu \in M_{p}$. Seja $U$ uma vizinhança aberta de $y$ contida em $W$ tal que $\mu(U)<\varepsilon_{p}-\mu\left(\Omega_{p}\right)$, para todo $\mu \in M_{p}$. Defina $\Omega_{q}=\Omega_{p} \cup U$ e, usando o Lema de Urysohnn, construa $f_{q}: \bar{K} \longrightarrow[0,1]$ com suporte contido em $\Omega_{q}$ tal que $\left.f_{q}\right|_{\Omega_{p}}=f_{p}$ e $f_{q}(y)=r$. Defina $\varepsilon_{q}=\varepsilon_{p}$ e $M_{q}=M_{p}$ e usamos Lemas 5.1 e 5.2 para obtermos $\Delta_{q}$ tal que $\operatorname{diam}\left(\Delta_{q}\right) \leq \frac{1}{n}$.

Com isso concluímos que, em $M[G]$, se $x \in \bar{K} \backslash \Omega_{G}$, então $\pi_{K_{G}, K}^{-1}(x)=$ $\{x\} \times[0,1]$. Pelos itens (a) e (b), para $x \in \Omega_{G}$ temos $\pi_{K_{G}, K}^{-1}(x)=\left\{\left(x, f_{G}(x)\right)\right\}$. Logo $\pi_{K_{G}, K}^{-1}(x)$ é conexo, para todo $x \in K$. Suponha que $K_{G}$ não seja conexo. Sejam $F_{1}$ e $F_{2}$ fechados disjuntos não-vazios em $K_{G}$ tais que $F_{1} \cup F_{2}=K_{G}$. Pela conexidade de $K, \pi\left[F_{1}\right] \cap \pi\left[F_{2}\right] \neq \emptyset$, em $K$. Tome $x \in \pi\left[F_{1}\right] \cap \pi\left[F_{2}\right]$. Temos que $\pi^{-1}(x)$ é um compacto conexo em $K_{G}$, mas $\pi^{-1}(x) \cap F_{1}$ e $\pi^{-1}(x) \cap$ $F_{2}$ são fechados não-vazios disjuntos em $\pi^{-1}(x)$ cuja união é todo $\pi^{-1}(x)$, contradizendo a conexidade de $\pi^{-1}(x)$.

Do item a) do Lema 5.4 conluímos que $\pi_{\alpha}\left[K_{G}\right]=\bar{K}$.

Lema 5.5. Para qualquer $K \subseteq[0,1]^{\alpha}$ compacto, para $\alpha<\omega_{1}$, o forcing $R(K)$ é c.c.c.

Demonstração: Seja $\left(p_{\xi}: \xi<\omega_{1}\right)$ uma família não-enumerável de condições de $R(K)$. Podemos assumir que $\Omega_{p_{\xi}}, \varepsilon_{p_{\xi}}$ e $\Delta_{p_{\xi}}$ são constantes, em relação a $\xi$, e serão denotados, respectivamente, por $\Omega$, $\varepsilon$ e $\Delta$. Dados $\xi, \eta<\omega_{1}$ defina $p=\left(f_{p_{\xi}}, \Omega, M_{p_{\xi}} \cup M_{p_{\eta}}, \varepsilon, \Delta\right)$. É fácil verificar que $p \in R(K)$ e $p \leq p_{\xi}, p_{\eta}$.

Lema 5.6. Sejam $\varepsilon>0, p \in R(K)$ tal que $\varepsilon_{p} \leq \varepsilon, \mu \in M_{p}$ e $K \subseteq$ $[0,1]^{\alpha}$, para $\alpha<\omega_{1}$. Sejam $\left(\mu_{n}\right)_{n \in \omega}$ uma seqüência em $M\left([0,1]^{\alpha}\right)$ e $\left(x_{n}\right)_{n \in \omega}$ uma seqüência de pontos de $K$ tais que $\mu_{n}\left(\left\{x_{n}\right\}\right) \stackrel{n}{\longrightarrow} 0$, $\left(\left|\mu_{n}\right|\right)_{n \in \omega}$ converge fracamente* para $\mu,\left\{\mu_{n}: n \in \omega\right\}$ não é $5 \varepsilon$-fracamente relativamente compacto, e existem abertos $A_{n} \in \mathcal{B}_{\alpha}$ dois a dois disjuntos tais que $\left\|\mu_{n}\right\|-$ $\left|\mu_{n}\right|\left(A_{n}\right)<\frac{\varepsilon}{18} \| \mu_{n}||$.

Então existem $\delta_{1}>\delta_{2}>0$ tais que, para todo $k \in \omega$ existem $q \leq p e$ $n_{1}, n_{2}>k$ tais que

1. $\left|\int_{K} f_{q} d \mu_{n_{1}}\right|>\delta_{1}$;

2. $\left|\int_{K} f_{q} d \mu_{n_{2}}\right|<\delta_{2}$; 
3. $\left|\mu_{n_{1}}\right|\left(K \backslash \Omega_{q}\right)<\frac{\delta_{1}-\delta_{2}}{3} ;$

4. $\left|\mu_{n_{2}}\right|\left(K \backslash \Omega_{q}\right)<\frac{\delta_{1}-\delta_{2}}{3} ;$

5. $x_{n_{1}}, x_{n_{2}} \in \Omega_{q}, \mid f_{q}\left(x_{n_{1}}\right)-f_{q}\left(x_{n_{2}} \mid<\frac{1}{k}\right.$;

6. $\operatorname{diam}\left(\Delta_{q}\right) \leq \frac{1}{k}$

Demonstração: Pela Definição 4.1, passando a uma subseqüência podemos assumir que existe uma seqüência de abertos disjuntos $\left(W_{n}\right)_{n \in \omega}$ tal que $\left|\mu_{n}\left(W_{n}\right)\right|>5 \varepsilon$, para todo $n \in \omega$.

Como $\left(\mu_{n}\right)_{n \in \omega}$ não é fracamente convergente (pelo Lema 4.2), $\left\|\mu_{n}\right\|$ não converge a 0 . Passando a uma subseqüência assumimos que $\left\|\mu_{n}\right\|$ converge a $r>0$.

Tomamos $\delta_{1}=\frac{5 \varepsilon}{3} r$ e $\delta_{2}=\frac{3 \varepsilon}{2} r$. Para simplificar a notação, assumiremos que $r=1$, substituindo $\mu_{n}$ por $\frac{\mu_{n}}{r}$, para cada $n$, e $\mu$ por $\frac{\mu}{r}$. Passando a uma subseqüência e usando a hipótese, assumimos que $\left|\mu_{n}\right|\left(A_{n}\right)>1-\frac{\varepsilon}{18}$, para todo $n$.

Fixe um $\delta>0$ tal que $\delta<\frac{\varepsilon_{p}-\nu\left(\Omega_{p}\right)}{6}$, para todo $\nu \in M_{p}$. Existe tal $\delta$ pela definição de $R(K)$. Usando Lema de Rosenthal e a hipótese de que $\mu_{n}\left(\left\{x_{n}\right\}\right)$ converge a 0 , passando a uma subseqüência, assumimos que $\left|\mu_{n}\left(\left\{x_{m}\right\}\right)\right|<\delta$, para todos $n, m \in \omega$.

Fixemos $k \in \omega$. Como $\left|\mu_{n}\right|$ converge fracamente* para $\mu$, e $\mu\left(\Omega_{p}\right)<\varepsilon$ tomamos $k_{0} \geq k$ tal que, para todo $n \geq k_{0}$,

$$
\left|\mu_{n}\right|\left(\Omega_{p}\right)<\varepsilon
$$

e, portanto,

$$
\left|\int f_{p} d \mu_{n}\right|<\varepsilon
$$

Tomando $U_{n}=W_{n} \backslash \Omega_{p}$ temos, para todo $n>k_{0}$,

$$
\left|\mu_{n}\left(U_{n}\right)\right|>5 \varepsilon-\varepsilon=4 \varepsilon .
$$

Mas

$$
\left|\mu_{n}\right|\left(K \backslash A_{n}\right)<\frac{\varepsilon}{18}
$$

de onde segue que $\left|\mu_{n}\left(U_{n} \cap A_{n}\right)\right|>3 \varepsilon$. Defina $B_{n}=U_{n} \cap A_{n}$.

Como $A_{n}$ 's são dois a dois disjuntos, podemos tomar $k_{1} \geq k_{0}$ tal que, para todo $n>k_{1}, \mu\left(A_{n}\right)<\delta$ e $\nu\left(A_{n}\right)<\delta$, para todo $\nu \in M_{p}$. Assim, observando 
que $\nu\left(\Omega_{p}\right) \leq \varepsilon_{p}-4 \delta$, para todo $\nu \in M_{p}$, teremos $\nu\left(\Omega_{p} \cup A_{n} \cup A_{j}\right)<\varepsilon_{p}-2 \delta$, para todos $n, j>k_{1}$ e $\nu \in M_{p}$.

Iremos cuidar do item 5. Passando a uma subseqüência, assumimos que $x_{n}$ converge a um $x \in K$. Se $x \in \bar{\Omega}_{p}$ podemos assumir que $x \in \Omega_{p}$, estendendo $p$ para $p^{\prime}$ tal que $\bar{\Omega}_{p} \subseteq \Omega_{p^{\prime}}$ (isso pode ser feito usando regularidade das medidas). Nesse caso, passando a uma subseqüência, assumimos que $x_{n} \in \Omega_{p}$, para todo $n$, e defina $C_{n}=\emptyset$. Se $x \notin \bar{\Omega}_{p}$, assumimos que, para todo $n$, $x_{n} \notin \bar{\Omega}_{p}$ e $\left|\nu\left(\left\{x_{n}\right\}\right)\right|<\delta$, para todo $\nu \in M_{p}$. Tome $C_{n} \in \mathcal{B}_{\alpha}$ disjunto de $\bar{\Omega}_{p}$ tal que $x_{n} \in C_{n}, \nu\left(C_{n}\right)<\delta$, para todo $\nu \in M_{p}$, e $\left|\mu_{n}\right|\left(C_{m}\right)<\delta$, para todos $n, m \in \omega$. É possível escolher tais $C_{n}$ 's, pois assumimos que $\mu_{n}\left(\left\{x_{m}\right\}\right)<\delta$, para todos $n, m$.

Usando a continuidade de $f_{p}$ achamos $n_{1}, n_{2}>k_{1}$ dois inteiros distintos tais que $\left|f_{p}\left(x_{n_{1}}\right)-f_{p}\left(x_{n_{2}}\right)\right|<\frac{1}{k}$. Defina

$$
\Omega_{q}=\Omega_{p} \cup A_{n_{1}} \cup A_{n_{2}} \cup C_{n_{1}} \cup C_{n_{2}} .
$$

Como $\left|\mu_{n}\left(B_{n}\right)\right|>3 \varepsilon$, pela regularidade de $\mu_{n}$ achamos um fechado $F_{n} \subseteq$ $B_{n}$ tal que $\left|\mu_{n}\left(F_{n}\right)\right|>3 \varepsilon$.

Tome $f: K \longrightarrow[0,1]$ contínua tal que $\left.f\right|_{F_{n_{1}}}=1$ e $\left.f\right|_{K \backslash B_{n_{1}}}=\left.f_{p}\right|_{K \backslash B_{n_{1}}}$ e $g: K \longrightarrow[0,1]$ contínua tal que $\left.g\right|_{K \backslash\left(C_{n_{1}} \cup C_{n_{2}}\right)}=1$ e $g\left(x_{n_{1}}\right)=g\left(x_{n_{2}}\right)=0$, no caso $C_{n} \neq \emptyset$, e $g=1$ caso contrário. Defina $f_{q}=f \cdot g, M_{q}=M_{p}, \varepsilon_{q}=\varepsilon_{p}$ e construa $\Delta_{q}$ como no lema 5.2. Pelo Lema 5.1 assumimos que $\operatorname{diam}\left(\Delta_{q}\right)<\frac{1}{k}$. É fácil ver que $q \in R(K)$ e $\left.f_{q}\right|_{\Omega_{p}}=\left.f_{p}\right|_{\Omega_{p}}$, pois $B_{n} \cap \Omega_{p}=\emptyset$ Logo $q \leq p$. Temos $\left|\int f_{q} d \mu_{n_{1}}\right| \geq\left|\mu_{n_{1}}\left(F_{n_{1}}\right)\right|-\left|\mu_{n_{1}}\right|\left(\Omega_{p}\right)-\left|\mu_{n_{1}}\right|\left(C_{n_{1}} \cup C_{n_{2}}\right)>3 \varepsilon-\varepsilon-2 \delta \geq \frac{5 \varepsilon}{3}$

$\mathrm{e}$

$$
\begin{gathered}
\left|\int f_{q} d \mu_{n_{2}}\right| \leq\left|\mu_{n_{2}}\left(B_{n_{1}}\right)\right|+\left|\int f_{p} d \mu_{n_{2}}\right|+\left|\mu_{n_{2}}\right|\left(C_{n_{1}} \cup C_{n_{2}}\right) \\
<\frac{\varepsilon}{18}+\varepsilon+2 \delta<\frac{3 \varepsilon}{2} .
\end{gathered}
$$

Como $A_{n_{1}} \cup A_{n_{2}} \subseteq \Omega_{q}$, temos

$$
\left|\mu_{n}\right|\left(K \backslash \Omega_{q}\right)<\frac{\varepsilon}{18}=\frac{\delta_{1}-\delta_{2}}{3},
$$

para $n \in\left\{n_{1}, n_{2}\right\}$. Concluímos, portanto, os itens 1 a 4 . O item 5 segue da escolha de $k_{1}$ e $k_{2}$, no caso $x \in \Omega_{p}$, pois teremos $f_{q}\left(x_{n}\right)=f_{p}\left(x_{n}\right)$. No caso $x \notin \Omega_{p}$, isto é, $C_{n} \neq \emptyset$, o item 5 segue de que $f_{q}\left(x_{n_{1}}\right)=f_{q}\left(x_{n_{2}}\right)=0$. O item 6 é segue da construção de $\Delta_{q}$. 


\subsection{Iteração do forcing}

Construiremos indutivamente forcings $\left(P_{\alpha}\right)_{\alpha \leq \omega_{1}}$ juntamente com $P_{\alpha}$-nomes $\left(\dot{K}_{\alpha}\right)_{\alpha \leq \omega_{1}}$ tais que $P_{\alpha} \Vdash$ " $\dot{K}_{\alpha}$ é compacto de peso enumerável". Tome $P_{0}$ um forcing trivial e $K_{0}=[0,1]^{2}$. Definidos $P_{\alpha}$ e $\dot{K}_{\alpha}$, definimos

$$
P_{\alpha+1}=P_{\alpha} * \dot{Q}_{\alpha}
$$

onde $\dot{Q}_{\alpha}$ é um $P_{\alpha}$-nome tal que

$$
P_{\alpha} \Vdash \dot{Q}_{\alpha}=R\left(\dot{K}_{\alpha}\right)
$$

e definimos $\dot{K}_{\alpha+1}$ um $P_{\alpha+1}$-nome tal que $P_{\alpha+1} \Vdash \dot{K}_{\alpha+1}=\left(\dot{K}_{\alpha}\right)_{\dot{G}_{\alpha}}$. Se $\alpha \leq \omega_{1}$ é um ordinal limite e $\left(P_{\beta}\right)_{\beta<\alpha}$ e $\left(\dot{K}_{\beta}\right)_{\beta<\alpha}$ estão definidos, definimos $P_{\alpha}$ a iteração com suporte finito de $\left(P_{\beta}\right)_{\beta<\alpha}$ e $\dot{K}_{\alpha}$ tal que

$$
P_{\alpha} \Vdash \dot{K}_{\alpha}=\lim _{\leftarrow}\left(\overline{\dot{K}}_{\beta}\right)_{\beta<\alpha}
$$

Seja $P=P_{\omega_{1}}$. Em $M\left[G_{\alpha}\right]$ considere $K_{\alpha}=\left(\dot{K}_{\alpha}\right)_{G_{\alpha}}$.

Pela definição temos $K_{\alpha} \subseteq[0,1]^{\alpha}$, para $\alpha \geq \omega$, e $K_{\alpha} \subseteq[0,1]^{\alpha+2}$, se $\alpha<\omega$. Para termos uma notação uniforme, se $\alpha<\omega$ e $x \in K_{\gamma}$, para $\gamma>\alpha$, denotamos $\left.x\right|_{\alpha+2}$ por $\left.x\right|_{\alpha}$.

Como $P$ é uma iteração com suportes finitos de forcings c.c.c. $P$ também é c.c.c. e portanto, preserva cardinais.

Em $K_{\alpha}$ definimos $\left(q_{n} \mid \alpha\right)_{n \in \omega} \subseteq K_{\alpha}$ indutivamente, em $M_{\alpha}$. Em $M$, fixa$\operatorname{mos}\left\{q_{n}(0): n \in \omega\right\}$ uma enumeração dos pares de racionais em $[0,1]^{2}$. Definido $\left\{q_{n}(\alpha): n \in \omega\right\}$ em $M_{\alpha}$, em $M_{\alpha+1}$ definimos $q_{n} \mid(\alpha+1)=\left(q_{n}(\alpha), f_{G}\left(q_{n} \mid \alpha\right)\right)$, se $q_{n} \mid \alpha \in \Omega_{G_{\{\alpha\}}}$, e $q_{n} \mid(\alpha+1)=\left(q_{n} \mid \alpha, 0\right)$, caso contrário. Para $\alpha$ limite defini$\operatorname{mos} q_{n} \mid \alpha=\bigcup_{\beta<\alpha} q_{n}(\beta)$. Seja $\dot{q}_{n}(\alpha)$ um $P_{\alpha}$-nome para $q_{n} \mid \alpha$. Seja $q_{n}=q_{n}\left(\omega_{1}\right)$ em $M_{\omega_{1}}$.

Lema 5.7. Em $M[G]$, o conjunto $\left\{q_{n}: n \in \omega\right\}$ é denso em $K_{\omega_{1}}$.

Demonstração: Por hipótese $\left\{q_{n} \mid 0: n \in \omega\right\}$ é denso em $K_{0}$. Se $\left\{q_{n} \mid \beta\right.$ : $n \in \omega\}$ é denso em $K_{\beta}$, para todo $\beta<\alpha$ e $\alpha$ um ordinal limite, então, em $M_{\alpha}$, $\left\{q_{n} \mid \alpha: n \in \omega\right\}$ é denso em $K_{\alpha}$. De fato, se existe $V \in \mathcal{B}_{\alpha}$ não-vazio tal que $V$ é disjunto de $\left\{q_{n} \mid \alpha: n \in \omega\right\}$, como $V$ é união finita de abertos elementares, existe $\beta<\alpha$ tal que $\pi_{\beta}[V]$ é aberto não-vazio em $K_{\beta}$, contradizendo que $\left\{q_{n} \mid \beta: n \in \omega\right\}$ é denso em $K_{\beta}$. 
Suponha que $\left\{q_{n} \mid \alpha: n \in \omega\right\}$ é denso em $K_{\alpha}$. Seja $V$ aberto não-vazio de $K_{\alpha+1}$. Como $G r\left(f_{G_{\{\alpha\}}}\right)$ é denso em $K_{\alpha+1}$ então $V$ intercepta $G r\left(f_{G_{\{\alpha\}}}\right)=$ $\pi^{-1}\left(\Omega_{G_{\{\alpha\}}}\right)$, que é aberto em $K_{\alpha+1}$. Logo, tomando essa intersecção no lugar de $V$, assumimos que $V \subseteq G r\left(f_{G_{\{\alpha\}}}\right)$. Como, pelo Lema 5.4, $f_{G_{\{\alpha\}}}$ é contínua em $\Omega_{G_{\{\alpha\}}}$, temos que $\pi_{K_{\alpha+1}, K_{\alpha}}^{-1}$ é um homeomorfismo, quando restrito a $\Omega_{G_{\{\alpha\}}}$, de onde segue que $\pi_{K_{\alpha+1}, K_{\alpha}}[V]$ é aberto em $K_{\alpha}$ e, portanto, irá interceptar $\left\{q_{n} \mid \alpha: n \in \omega\right\}$, concluindo que $V$ intercepta $\left\{q_{n} \mid(\alpha+1): n \in \omega\right\}$.

Lema 5.8. Em $M[G]$, o espaço $K_{\omega_{1}}$ é conexo.

Demonstração: Procedemos por indução. Para $\alpha=0$ temos $K_{\alpha}$ conexo, em $M_{0}$, pois $K_{0}=[0,1]^{2}$. Se $K_{\alpha}$ é conexo em $M_{\alpha}$, pelo Lema 5.4 temos que $K_{\alpha+1}$ é conexo em $M_{\alpha+1}$. Seja $\alpha \leq \omega_{1}$ um ordinal limite e suponha que, em $M_{\beta}, K_{\beta}$ é conexo, para todo $\beta<\alpha$. Suponha que $K_{\alpha}$ não seja conexo. Pela compacidade de $K_{\alpha}$ existem $U, V \in \mathcal{B}_{\alpha}$ tais que $K_{\alpha} \cap U \cap V=\emptyset, K_{\alpha} \subseteq U \cup V$, $K_{\alpha} \cap U \neq \emptyset$ e $K_{\alpha} \cap V \neq \emptyset$. Como elementos de $\mathcal{B}_{\alpha}$ são determinados por finitas coordenadas de $\alpha$, existe $\beta<\alpha$ tal que $\pi_{\beta}[U]$ e $\pi_{\beta}[V]$ são abertos tais que $U=\pi_{\beta}^{-1}\left[\pi_{\beta}[U]\right]$ e $V=\pi_{\beta}^{-1}\left[\pi_{\beta}[V]\right]$, o que daria que $\bar{K}_{\beta}$, e, portanto, $K_{\beta}$, não é conexo.

Lema 5.9. Sejam $\dot{\mu}$ um P-nome para uma medida em $K_{\omega_{1}}$ e seja $\dot{z}$ um $P$-nome para um ponto de $K_{\omega_{1}}$. Então os seguintes conjuntos são fechados ilimitados em $\omega_{1}$.

a) $C_{\dot{\mu}}=\left\{\alpha<\omega_{1}:\left.P \Vdash \dot{\mu}\right|_{\mathcal{B}_{\alpha}} \in M_{\alpha} e|\dot{\mu}|_{\mid \mathcal{B}_{\alpha}}=|\dot{\mu}|_{\mathcal{B}_{\alpha}} \mid\right\}$;

b) $C_{\dot{z}}=\left\{\alpha<\omega_{1}:\left.P \Vdash \dot{z}\right|_{\alpha} \in M_{\alpha}\right\}$

Demonstração: Análoga à do Lema 4.9

Se $G$ é um $R(K)$-genérico sobre $M$, em $M[G]$ definimos $f_{G}^{\prime}: K_{G} \longrightarrow[0,1]$ por $f_{G}^{\prime}(x, t)=t$, para $(x, t) \in K_{G} \subseteq \bar{K} \times[0,1]$. Em $M$ tomamos $\dot{f}_{G}^{\prime}$ um $R(K)$-nome para $f_{G}^{\prime}$. Observamos que

$$
\left.f_{G}^{\prime}\right|_{K_{G} \cap \Omega_{G} \times[0,1]}=f_{G} \circ \pi,
$$

uma vez que $\pi_{K_{G}, \bar{K}}^{-1}(x)=\left\{f_{G}(x)\right\}$, para $x \in \Omega_{G}$.

Seja $G$ um $P$-genérico sobre $M$ e seja $\alpha<\omega_{1}$. Em $M[G]$ definimos $\tilde{f}_{G_{\{\alpha\}}}^{\prime}$ a extensão contínua de $f_{G_{\{\alpha\}}}^{\prime}$ em $\bar{K}_{\alpha}^{M[G]}$, que existe porque $f_{G_{\{\alpha\}}}^{\prime}$ é uniformemente contínua em $K_{\alpha}$. Seja $\bar{f}_{G_{\{\alpha\}}}^{\prime}$ um $P$-nome para $\tilde{f}_{G_{\{\alpha\}}}^{\prime}$. 
Lema 5.10. Sejam $\varepsilon>0$ racional, $\alpha<\omega_{1}$ e $p \in P$ tais que $\varepsilon_{p(\alpha)} \leq \varepsilon$ e $\dot{\mu} \in M_{p}$, para $\dot{\mu}$ um $P_{\alpha}$-nome. Sejam $\left(\dot{\mu}_{n}\right)_{n \in \omega}$ e $\left(\dot{x}_{n}\right)_{n \in \omega}$ seqüências de $P$-nomes tais que $p$ força:

1. $\dot{\mu}_{n} \in M\left(K_{\omega_{1}}\right)$;

2. $\dot{x}_{n} \in K_{\omega_{1}}$;

3. $\left.\mu_{n}\right|_{\mathcal{B}_{\alpha}} \in M_{\alpha}$;

4. $\left.\dot{x}_{n}\right|_{\alpha} \in M_{\alpha}$;

5. $\left|\dot{\mu}_{n \mid \mathcal{B}_{\alpha}}\right|=\left|\dot{\mu}_{n}\right| \mathcal{B}_{\alpha} ;$

6. $\left.\dot{\mu}_{n}\right|_{\mathcal{B}_{\alpha}}\left(\left\{\left.\dot{x}_{n}\right|_{\alpha}\right\}\right) \stackrel{n}{\longrightarrow} 0$;

7. $\left|\dot{\mu}_{n}\right|_{\mathcal{B}_{\alpha}} \mid$ converge fracamente ${ }^{*}$ para $\dot{\mu}$;

8. $\left\{\left.\dot{\mu}_{n}\right|_{\mathcal{B}_{\alpha}}: n \in \check{\omega}\right\}$ ñ̃o é $\check{5} \check{\varepsilon}$-fracamente relativamente compacto;

9. existe uma seqüência $\left(A_{n}\right)_{n \in \omega} \subseteq \mathcal{B}_{\alpha}$ dois a dois disjuntos tal que $\left\|\dot{\mu}_{n}\right\|-$ $\left|\dot{\mu}_{n}\right|\left(A_{n}\right)<\frac{\varepsilon}{18} \| \dot{\mu}_{n}||$, para todo $n$.

Então existem $\delta_{1}, \delta_{2}>0$ tais que $p \Vdash \forall k \in \check{\omega} \exists n_{1}, n_{2}>k$

$\left|\int_{K} \bar{f}_{G_{\{\alpha\}}}^{\prime} \circ \pi_{\alpha+1} d \dot{\mu}_{n_{1}}\right|>\check{\delta}_{1},\left|\int_{K} \bar{f}_{G_{\{\alpha\}}}^{\prime} \circ \pi_{\alpha+1} d \dot{\mu}_{n_{2}}\right|<\check{\delta}_{2} e \mid \dot{f}_{G_{\{\alpha\}}}\left(\left.\dot{x}_{n_{1}}\right|_{\alpha}\right)-$ $\dot{f}_{G_{\{\alpha\}}}\left(\dot{x}_{n_{2}} \mid \alpha\right) \mid<\frac{2}{k}$.

Demonstração: Seja $G$ um $P$-genérico sobre um modelo inicial $M$ tal que $p \in G$. Em $M[G]$, sejam $\mu_{n}=\left(\dot{\mu}_{n}\right)_{G}$ e $x_{n}=\left(\dot{x}_{n}\right)_{G}$. Em $M\left[G_{\alpha}\right]$ tome $\mu=\dot{\mu}_{G_{\{\alpha\}}}$.

Sabendo, pela hipótese, que $\mu_{n \mid \mathcal{B}_{\alpha}} \in M\left[G_{\alpha}\right]$ e $\left.x_{n}\right|_{\alpha} \in M\left[G_{\alpha}\right]$, para todo $n$, trabalhemos em $M\left[G_{\alpha}\right]$. Pelo Lema 5.6, existem $\delta_{1}^{\prime}>\delta_{2}^{\prime}>0$ tais que para todos $p^{\prime} \leq p(\alpha) \in R\left(K_{\alpha}\right)$ e $k \in \omega$, existem $n_{1}, n_{2}>k$ e $q \leq p^{\prime}$ tais que $\left|\int f_{q} d \mu_{n_{1} \mid \mathcal{B}_{\alpha}}\right|>\delta_{1}^{\prime},\left|\int f_{q} d \mu_{n_{2} \mid \mathcal{B}_{\alpha}}\right|<\delta_{2}^{\prime},\left|\mu_{n_{i} \mid \mathcal{B}_{\alpha}}\right|\left(K_{\alpha} \backslash \Omega_{q}\right)<\frac{\delta_{1}^{\prime}-\delta_{2}^{\prime}}{3}$, para $i=1,2$. Pelo Lema 5.1 podemos assumir que

$$
\operatorname{diam}\left(\Delta_{q}\right) \leq \min \left\{\frac{\left|\int f_{q} d \mu_{n_{1} \mid \mathcal{B}_{\alpha}}\right|-\delta_{1}^{\prime}}{\| \mu_{n_{1} \mid \mathcal{B}_{\alpha}}||}, \frac{\delta_{2}^{\prime}-\left|\int f_{q} d \mu_{n_{2} \mid \mathcal{B}_{\alpha}}\right|}{|| \mu_{n_{2} \mid \mathcal{B}_{\alpha}}||}\right\}
$$

Portanto, para todo $r \leq q$, temos

$$
\left|\int_{\Omega_{q}} f_{r} d \mu_{n_{1} \mid \mathcal{B}_{\alpha}}\right|>\left|\int f_{q} d \mu_{n_{1}}\right|-\operatorname{diam}\left(\Delta_{q}\right) \cdot\left|\mu_{n_{1} \mid \mathcal{B}_{\alpha}}\right|\left(\Omega_{q}\right) \geq \delta_{1}^{\prime}
$$


e

$$
\left|\int_{\Omega_{q}} f_{r} d \mu_{n_{2}}\right|<\left|\int f_{q} d \mu_{n_{2} \mid \mathcal{B}_{\alpha}}\right|+\operatorname{diam}\left(\Delta_{q}\right) \cdot\left|\mu_{n_{2} \mid \mathcal{B}_{\alpha}}\right|\left(\Omega_{q}\right) \leq \delta_{2}^{\prime} .
$$

Observamos que, como $\left.\mu_{n}\right|_{\mathcal{B}_{\alpha}} \in M\left[G_{\alpha}\right]$, em $M[G]$ temos

$$
\left.\int_{\Omega^{M[G] \cap \bar{K}}} \tilde{f}_{r} d \mu_{n}\right|_{\mathcal{B}_{\alpha}}=\left.\int_{\Omega^{M} \cap K} f_{r} d \mu_{n}\right|_{\mathcal{B}_{\alpha}},
$$

para todo $\Omega \in \mathcal{B}_{\alpha}$ e portanto, em $M[G]$,

$$
\begin{aligned}
& \left|\int_{\Omega_{r} \cap \bar{K}_{\alpha}} \tilde{f}_{r} d \mu_{n_{1} \mid \mathcal{B}_{\alpha}}\right|>\delta_{1}^{\prime} \\
& \left|\int_{\Omega_{r} \cap \bar{K}_{\alpha}} \tilde{f}_{r} d \mu_{n_{2} \mid \mathcal{B}_{\alpha}}\right|<\delta_{2}^{\prime}
\end{aligned}
$$

e

$$
\left|\mu_{n_{i} \mid \mathcal{B}_{\alpha}}\right|\left(\bar{K}_{\alpha} \backslash \Omega_{r}\right)<\frac{\delta_{1}^{\prime}-\delta_{2}^{\prime}}{3}
$$

para $i \in\{1,2\}$. Notemos que, aqui, $\tilde{f}_{r}$ representa a extensão contínua de $f_{r}$ em $K_{\alpha}$ em $\bar{K}_{\alpha}^{M[G]}$, e não em $\bar{K}_{\alpha}^{M\left[G_{\alpha+1}\right]}$, conforme a definição anterior. Da mesma forma, interpretamos $f_{G_{\{\alpha\}}}$ como uma função contínua de $\Omega_{G_{\{\alpha\}}} \cap$ $\bar{K}_{\alpha+1}^{M[G]}$ em $[0,1]$.

As desigualdades (1) e (2) valem também para $f_{G_{\{\alpha\}}}$ no lugar de $\tilde{f}_{r}$, pois, pela definição de $f_{G_{\{\alpha\}}}$, para todos $x \in \Omega_{G_{\{\alpha\}}}$ e $\varepsilon^{\prime}>0$ existe $r \leq p(\alpha)$ tal que para todo $s \leq r$ temos $\left|f_{G}(x)-f_{s}(x)\right|<\varepsilon^{\prime}$.

De (3) e da hipótese 5 concluímos que $\left|\mu_{n_{i}}\right|\left(K \backslash \Omega_{r} \times[0,1]^{\omega_{1} \backslash \alpha}\right)<\frac{\delta_{1}^{\prime}-\delta_{2}^{\prime}}{3}$, para $i \in\{1,2\}$, e de (1) e (2) para $f_{G}$ e de $(* *)$ concluímos que

$$
\left|\int_{K \cap \Omega_{r} \times[0,1]^{\omega_{1} \backslash \alpha}} f_{G_{\{\alpha\}}}^{\prime} \circ \pi_{\alpha+1} d \mu_{n_{1}}\right|>\delta_{1}^{\prime}
$$

e

$$
\left|\int_{K \cap \Omega_{r} \times[0,1]^{\omega_{1} \backslash \alpha}} f_{G_{\{\alpha\}}}^{\prime} \circ \pi_{\alpha+1} d \mu_{n_{2}}\right|<\delta_{2}^{\prime} .
$$

$\operatorname{Logo}$

$$
\left|\int_{K} f_{G_{\{\alpha\}}}^{\prime} \circ \pi_{\alpha+1} d \mu_{n_{1}}\right|>\delta_{1}^{\prime}-\frac{\delta_{1}^{\prime}-\delta_{2}^{\prime}}{3}
$$


e

$$
\left|\int_{K} f_{G_{\{\alpha\}}}^{\prime} \circ \pi_{\alpha+1} d \mu_{n_{2}}\right|<\delta_{2}^{\prime}+\frac{\delta_{1}^{\prime}-\delta_{2}^{\prime}}{3} .
$$

Assim, tomando $\delta_{1}=\delta_{1}^{\prime}-\frac{\delta_{1}^{\prime}-\delta_{2}^{\prime}}{3}$ e $\delta_{2}=\delta_{2}^{\prime}+\frac{\delta_{1}^{\prime}-\delta_{2}^{\prime}}{3}$ temos que $\delta_{1}>\delta_{2}>0$ satisfazem a tese do lema. A segunda parte do lema segue dos itens 5 e 6 do Lema 5.6.

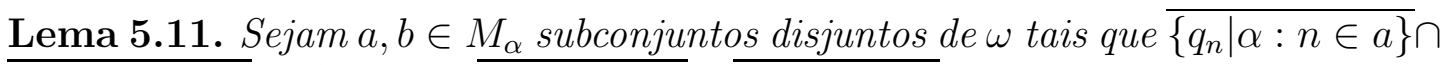
$\overline{\left\{q_{n} \mid \alpha: n \in b\right\}} \neq \emptyset$. Então $\overline{\left\{q_{n}: n \in a\right\}} \cap \overline{\left\{q_{n}: n \in b\right\}} \neq \emptyset$, em $M_{\omega_{1}}$.

Demonstração: Observamos que, se $\overline{\left\{q_{n} \mid \beta: n \in a\right\}} \cap \overline{\left\{q_{n} \mid \beta: n \in b\right\}}=\emptyset$, para $\beta$ um ordinal limite, existe $\gamma<\beta$ em que a separação acontece, no modelo $M_{\beta}$. Portanto, para mostrar o lema, é suficiente mostrar que $\overline{\left\{q_{n} \mid(\alpha+1): n \in a\right\}} \cap$ $\overline{\left\{q_{n} \mid(\alpha+1): n \in b\right\}} \neq \emptyset$, e prosseguimos por indução.

Sejam $\alpha<\omega_{1}$ e $a, b \subseteq \omega$ como na hipótese do lema. Trabalharemos em $M_{\alpha}$. Mostraremos então que

$$
R\left(K_{\alpha}\right) \Vdash \overline{\left\{\dot{q}_{n} \mid(\alpha+1): n \in \check{a}\right\}} \cap \overline{\left\{\dot{q}_{n} \mid(\alpha+1): n \in \check{b}\right\}} \neq \emptyset, \text { em } \dot{K}_{\alpha+1} .
$$

Seja $p \in R\left(K_{\alpha}\right)$. Mostraremos que existe $q \leq p$ tal que $q \Vdash \overline{\left\{\dot{q}_{n} \mid(\alpha+1): n \in \check{a}\right\}} \cap$ $\overline{\left\{\dot{q}_{n} \mid(\alpha+1): n \in \check{b}\right\}} \neq \emptyset$.

Usando que $K_{\alpha}$ é metrizável e passando a uma subseqüência, assumimos que existe $z \in K_{\alpha}$ tal que $q_{n} \mid \alpha$ converge a $z$.

Consideramos dois casos. Se existe $q \leq p$ tal que $z \in \bar{\Omega}_{q}$, usando regularidade das medidas em $M_{q}$ podemos assumir que $z \in \Omega_{q}$. Como $f_{G_{\{\alpha\}}}$ é contínua em $\Omega_{G_{\{\alpha\}}}$, e $R\left(K_{\alpha}\right) \Vdash \dot{K}_{\alpha+1}=\overline{G r\left(\dot{f}_{G_{\{\alpha\}}}\right)}$, temos que $q \Vdash$ $\overline{\left\{\dot{q}_{n} \mid(\alpha+1): n \in \check{a}\right\}} \cap \overline{\left\{\dot{q}_{n} \mid(\alpha+1): n \in \check{b}\right\}} \neq \emptyset$.

No segundo caso, para todo $q \leq p$ temos $z \notin \bar{\Omega}_{q}$. Sejam $q \leq p$ e $k \in \omega$. Tome $V$ vizinhança aberta de $z$ disjunta de $\bar{\Omega}_{q}$. Como $\mu\left(\left\{q_{n} \mid \alpha\right\}\right) \stackrel{n \in \omega}{\longrightarrow} 0$, para todo $\mu \in M_{q}$, podemos achar $n_{1}, n_{2}>k$ e abertos disjuntos $U_{1}, U_{2} \subseteq V$ tais que

1. $n_{1} \in a, n_{2} \in b$;

2. $q_{n_{1}}\left|\alpha \in U_{1}, q_{n_{2}}\right| \alpha \in U_{2}$;

3. $\mu\left(\overline{U_{1} \cup U_{2}}\right)<\varepsilon_{q}-\mu\left(\Omega_{q}\right)$, para todo $\mu \in M_{q}$. 
Tome $\Omega_{r}=\Omega_{q} \cup U_{1} \cup U_{2}, f_{r}=f_{q}, \varepsilon_{r}=\varepsilon_{q}, M_{r}=M_{q}$ e $\Delta_{r}$ tal que $r \in R\left(K_{\alpha}\right)$ e $\operatorname{diam}\left(\Delta_{r}\right) \leq \frac{1}{k}$. Pelas condições acima temos que $r \in R\left(K_{\alpha}\right)$, $r \leq q$ e, para todo $i \in\{1,2\}$,

$$
r \Vdash \check{q}_{n_{i}} \mid \alpha \in \Omega_{G_{\{\alpha\}}} \text { e } \dot{f}_{G_{\{\alpha\}}}\left(\check{q}_{n_{i}} \mid \alpha\right)<\frac{1}{k}
$$

mostrando que

$$
p \Vdash(\check{z}, 0) \in \overline{\left\{\dot{q}_{n} \mid(\alpha+1): n \in \check{a}\right\}} \cap \overline{\left\{\dot{q}_{n} \mid(\alpha+1): n \in \check{b}\right\}} .
$$

Lema 5.12. Se $U, V$ são abertos disjuntos em $K_{\omega_{1}}$ tais que $\bar{U} \cap \bar{V} \neq \emptyset$, então $\bar{U} \cap \bar{V}$ não é unitário.

Demonstração: Sejam $\dot{U}$ e $\dot{V} P$-nomes para abertos disjuntos de $K_{\omega_{1}}$ tais que

$$
P \Vdash \overline{\dot{U}} \cap \overline{\dot{V}} \neq \emptyset .
$$

Para $\alpha<\omega_{1}$, sejam $\dot{U}_{\alpha}$ e $\dot{V}_{\alpha} P$-nomes tais que

$$
P \Vdash \pi_{\alpha}[\dot{U}]=\dot{U}_{\alpha}, \pi_{\alpha}[\dot{V}]=\dot{V}_{\alpha} .
$$

Como $K_{\omega_{1}}$ é separável, se $V$ é aberto de $K_{\omega_{1}}$ existe $V^{\prime}$ união enumerável de abertos básicos tal que $\bar{V}=\overline{V^{\prime}}$. Assim, podemos assumir que, em $M[G]$, $(\dot{U})_{G}$ e $(\dot{V})_{G}$ são uniões enumeráveis de abertos básicos. Tomemos $\gamma<\omega_{1}$ e $p \in P$ tais que

$$
p \Vdash \forall \alpha \geq \gamma\left(\dot{U}=\pi_{\alpha}^{-1}\left[\dot{U}_{\alpha}\right] \text { e } \dot{V}=\pi_{\alpha}^{-1}\left[\dot{V}_{\alpha}\right]\right) .
$$

Seja $q \leq p$. Seja $\dot{z}$ um $P$-nome para um ponto de $K$ tal que

$$
q \Vdash \dot{z} \in \overline{\dot{U}} \cap \overline{\dot{V}} .
$$

Tome $\alpha>\gamma, \operatorname{supp}(q)$ tal que $\left.q \Vdash \dot{z}\right|_{\alpha} \in M_{\alpha}$, o que é possível pelo Lema 5.9.

Pelo Lema 5.7, e como $K_{\alpha}$ é metrizável, existem $P$-nomes $\dot{a}$ e $\dot{b}$ para subconjuntos disjuntos de $\omega$ tais que $q \Vdash \dot{a}, \dot{b} \in M_{\alpha} \mathrm{e}$

$$
q \Vdash\left\{\dot{q}_{n}: n \in \dot{a}\right\} \subseteq \dot{U},\left\{\dot{q}_{n}: n \in \dot{b}\right\} \subseteq \dot{V}, \dot{q}_{n}|\alpha \stackrel{n \in \dot{a}}{\longrightarrow} \dot{z}|_{\alpha}, \dot{q}_{n}|\alpha \stackrel{n \in \dot{b}}{\longrightarrow} \dot{z}|_{\alpha} .
$$


Seja $r \leq q$ definido por $r(\beta)=q(\beta)$, se $\beta \neq \alpha, f_{r(\alpha)}$ a função identicamente nula, $\Omega_{r(\alpha)}=\emptyset, M_{r(\alpha)}=\left\{\delta_{\left.\dot{z}\right|_{\alpha}}\right\}, \Delta_{r(\alpha)}=\emptyset \mathrm{e} \varepsilon_{r(\alpha)}=1$.

$\operatorname{Em} M\left[G_{\alpha}\right]$, iremos denotar $\left(\left.\dot{z}\right|_{\alpha}\right)_{G_{\alpha}}$ por $\left.z\right|_{\alpha}$.

Em $R\left(K_{\alpha}\right)$, no modelo $M_{\alpha}$, não existe $s \leq r(\alpha)$ tal que $\left.z\right|_{\alpha} \in \Omega_{s}$, pois teríamos $\delta_{\left.z\right|_{\alpha}}\left(\Omega_{s}\right)=1$. Assim, analogamente à demonstração do Lema 5.11, concluímos que, em $M_{\alpha}$,

$$
r(\alpha) \Vdash\left(\left.\dot{z}\right|_{\alpha}, 0\right) \in \overline{\left\{\check{q}_{n} \mid(\alpha+1): n \in \check{a}\right\}} \cap \overline{\left\{\check{q}_{n} \mid(\alpha+1): n \in \check{b}\right\}},
$$

onde $a=\dot{a}_{G_{\alpha}}$ e $b=\dot{b}_{G_{\alpha}}$.

Retomando o último parágrafo da demonstração do Lema 5.11, podemos modificá-lo tomando $f_{r}$ tal que $f_{r}\left(q_{i} \mid \alpha\right)=1$, para $i \in\left\{n_{1}, n_{2}\right\}$ e $\left.f_{r}\right|_{K \backslash\left(U_{1} \cup U_{2}\right)}=\left.f_{q}\right|_{K \backslash\left(U_{1} \cup U_{2}\right)}$, ao invés de $f_{r}=f_{q}$. Construindo assim $r(\alpha) \in$ $R\left(K_{\alpha}\right)$ em $M\left[G_{\alpha}\right]$ teremos

$$
r(\alpha) \Vdash \check{q}_{n_{i}} \mid \alpha \in \Omega_{G_{\{\alpha\}}} \text { e }\left|f_{G_{\{\alpha\}}}\left(\check{q}_{n_{i}} \mid \alpha\right)-1\right|<\frac{1}{k}
$$

e, portanto,

$$
r(\alpha) \Vdash\left(\left.\dot{z}\right|_{\alpha}, 1\right) \in \overline{\left\{\check{q}_{n} \mid(\alpha+1): n \in \check{a}\right\}} \cap \overline{\left\{\check{q}_{n} \mid(\alpha+1): n \in \check{b}\right\}} .
$$

Concluímos, então, que

$$
r \Vdash \pi_{\alpha+1}^{-1}\left[\left(\left.\dot{z}\right|_{\alpha}, 0\right)\right] \subseteq \overline{\dot{U}} \cap \overline{\dot{V}}, \pi_{\alpha+1}^{-1}\left[\left(\left.\dot{z}\right|_{\alpha}, 1\right)\right] \subseteq \overline{\dot{U}} \cap \overline{\dot{V}}
$$

e, portanto,

$$
r \Vdash|\overline{\dot{U}} \cap \overline{\dot{V}}| \geq 2
$$

Lema 5.13. Todo operador em $C\left(K_{\omega_{1}}\right)$ é multiplicador fraco.

Demonstração: Sejam $p \in P$ e $G$ um $P$-genérico sobre um modelo inicial $M$. Trabalhemos em $M[G]$. Seja $K=K_{\omega_{1}}$. Identificaremos $M(K)$ com o conjunto $\left\{\mu \in M\left([0,1]^{\omega_{1}}\right):|\mu|\left([0,1]^{\omega_{1}} \backslash K\right)=0\right\}$. Consideramos $\mathcal{B}=\mathcal{B}_{\omega_{1}}$.

Suponha que exista $T: C(K) \longrightarrow C(K)$ que não é multiplicador fraco. Pelos Lemas 4.3 e 5.7 existem $x_{n} \in\left\{q_{m}: m \in \omega\right\}$ distintos tais que, para toda função boreliana $f: K \longrightarrow \mathbb{R}$ limitada, $\left\{T^{*}\left(\delta_{x_{n}}\right)-f \delta_{x_{n}}: n \in \omega\right\}$ não é relativamente fracamente compacto. Tome $f: K \longrightarrow \mathbb{R}$ definida por 
$f\left(x_{n}\right)=T^{*}\left(\delta_{x_{n}}\right)\left(\left\{x_{n}\right\}\right)$, para todo $n \in \omega$, e $f(x)=0$ nos demais pontos de $K$. Passando a uma subseqüência assumimos que $f\left(x_{n}\right)$ converge a $L \in \mathbb{R}$. Pelo Corolário 4.6 existem seqüências de medidas $\left(\mu_{n}\right)_{n \in \omega}$ e $\left(\lambda_{n}\right)_{n \in \omega}$ tais que $\left(\mu_{n}\right)_{n \in \omega}$ são duas a duas disjuntas, $\left(\lambda_{n}\right)_{n \in \omega}$ converge fracamente para $\lambda \in M(K) \mathrm{e}$

$$
T^{*}\left(\delta_{x_{n}}\right)-f \delta_{x_{n}}=\mu_{n}+\lambda_{n}
$$

Sejam $\dot{\mu}_{n} P$-nomes para $\mu_{n}$ e $\dot{x}_{n} P$-nomes para $x_{n}$.

Considere $C=\bigcap_{n \in \omega} C_{\dot{\mu}_{n}}$, onde $C_{\dot{\mu}_{n}}$ é definido como no Lema 5.9. Pelos Lemas 1.28 e $5.9 C$ é fechado e ilimitado em $\omega_{1}$. Tome $\alpha \in C$ tal que, para todo $n \in \omega,\left\|\left.\mu_{n}\right|_{\mathcal{B}_{\alpha}}\right\|=\left\|\mu_{n}\right\|$. Para mostrar que existe tal $\alpha$, considere, para cada par de naturais $(n, m)$ um $a_{\left(\mu_{n}, m\right)} \in \mathcal{B}$ tal que $\left|\mu_{n}\left(a_{\left(\mu_{n}, m\right)}\right)\right|>|| \mu_{n} \|-\frac{1}{m}$. Seja $\beta_{\left(\mu_{n}, m\right)}$ tal que $a_{\left(\mu_{n}, m\right)} \in \mathcal{B}_{\beta_{\left(\mu_{n}, m\right)}}$. Tomando $\beta$ o supremo de todos $\beta_{\left(\mu_{n}, m\right)}$ achamos $\alpha>\beta, \operatorname{supp}(p)$ pertencente a $C$.

Também podemos assumir que $\left\{\left.\mu_{n}\right|_{\mathcal{B}_{\alpha}}: n \in \omega\right\}$ não é fracamente relativamente compacto em $M\left(\bar{K}_{\alpha}\right)$, tomando $\alpha$ suficiente para conter uma seqüência $W_{n}$ em $\mathcal{B}$ tal que $\left|\mu_{n}\left(W_{n}\right)\right|>\varrho$, para algum $\varrho>0$. Da mesma forma, usando a Proposição 4.4 e que $\left(\mu_{n}\right)_{n \in \omega}$ são duas a duas disjuntas, assumimos que existem $\left(A_{n}\right)_{n \in \omega} \subseteq \mathcal{B}_{\alpha}$ dois a dois disjuntos e tais que ||$\mu_{n}||-\left|\mu_{n}\right|\left(A_{n}\right)<\frac{\varrho}{90}\left\|\mu_{n}\right\|$. Assumimos ainda que

$$
\begin{gathered}
\left.T^{*}\left(\delta_{x_{n}}\right)\right|_{\mathcal{B}_{\alpha}}\left(\left\{\left.x_{n}\right|_{\alpha}\right\}\right)=T^{*}\left(\delta_{x_{n}}\right)\left(\left\{x_{n}\right\}\right), \\
\left.f \delta_{x_{n}}\right|_{\mathcal{B}_{\alpha}}\left(\left\{\left.x_{n}\right|_{\alpha}\right\}\right)=f \delta_{x_{n}}\left(\left\{x_{n}\right\}\right)
\end{gathered}
$$

e

$$
\left.\lambda_{n}\right|_{\mathcal{B}_{\alpha}}\left(\left\{\left.x_{n}\right|_{\alpha}\right\}\right)=\lambda_{n}\left(\left\{x_{n}\right\}\right) .
$$

Para isso, usando regularidade das medidas, tomamos $a_{n, m}, b_{n, m}, c_{n, m} \in \mathcal{B}$ contendo $x_{n}$ tais que

$$
\begin{gathered}
\left|T^{*}\left(\delta_{x_{n}}\right)\left(a_{n, m}\right)-T^{*}\left(\delta_{x_{n}}\right)\left(\left\{x_{n}\right\}\right)\right|<\frac{1}{m}, \\
\left|f \delta_{x_{n}}\left(b_{n, m}\right)-f \delta_{x_{n}}\left(\left\{x_{n}\right\}\right)\right|<\frac{1}{m}, \\
\left|\lambda_{n}\left(c_{n, m}\right)-\lambda_{n}\left(\left\{x_{n}\right\}\right)\right|<\frac{1}{m}
\end{gathered}
$$

e tomamos $\alpha$ suficientemente grande para que $\mathcal{B}_{\alpha}$ contenha todos $a_{n, m}$ 's, $b_{n, m}$ 's e $c_{n, m}$ 's. 
Observem que $\left.\mu_{n}\right|_{\mathcal{B}_{\alpha}}\left(\left\{\left.x_{n}\right|_{\alpha}\right\}\right) \stackrel{n}{\longrightarrow} 0$, pois

$$
\begin{gathered}
\left.\mu_{n}\right|_{\mathcal{B}_{\alpha}}\left(\left\{\left.x_{n}\right|_{\alpha}\right\}\right)=\mu_{n}\left(\left\{x_{n}\right\}\right)=T^{*}\left(\delta_{x_{n}}\right)\left(\left\{x_{n}\right\}\right)-f \delta_{x_{n}}\left(\left\{x_{n}\right\}\right)+\lambda_{n}\left(\left\{x_{n}\right\}\right)= \\
T^{*}\left(\delta_{x_{n}}\right)\left(\left\{x_{n}\right\}\right)-\int_{\left\{x_{n}\right\}} f \delta_{x_{n}}+\lambda_{n}\left(\left\{x_{n}\right\}\right)= \\
T^{*}\left(\delta_{x_{n}}\right)\left(\left\{x_{n}\right\}\right)-f\left(x_{n}\right)+\lambda_{n}\left(\left\{x_{n}\right\}\right)=\lambda_{n}\left(\left\{x_{n}\right\}\right),
\end{gathered}
$$

que converge a 0 , pois, pelo Teorema de Dieudonné-Grothendieck, para toda seqüência $\left(v_{n}\right)_{n \in \omega}$ de vizinhanças duas a duas disjuntas de $x_{n}, \lambda_{n}\left(V_{n}\right)$ converge a 0 .

Trabalhando em $M_{\alpha}$, como $K_{\alpha}$ tem peso enumerável, $C\left(K_{\alpha}\right)$ é separável. Logo, $B_{C\left(K_{\alpha}\right)^{*}}$, a bola unitária de $C\left(K_{\alpha}\right)^{*}$, com a topologia fraca* é metrizável (Proposição 3.24 de [Fa]). Como, pelo Teorema de Alaoglu, $B_{C\left(K_{\alpha}\right)^{*}}$ é compacto na topologia fraca*, podemos assumir, passando a uma subseqüência, que $\left|\mu_{n \mid \mathcal{B}_{\alpha}}\right|$ converge fracamente*, em $M\left(K_{\alpha}\right)$. Seja $\mu$ o limite fraco* de $\left|\mu_{n \mid \mathcal{B}_{\alpha}}\right|$.

Passando a uma subseqüência assumimos que $\left(\left.x_{n}\right|_{\alpha}\right)_{n \in \omega}$ é uma seqüência convergente.

Seja $\varepsilon=\frac{\varrho}{5}$. Tome $q \in P$ definido por $q(\alpha)=(0, \emptyset,\{\mu\}, \varepsilon, \emptyset)$ e $q(\beta)=p(\beta)$ para $\beta \neq \alpha$.

Tome $r \leq q$ de modo a satisfazer as hipóteses do Lema 5.10. Defina

$$
f_{\alpha}=\tilde{f}_{G_{\{\alpha\}}}^{\prime} \circ \pi_{\alpha+1}
$$

e seja $\dot{f}_{\alpha}$ um $P$-nome para $f_{\alpha}$. Como $f_{\alpha}\left(x_{n}\right)=f_{G_{\{\alpha\}}}\left(\left.x_{n}\right|_{\alpha}\right)$, para todo $n$, usando o Lema 5.10 obtemos $\delta_{1}>\delta_{2}>0$ tais que

$$
\begin{gathered}
r \Vdash \forall k \exists n_{1}, n_{2}>k\left|\int \dot{f}_{\alpha} d \dot{\mu}_{n_{1}}\right|>\delta_{1}>\delta_{2}>\left|\int \dot{f}_{\alpha} d \dot{\mu}_{n_{2}}\right|, \\
\dot{x}_{n_{1}}, \dot{x}_{n_{2}} \in \Omega_{G_{\{\alpha\}},}\left|\dot{f}_{\alpha}\left(\dot{x}_{n_{1}}\right)-\dot{f}_{\alpha}\left(\dot{x}_{n_{2}}\right)\right|<\frac{2}{k} .
\end{gathered}
$$

Encontramos, então, subconjuntos infinitos disjuntos $a_{\alpha}, b_{\alpha} \in M_{\alpha}\left[G_{\alpha}\right]$ de $\omega$ tais que $\left|\int f_{\alpha} d \mu_{n}\right|>\delta_{1}$, para $n \in a_{\alpha}$, e $\left|\int f_{\alpha} d \mu_{n}\right|<\delta_{2}$, para $n \in b_{\alpha}$, e

$$
\lim _{n \in a_{\alpha}} f_{\alpha}\left(x_{n}\right)=\lim _{n \in b_{\alpha}} f_{\alpha}\left(x_{n}\right)
$$

Chamaremos o limite acima de $L^{\prime}$. 
Como $\lambda_{n}$ converge fracamente a $\lambda, \int f_{\alpha} d \lambda_{n} \stackrel{n}{\longrightarrow} \int f_{\alpha} d \lambda$. Refinando $a_{\alpha} \mathrm{e}$ $b_{\alpha}$ podemos assumir que, para todo $n \in a_{\alpha} \cup b_{\alpha}$,

$$
\left|\int f_{\alpha} d \lambda_{n}-\int f_{\alpha} d \lambda\right|<\frac{\delta_{1}-\delta_{2}}{8} .
$$

Refinando novamente $a_{\alpha}$ e $b_{\alpha}$, podemos assumir que, para todo $n \in a_{\alpha} \cup b_{\alpha}$,

$$
\left|f\left(x_{n}\right)-L\right|<\frac{\delta_{1}-\delta_{2}}{8}
$$

lembrando que $L$ é o limite de $f\left(x_{n}\right)$. Como $\left(\left.x_{n}\right|_{\alpha+1}\right)_{n \in a_{\alpha}}$ e $\left(\left.x_{n}\right|_{\alpha+1}\right)_{n \in b_{\alpha}}$ convergem a $(z, L)$, onde $z$ é o limite de $\left(\left.x_{n}\right|_{\alpha}\right)_{n \in \omega}$, temos que

$$
\overline{\left\{\left.x_{n}\right|_{\alpha+1}: n \in a_{\alpha}\right\}} \cap \overline{\left\{\left.x_{n}\right|_{\alpha+1}: n \in b_{\alpha}\right\}} \neq \emptyset
$$

em $K_{\alpha+1}$. Como $a_{\alpha}, b_{\alpha} \in M_{\alpha}\left[G_{\alpha}\right]=M_{\alpha+1}$ e $\left\{x_{n}: n \in \omega\right\} \subseteq\left\{q_{m}: m \in \omega\right\}$, pelo Lema 5.11 temos

$$
\overline{\left\{x_{n}: n \in a_{\alpha}\right\}} \cap \overline{\left\{x_{n}: n \in b_{\alpha}\right\}} \neq \emptyset,
$$

em $K$.

Mas, usando que $T\left(f_{\alpha}\right)\left(x_{n}\right)=\int f_{\alpha} d T^{*}\left(\left\{\delta_{x_{n}}\right\}\right)$ e $f \delta_{x_{n}}\left(f_{\alpha}\right)=f\left(x_{n}\right)$, tomando

$$
U=\left\{x \in \mathbb{R}:\left|x-L-\int f_{\alpha} d \lambda\right|>\delta_{1}-\frac{\delta_{1}-\delta_{2}}{4}\right\}
$$

e

$$
V=\left\{x \in \mathbb{R}:\left|x-L-\int f_{\alpha} d \lambda\right|<\delta_{2}+\frac{\delta_{1}-\delta_{2}}{4}\right\}
$$

temos que $U$ e $V$ são abertos disjuntos de $\mathbb{R}$ e

$$
T\left(f_{\alpha}\right)\left(x_{n}\right)=\int f_{\alpha} d \mu_{n}+f\left(x_{n}\right)+\int f_{\alpha} d \lambda_{n} \in U
$$

para $n \in a_{\alpha} \mathrm{e}$

$$
T\left(f_{\alpha}\right)\left(x_{n}\right)=\int f_{\alpha} d \mu_{n}+f\left(x_{n}\right)+\int f_{\alpha} d \lambda_{n} \in V
$$

para $n \in b_{\alpha}$, o que daria, pela continuidade de $T\left(f_{\alpha}\right)$, que $\overline{\left\{x_{n}: n \in a_{\alpha}\right\}}$ e $\overline{\left\{x_{n}: n \in b_{\alpha}\right\}}$ são disjuntos, chegando numa contradição.

Teorema 5.14. É consistente com ZFC que existe um espaço de Banach $C(K)$ de densidade $\omega_{1}<2^{\omega}$ indecomponível tal que todo operador em $C(K)$ é da forma $g I+S$, para $g \in C(K)$ e $S$ fracamente compacto. 
Demonstração: Suponha $\neg C H$ no modelo inicial $M$. Como $P$ é c.c.c., $P$ preserva cardinais e, como $\left(2^{\omega}\right)^{M} \leq\left(2^{\omega}\right)^{M[G]}, \neg C H$ é preservado em $M[G]$. Por ser um subespaço de $[0,1]^{\omega_{1}}, K_{\omega_{1}}$ tem peso $\omega_{1}$ em $M[G]$ e, portanto, a densidade de $C\left(K_{\omega_{1}}\right)$ é $\omega_{1}<2^{\omega}$. Dos Lemas $5.13,5.12$ e 1.10 e do Teorema 1.11 segue que todo operador em $C\left(K_{\omega_{1}}\right)$ é da forma $g I+S$, para $g \in C\left(K_{\omega_{1}}\right)$ e $S$ fracamente compacto. Pela conexidade de $K_{\omega_{1}}$ (Lema 5.8) e pelo Lema 1.12 segue que $C\left(K_{\omega_{1}}\right)$ é indecomponível. 


\section{Apêndice A}

\section{Representação de Stone}

Nos Capítulos 3 e 4 construímos espaços compactos 0-dimensionais como espaços de Stone de álgebras de Boole. Neste apêndice iremos descrever a dualidade de Stone, que associa biunivocamente álgebras de Boole com espaços booleanos, isto é, os espaços compactos totalmente desconexos.

\section{A.1 Álgebras de Boole}

Definição A.1. Uma álgebra de Boole é uma estrutura $\mathcal{A}=(A, \wedge, \vee,-, 0,1)$, onde $\wedge$ e $\vee$ são operações binárias em $A$, - é uma operação unária e 0 e 1 são dois elementos distintos de $A$, que satisfaz, para todo $x, y, z \in A$ :

$B 1 x \vee(y \vee z)=(x \vee y) \vee z ;($ associatividade)

$B 1^{\prime} x \wedge(y \wedge z)=(x \wedge y) \wedge z$

$B 2 x \vee y=y \vee x ;$ (comutatividade)

$B 2^{\prime} x \wedge y=y \wedge x$

$B 3 x \vee(x \wedge y)=x ;($ absorção)

$B 3^{\prime} x \wedge(x \vee y)=x$;

$B 4 x \wedge(y \vee z)=(x \wedge y) \vee(x \wedge z) ;($ distributividade)

$B 4^{\prime} x \vee(y \wedge z)=(x \vee y) \wedge(x \vee z)$;

$B 5 x \vee(-x)=1 ;($ complementação) 
$B 5^{\prime} x \wedge(-x)=0$.

Dada uma álgebra de Boole $\mathcal{A}=(A, \wedge, \vee,-, 0,1)$, chamamos o conjunto $A$ de domínio de $\mathcal{A}$. Por abuso de notação, eventualmente denotaremos a álgebra $\mathcal{A}$ pelo seu domínio $A$.

Definição A.2. Dadas duas álgebras de Boole $\mathcal{A}$ e $\mathcal{B}$, de domínios $A$ e $B$, respectivamente, um homomorfismo entre $\mathcal{A}$ e $\mathcal{B}$ é uma função $h: A \longrightarrow B$ satisfazendo

$$
h(0)=0, h(1)=1
$$

e, para todo $x, y \in A$,

$$
h(x \wedge y)=h(x) \wedge h(y), h(x \vee y)=h(x) \vee h(y), h(-x)=-h(x) .
$$

Um isomorfismo é um homomorfismo bijetor. Denotamos por $\mathcal{A} \cong \mathcal{B}$ quando $\mathcal{A}$ é isomorfo a $\mathcal{B}$.

Definição A.3. Numa álgebra de Boole $A$, definimos a relação $\leq$ por $x \leq y$ se e somente se $x=x \vee y$, para todo $x, y \in A$.

\section{Álgebras de Conjuntos}

Definição A.4. Uma álgebra de conjuntos sobre $X$, que também chamaremos de corpo de conjuntos sobre $X$ é uma família $\mathcal{A} \subseteq \mathcal{P}(X)$ que contém $X$ e é fechado por intersecções finitas e complementos, isto é:

(a) $X \in \mathcal{A}$;

(b) Se $Y, Z \in \mathcal{A}$ então $Y \cap Z \in \mathcal{A}$;

(c) Se $Y \in \mathcal{A}$ então $X \backslash Y \in \mathcal{A}$.

Dessas três propriedades, deduzimos as seguintes:

(d) $\emptyset \in \mathcal{A}$;

(e) Se $Y, Z \in \mathcal{A}$ então $Y \cup Z \in \mathcal{A}$.

O próximo lema demonstra-se através de uma simples verificação dos axiomas de álgebras de Boole. 
Lema A.5. Uma álgebra de conjuntos sobre $X$ é uma álgebra de Boole, onde $0=\emptyset, 1=X$ e as operações $\wedge, \vee e-$ são, respectivamente, intersecção, união e complemento em relação a $X$.

A ordem em uma álgebra de conjuntos, conforme a Definição A.3, coincide com a ordem da inclusão. Veremos, pela dualidade de Stone, que toda álgebra de Boole é isomorfa a uma álgebra de conjuntos.

\section{A.2 Teorema da Representação de Stone}

Nesta seção iremos descrever a representação de Stone. Para as demonstrações dos resultados que constam nesta seção indicamos [Kop].

Definição A.6. Seja $A$ uma álgebra de Boole. Dizemos que $F \subseteq A$ é um filtro em $A$ se

- $1 \in F$;

- $0 \notin F$;

- Se $x, y \in F$ então $x \wedge y \in F$;

- Para todo $x, y \in A$, se $x \in F$ e $x \leq y$, então $y \in F$.

Um ultrafiltro em $A$ é um filtro maximal, isto é, um filtro que não está contido propriamente em nenhum filtro.

Definição A.7. Seja $A$ uma álgebra de Boole. Dizemos que $F$ tem a propriedade da intersecção finita (p.i.f.) se para todos $x_{1}, \ldots, x_{n} \in F$ temos $x_{1} \wedge \ldots \wedge x_{n} \neq 0$.

Lema A.8. Seja A uma álgebra de Boole.

(a) Se $F \subseteq A$ tem a propriedade da intersecção finita então existe um ultrafiltro que contém $F$;

(b) Um filtro $F$ em A é um ultrafiltro se e somente se para todo $a \in A$, ou $a \in F$ ou $-a \in F$;

(c) Dados $a, b \in A$ distintos, existe um ultrafiltro $u$ em $A$ tal que $a \in u e$ $b \notin u$, ou $b \in u$ e $a \notin u$. 
Definição A.9. Dado $K$ um espaço topológico compacto 0-dimensional, definimos $C l o p(K)$ a álgebra de conjuntos formado pelos abertos-fechados de $K$. Dada uma álgebra de Boole $A$, definimos o espaço de Stone de $A$, denotado $S(A)$, como o conjunto dos ultrafiltros em $A$ munido da topologia gerada por $\left\{a^{*}: a \in A\right\}$, onde $a^{*}=\{u \in S(A): a \in u\}$.

Teorema A.10 (Stone). Seja A uma álgebra de Boole. Temos $A \cong C l o p(S(A))$ pelo isomorfismo $h: A \longrightarrow C l o p(S(A))$ dado por $h(a)=a^{*}$. Além disso, para todo compacto 0-dimensional $K$ temos $K$ homeomorfo a $S(C l o p(K))$.

Da Definição A.9 segue imediatamente o seguinte Lema:

Lema A.11. Se A é uma álgebra de Boole então $S(A)$ tem peso $|A|$.

Definição A.12. Definimos $\beta \mathbb{N}$ o espaço de Stone da álgebra de conjuntos $\mathcal{P}(\omega)$

Dizemos que um espaço topológico $X$ é $T_{3 \frac{1}{2}}$ se para todo $x \in X$ e $F \subseteq$ $X \backslash\{x\}$ fechado existe uma função contínua $f: X \longrightarrow \mathbb{R}$ tal que $\left.f\right|_{F}=0$ e $f(x)=1$. Se $X$ é um espaço topológico $T_{3 \frac{1}{2}}$ definimos o compactificado de Stone-Cech de $X$ como o menor compacto $\beta X$ tal que toda a função contínua limitada de $X$ em $\mathbb{R}$ se estende de forma única como uma função contínua de $\beta X$ em $\mathbb{R}$. A existência e unicidade, a menos de homeomorfismos, do compactificado de Stone-Čech para espaços $T_{3 \frac{1}{2}}$ pode ser vista em [Eng].

Teorema A.13. O espaço topológico $\beta \mathbb{N}$ é o compactificado de Stone-Čech dos naturais com a topologia discreta. 


\section{Apêndice B}

\section{Forcing}

Neste apêndice faremos uma breve explicação sobre o método Forcing, para auxiliar a compreensão dos Capítulos 4 e 5.

Seja $\Gamma$ um conjunto de fórmulas. Dizemos que $\Gamma$ é consistente se dele não podemos deduzir uma contradição. Ou seja, se $\Gamma \nvdash \varphi \wedge \neg \varphi$. Dizemos que uma fórmula $\varphi$ é relativamente consistente com $\Gamma$ se a consistência de $\Gamma$ implica a consistência de $\Gamma \cup\{\varphi\}$, isto é, se $\varphi$ não pode ser deduzido de $\Gamma$, a menos que $\Gamma$ seja inconsistente (nesse caso, podemos deduzir dele qualquer fórmula). Usamos a notação $\operatorname{Con}(\Gamma) \longrightarrow \operatorname{Con}(\Gamma+\varphi)$, para denotar a consistência relativa de $\varphi$ com $\Gamma$. Dizemos que $\varphi$ é independente de $\Gamma$ se tanto $\varphi$ quanto $\neg \varphi$ são relativamente consistente com $\Gamma$.

O forcing foi criado em 1964 pelo matemático Paul Cohen para mostrar a consistência da negação da Hipótese do Contínuo, resultado que lhe concedeu a medalha Fields, em 1966. A consistência da Hipótese do Contínuo havia sido mostrada por Kurt Gödel, na década de 30. Inúmeros resultados de independência têm sido demonstrados através de forcing em diversas áreas da matemática, como teoria dos conjuntos, topologia e análise funcional.

\section{B.1 Modelos transitivos enumeráveis}

Iremos descrever sucintamente a sintaxe e semântica da lógica clássica.

A linguagem da teoria dos conjuntos é formada pelos seguintes símbolos: os quantificadores $\forall$ (para todo) e $\exists$ (existe), os conectivos $\neg$ (não), $\wedge$ (e), $\vee(\mathrm{ou}), \rightarrow$ (se, então) e $\leftrightarrow$ (se, e somente se), a igualdade $=$, a relação de pertinência $\in$, os delimitadores ( $\mathrm{e}$ ), e as variáveis $x_{1}, x_{2}, x_{3}, \ldots$ 
Uma fórmula atômica é uma seqüência de símbolos $(x=y)$ ou $(x \in y)$, onde $x$ e $y$ são variáveis. Uma fórmula é uma fórmula atômica ou uma seqüência de símbolos da forma $\neg \phi, \forall x \phi, \exists x \phi,(\phi \wedge \psi),(\phi \vee \psi),(\phi \rightarrow \psi)$ ou $(\phi \leftrightarrow \psi)$, onde $x$ é uma variável e $\phi$ e $\psi$ são fórmulas. Nesses casos, $\phi$ e $\psi$ são subfórmulas das fórmulas anteriores.

Uma variável livre de uma fórmula é uma variável $x$ que ocorre em algum lugar fora do escopo de um quantificador, isto é, nem todas ocorrências de $x$ está em alguma subfórmula do tipo $\forall x \phi$ ou $\exists x \phi$. A notação $\phi\left(x_{1}, \ldots, x_{n}\right)$ indica que as variáveis livres de $\phi$ estão contidas em $\left\{x_{1}, \ldots, x_{n}\right\}$.Uma sentença é uma fórmula sem variáveis livres.

Um modelo para teoria dos conjuntos é um par $(M, \varepsilon)$, onde $M$ é um conjunto e $\varepsilon \subseteq M \times M$ é uma relação binária. Usaremos a notação $x \varepsilon y$ para $(x, y) \in \varepsilon$.

Dizemos que um modelo $(M, \varepsilon)$ é standard se a relação $\varepsilon$ coincide com a relação $\in$, isto é, $\varepsilon=\{(x, y) \in M: x \in y\}$. Para modelos standard, denotamos $(M, \in)$ simplesmente por $M$. Um modelo standard $M$ é dito transitivo se $x \subseteq M$, para todo $x \in M$.

Iremos trabalhar apenas com modelos standard.

Definição B.1. ([Ku], IV, 2.1) Seja $M$ uma classe. Para cada fórmula $\phi$ definimos a relativização de $\phi$ a $M$, que será denotada por $\phi^{M}$, do seguinte modo:

1. $(x=y)^{M}$ é $x=y$;

2. $(x \in y)^{M}$ é $x \in y$;

3. $(\phi \wedge \psi)^{M}$ é $\phi^{M} \wedge \psi^{M}$;

4. $(\neg \phi)^{M}$ é $\neg\left(\phi^{M}\right)$;

5. $(\exists x \phi)^{M}$ é $\exists x\left(x \in M \wedge \phi^{M}\right)$.

Dizemos que $M$ satisfaz $\phi$, e denotaremos por $M \models \phi$, se $\varphi^{M}$ vale.

Teorema B.2. ([Ku], IV, 7.11) Se $\Gamma$ é um conjunto finito consistente de fórmulas, existe um modelo transitivo enumerável $M$ tal que $M \models \Gamma$. 
Para toda a matemática, usamos o sistema de axiomas ZFC (sistema de Zermelo-Frankel com Axioma da Escolha), formado por infinitos axiomas ${ }^{1}$.

Não podemos provar, em ZFC, que existe um modelo para ZFC, pois isso provaria a consistência de ZFC e, pelo Teorema da Incompletude de Gödel, um sistema de axiomas não pode provar sua própria consistência. Portanto, não podemos provar, em ZFC, que existe um modelo para ZFC.

Dizemos que uma fórmula $\varphi$ é relativamente consistente com um conjunto $\Gamma$ se a consistência de $\Gamma$ implica a consistência de $\Gamma \cup\{\varphi\}$.

De modo geral, quando estiver claro o contexto, dizemos simplesmente que uma fórmula é consistente se é relativamente consistente com ZFC.

Para mostrar a consistência de uma fórmula usando forcing precisamos assumir a existência de um modelo transitivo enumerável. Como ZFC não é finitamente axiomatizável, mesmo se ZFC for consistente pode não existir um modelo transitivo para ZFC. Porém, como demonstrações usam finitas fórmulas, do Teorema B.2 concluímos o seguinte resultado (veja demonstração em [Ku], VII §1), que também é conhecido como princípio do forcing (vide $[\mathrm{Ci}]$ ).

Teorema B.3 (Princípio do forcing). Para mostrar que uma fórmula $\varphi$ é relativamente consistente com ZFC é suficiente mostrar que, para todo modelo transitivo enumerável $M$ que satisfaz $Z F C$, existe um modelo $N$ que satisfaz $Z F C$ tal que $M \subseteq N$ e $N \models \varphi$.

\section{B.2 Ordens parciais e filtros genéricos}

Uma ordem parcial é um par $(P, \leq)$ onde $\leq$ contido em $P \times P$ é uma relação binária reflexiva $(x \leq x)$, transitiva $(x \leq y \wedge y \leq z \rightarrow x \leq z)$ e anti-simétrica $(x \leq y \wedge y \leq x \rightarrow x=x)$. Chamaremos de forcing uma ordem parcial $(P, \leq)$ com um elemento máximo, que será denotado por $\mathbf{1}_{P}$. Os elementos de $P$ serão chamados de condições do forcing. Uma condição $p \in P$ é dita mais forte que $q \in P$ quando $p \leq q$. Duas condições, $p$ e $q$ são compatíveis se existe $r \in P$ tal que $r \leq p$ e $r \leq q$. Denotamos por $p \perp q$ quando $p$ e $q$ são incompatíveis.

Dizemos que um forcing $P$ é trivial se existe $p \in P$ tal que, para todos $q, r \leq p, q$ e $r$ são compatíveis.

\footnotetext{
${ }^{1} \mathrm{Na}$ prática, o ZFC é apresentado com nove axiomas. Mas um deles, o Axioma da Substituição, é, na verdade, uma lista infinita enumerável de axiomas, onde, para cada fórmula, temos um axioma correspondente.
} 
Um subconjunto $D \subseteq P$ é denso em $P$ se para todo $p \in P$ existe $q \in D$ tal que $q \leq p$. Um subconjunto $A \subseteq P$ é uma anticadeia em $P$ se os elementos de $A$ são dois a dois incompatíveis.

Dizemos que $G \subseteq P$ é um filtro em $P$ se $p$ e $q$ são compatíveis, para todos $p, q \in G$. Dizemos que $G$ é um filtro P-genérico (ou, simplesmente, $P$-genérico), se $G$ é um filtro que intercepta todos densos em $P$. Se $M$ é um modelo transitivo enumerável para ZFC e $P \in M$, dizemos que um subconjunto $G \subseteq P$ é $P$-genérico sobre $M$ se $G$ é um filtro e, para todo $D \in M$ denso em $P$, temos $G \cap D \neq \emptyset$.

Lema B.4. ([Ku], VII, 2.4) Um forcing $P$ é trivial se, e somente se, existe um P-genérico.

Lema B.5. ([Ku], VII, 2.3) Sejam $M$ um modelo transitivo enumerável para $Z F C$ e $P \in M$ um forcing. Então, para todo $p \in P$ existe $G$ um filtro $P$-genérico sobre $M$ tal que $p \in G$.

Para mostrar a consistência de uma sentença, usando forcing, tomamos $M$ um modelo transitivo enumerável para ZFC (que podemos assumir que existe, pelo princípio do forcing) e um forcing não-trivial $P \in M$. Como $P$ é não-trivial, pelo Lema B.4 não existe, em $M$, um $P$-genérico sobre $M$. Mas, pelo Lema B.5, fora de $M$ existe um $P$-genérico sobre $M$. Nosso objetivo é adicionar a $M$ um $P$-genérico sobre $M$. Isto é, dado $G$ um $P$-genérico sobre $M$ encontramos $M[G]$ o menor modelo transitivo enumerável tal que $M[G] \models Z F C, M \subseteq M[G]$ e $G \in M[G]$. Esse método é semelhante ao de extensões de corpos, usado na Teoria de Galois. Dado um corpo $\mathbb{K}$ (por exemplo, os racionais) achamos o menor corpo $K(\sqrt{2})$ que contenha $K$ e a equação $x^{2}-2=0$ tenha solução. Da mesma forma, o genérico $G$ será adicionado a $M$ para "forçar" uma fórmula $\varphi$.

Como exemplo, o forcing de Cohen, usado para mostrar a consistência de $\neg C H$, é o conjunto de todas as funções finitas de um conjunto $X$ em $\{0,1\}$. A ordem é dada por $f \leq g$ se, e somente se, $\operatorname{supp}(f) \supseteq \operatorname{supp}(g)$ e $\left.f\right|_{\operatorname{supp}(g)}=$ $g$. Se $G$ é um filtro genérico, $\bigcup G$ é uma função total de $X$ em $\{0,1\}$ (a compatibilidade dos elementos de $G$ garantem que $\bigcup G$ é uma função, e por interceptar todos os densos de $M$ é fácil concluir que $\operatorname{dom}(\bigcup G)=X)$. Observamos que o forcing de Cohen é trivial se, e somente se, $X$ é finito. 


\section{B.3 Extensões genéricas}

Seja $P$ um forcing. Dizemos que $\tau$ é um $P$-nome se é um conjunto de pares ordenados da forma $(\sigma, p)$, onde $p \in P$ e $\sigma$ é um $P$-nome ${ }^{2}$. Denotamos por $V^{P}$ a classe de todos os $P$-nomes ${ }^{3}$.

Sejam $M$ um modelo transitivo enumerável e $P \in M$ um forcing nãotrivial. Dizemos que $\tau$ é um $P$-nome sobre $M$ se $\tau \in M$ é um $P$-nome ${ }^{4}$. Definimos $M^{P}=M \cap V^{P}$ o conjunto de todos os $P$-nomes sobre $M$. Se $G$ é um $P$-genérico sobre $M$ e $\tau$ é um $P$-nome sobre $M$, definimos $\tau_{G}=\left\{\sigma_{G}\right.$ : $\exists p \in G(\sigma, p) \in \tau\}$. Definimos

$$
M[G]=\left\{\tau_{G}: \tau \in M^{P}\right\} .
$$

Para $x \in V($ ou $x \in M)$, definimos um $P$-nome (sobre $M) \check{x}=\{\check{y}: y \in$ $x\}$. Definimos um $P$-nome $\Gamma=\{(\check{p}, p): p \in P\}$.

Lema B.6. ([Ku], VII, 2.11 e 2.13) Sejam $M$ um modelo transitivo enumerável para $Z F C, P \in M$ um forcing e $G$ um P-genérico sobre $M$. Então

a) $\check{x}_{G}=x$, para todo $x \in M$;

b) $\Gamma_{G}=G$.

O próximo teorema é fundamental no método do forcing.

Teorema B.7. ([Ku], VII, 2.14, 4.2, 2.11, 2.13, 2.19) Sejam $M$ um modelo transitivo enumerável para $Z F C, P \in M$ um forcing e $G$ um $P$ genérico sobre $M$. Então

\footnotetext{
${ }^{2}$ Para formalizar a boa definição de $P$-nomes, assim como outras definições apresentadas nesta seção, usamos o Teorema da recursão sobre relações bem fundadas (vide $[\mathrm{Ku}]$ ). Intuitivamente, funciona como uma definição recursiva sobre $\omega$, ou sobre um ordinal qualquer. O conjunto vazio é um $P$-nome. Portanto $\{(\emptyset, p): p \in P\}$ também é um $P$-nome, bem como $\{((\emptyset, p), q): p, q \in P\}$, e assim por diante

${ }^{3}$ Usamos a noção informal de classes. A cada fórmula de uma variável livre associamos a classe dos conjuntos que satisfazem tal fórmula. Por exemplo, a fórmula $x=x$ nos fornece a classe $V$ de todos os conjuntos. A classe $V^{P}$ é formada de todos os conjuntos $x$ tal que $x$ é um $P$-nome. Pelo Paradoxo de Russel, sabemos que a classe de todos os conjuntos não é um conjunto. É fácil verificar que $V^{P}$ não é um conjunto, pois poderíamos obter dele $V$ como conjunto. A uma classe que não é conjunto chamamos classe própria. Para formalizar a noção de classes, temos as teorias NGB (von Neumann-Bernays-Gödel), e MK (Morse-Kelley, onde as classes são apresentadas em vários níveis hierárquicos). Em ZFC, trabalhamos com a noção de classes como uma notação. Assim escrevemos $\tau \in V^{P}$ para dizermos que " $\tau$ é um $P$-nome".

${ }^{4}$ Equivalentemente, se $M \models$ " $\tau$ é um $P$-nome", pois o conceito de $P$-nomes é absoluto.
} 
a) $M[G]$ é um modelo transitivo enumerável;

b) $M[G] \models Z F C$;

c) $M \subseteq M[G]$;

d) $G \in M$;

e) Se $N \supseteq M$ satisfaz os itens anteriores, então $M[G] \subseteq N$.

Notemos que os itens $c$ ) e $d$ ) são conseqüências do Lema B.6. Item $e$ ) segue do fato que, se $G \in N, M \subseteq N$ e $N \models Z F C$, então podemos definir $\tau_{G}$ em $N$, para todo $\tau \in M^{P}$. Item $\left.a\right)$ é facilmente verificado pela definição de $M[G]$. A dificuldade da demonstração do Teorema B.7 está no item $b$ ).

\section{B.4 Relação de forcing}

Sejam $M$ um modelo transitivo enumerável para ZFC e $P \in M$ um forcing. Definimos a linguagem do forcing $P$ sobre $M$, que denotaremos por $\mathcal{L}\left(M^{P}\right)$, como a linguagem da teoria dos conjuntos substituindo as variáveis livres por $P$-nomes em $M$.

Teorema B.8. ([Je], 14.6 e 14.7) Sejam $M$ um modelo transitivo enumerável para $Z F C$ e $P \in M$ um forcing. Existe uma relação $\Vdash \subseteq P \times \mathcal{L}\left(M^{P}\right)$ tal que

(i) $\Vdash \in M$;

(ii) (a) $p \Vdash \varphi\left(\tau_{1}, \ldots, \tau_{n}\right)$ se, e somente se, $M[G] \models \varphi\left(\left(\tau_{1}\right)_{G}, \ldots,\left(\tau_{n}\right)_{G}\right)$, para todo $G$ filtro P-genérico sobre $M$ tal que $p \in G$;

(b) $M[G] \models \varphi\left(\left(\tau_{1}\right)_{G}, \ldots,\left(\tau_{n}\right)_{G}\right)$ se, e somente se, existe $p \in G$ tal que $p \Vdash \varphi\left(\tau_{1}, \ldots, \tau_{n}\right) ;$

(iii) (a) Se $p \Vdash \varphi$ e $q \leq p$ então $q \Vdash \varphi$;

(b) Não existe $p \in P$ e $\varphi \in \mathcal{L}\left(M^{P}\right)$ tal que $p \Vdash \varphi$ e $p \Vdash \neg \varphi$;

(c) Para todos $p \in P$ e $\varphi \in \mathcal{L}\left(M^{P}\right)$ existe $q \leq p$ tal que $q \Vdash \varphi$ ou $q \Vdash \neg \varphi ;$

(d) $p \Vdash \neg \varphi$ se, e somente se, $q \Vdash \varphi$, para todo $q \leq p$;

(e) $p \Vdash \varphi \wedge \psi$ se, e somente se, $p \Vdash \varphi e p \Vdash \psi$; 
(f) $p \Vdash \exists x \varphi(x)$ se, e somente se, existe $\tau \in M^{P}$ tal que $p \Vdash \varphi(\tau)$.

(iv) $p \Vdash$ "T é um filtro" e, se $D$ é denso em $P$, então $p \Vdash \Gamma \cap \check{D} \neq \emptyset$.

Em $[\mathrm{Ku}]$ a relação $\Vdash$ está definida como no item $(i i)(a)$. No modelo inicial $M$ define-se uma relação $\Vdash^{*}$, e mostra-se que $\left(p \Vdash^{*} \varphi\right)^{M}$ se, e somente se, $p \Vdash \varphi$. Disso segue o item $(i)$. Já em [Je], define-se $\Vdash$ diretamente em $M$ (ou melhor, em ZFC), e mostra-se o item $(i i)(b)$. É fácil verificar a equivalência entre $(i i)(a)$ e $(i i)(b)$ a partir das propriedades de $(i i i)$.

O item $(i v)$ segue imediatamente do item $(i i)(a)$, da definição de genericidade e da absolutividade da intersecção (vide Seção B.5).

A relação $\Vdash$ é chamada de relação de forcing, e lemos $p \Vdash \varphi$ como $p$ força $\varphi$.

Denotamos $P \Vdash \varphi$ quando $p \Vdash \varphi$, para todo $p \in P$ (equivalentemente, quando $\left.\mathbf{1}_{P} \Vdash \varphi\right)$.

Em forcing, na maneira como aqui foi apresentada, trabalhamos com os axiomas de ZFC em três níveis: o modelo $M$, a extensão $M[G]$ e o universo $V$, a classe de todos os conjuntos.

Pelo Teorema B.8 item ( $i$ ), a relação de forcing pode ser definida no próprio modelo $M$. Por isso, uma vez desenvolvida a teoria do forcing, podemos usá-la no universo $V$, a classe de todos os conjuntos. Em $[\mathrm{Ku}]$ achamos uma definição, em $V$, da relação $\Vdash$ (de onde segue o item $a$ ), o que nos permite mostrar um resultado de independência dentro de ZFC, apenas trabalhando com a relação $\Vdash$ e usando a absolutividade de algumas fórmulas (veja Seção B.5).

\section{B.5 Absolutividade e preservação de cardi- nais}

Definição B.9. ([Ku], IV, 3.1) Sejam $\varphi$ uma fórmula com variáveis livres $x_{1}, \ldots, x_{n}$ e $M \subseteq N$ modelos transitivos para ZFC. Dizemos que

1. $\varphi$ é absoluta em relação a $M$ e $N$ se $\forall x_{1}, \ldots, x_{n} \in M\left(M \models \varphi\left(x_{1}, \ldots, x_{n}\right) \leftrightarrow\right.$ $\left.N=\varphi\left(x_{1}, \ldots, x_{n}\right)\right)$;

2. $\varphi$ é absoluta em relação a $M$ e $V$ (ou, simplesmente, absoluta em relação a $\mathrm{M})$ se $\forall x_{1}, \ldots, x_{n} \in M\left(M \models \varphi\left(x_{1}, \ldots, x_{n}\right) \leftrightarrow \varphi\left(x_{1}, \ldots, x_{n}\right)\right)$;

3. $\varphi$ é absoluta se é absoluta em relação a todo modelo transitivo. 
Lema B.10. ([Ku], IV, 3.3 e 3.7) Sejam $\varphi$ e $\psi$ fórmulas com variáveis livres contidas em $\left\{x_{1}, \ldots, x_{n}\right\}$ e $M \subseteq N$ modelos transitivos para ZFC. Então

1. Se $\varphi$ e $\psi$ são absolutas em relação a $M$ e $N$, então $\neg \varphi, \varphi \wedge \psi e$ $\exists x(x \in y \wedge \varphi)$ são absolutas em relação a $M$ e $N$;

2. Se $Z F C \vdash \forall x_{1}, \ldots, x_{n}\left(\varphi\left(x_{1}, \ldots, x_{n}\right) \leftrightarrow \psi\left(x_{1}, \ldots, x_{n}\right)\right)$ então $\varphi$ é absoluta em relação a $M$ e $N$ se, e somente se, $\psi$ o é;

3. Se $\varphi$ não contém quantificadores, então $\varphi$ é absoluta em relação a $M$ e $N$.

O Lema B.10 também vale para $V$ no lugar de $N$.

Definição B.11. ([Ku], IV, 3.5) Uma fórmula é $\Delta_{0}$ se todos seus quantificadores são limitados. Formalmente, as fórmulas $\Delta_{0}$ são definidas indutivamente pelas seguintes regras:

1. $x \in y$ e $x=y$ são $\Delta_{0}$;

2. Se $\varphi$ e $\psi$ são $\Delta_{0}$ então $\neg \varphi$ e $\varphi \wedge \psi$ são $\Delta_{0}$;

3. Se $\varphi$ é $\Delta_{0}$ então $\exists x(x \in y \wedge \varphi)$ é $\Delta_{0}$.

Do Lema B.10, item 1, segue o seguinte Corolário:

Corolário B.12. ([Ku], IV, 3.6) As fórmulas $\Delta_{0}$ são absolutas.

Quando dizemos que $\omega$ é absoluto em relação a $M$ e $N$ dizemos que a fórmula " $x$ é o primeiro ordinal infinito" é absoluta em relação a $M$ e $N$. O mesmo vale para outras fórmulas "implícitas", tais como, $\{x\},\{x, y\}, 0,2^{\omega}$, $\omega_{1}$, etc.

Lema B.13. ([Ku], IV, 5.1 e 5.3) As seguintes fórmulas são absolutas:

1. $\omega ;$

2. $x$ é um ordinal;

3. $x$ é um ordinal limite;

4. $x$ é um ordinal sucessor;

5. $x$ é finito. 
O primeiro cardinal não-enumerável, $\omega_{1}$, não é absoluto, em geral. Se $M$ é um modelo transitivo para ZFC, denotamos por $\left(\omega_{1}\right)^{M}$ o elemento $x \in M$ tal que $M \models x=\omega_{1}$. Ou seja, $\left(\omega_{1}\right)^{M}$ é a relativização a $M$ da fórmula " $x$ é o primeiro cardinal não-enumerável". A definição análoga serve para $\left(2^{\omega}\right)^{M}$, $\mathbb{R}^{M}$, etc.

Se $M \subseteq N$ são modelos transitivos para ZFC e $A \in M$ é um conjunto, então $(\mathcal{P}(A))^{M} \subseteq(\mathcal{P}(A))^{N}$, pois os subconjuntos de $A$ que pertencem a $M$ também irão pertencer a $N$, mas $N$ pode adicionar novos subconjuntos de $N$. Dessa observação segue imediatamente o seguinte lema:

Lema B.14. Sejam $M \subseteq N$ modelos transitivos para $Z F C$ e seja $\lambda$ um ordinal. Então $\left(2^{\lambda}\right)^{M} \leq\left(2^{\lambda}\right)^{N}$.

Definição B.15. ([Ku], VII, 5.7) Seja $M$ um modelo transitivo para ZFC e seja $P \in M$ um forcing. Seja $\kappa \in M$ tal que $M=$ " $\kappa$ é um cardinal". Dizemos que $P$ preserva o cardinal $\kappa$ se, para todo $\lambda<\kappa$ e $G$ filtro $P$ genérico sobre $M$, temos $M \models$ " $\lambda$ é um cardinal" se, e somente se, $M[G] \models$ " $\lambda$ é um cardinal".

Na notação descrita anteriormente, $P$ preserva um cardinal $\kappa$ se $\kappa^{M}=$ $\kappa^{M[G]}$, para todo $G$ filtro $P$-genérico sobre $M$. Dizer que $P$ preserva cardinais é o mesmo que dizer que a fórmula " $x$ é um cardinal" é absoluta em relação a $M$ e $M[G]$, para toda extensão $M[G]$ por um $P$-genérico sobre $M$.

Definição B.16. ([Ku], II, 2.3) Dizemos que um forcing $P$ possui a condição da cadeia enumerável, ou que $P$ é c.c.c. (do inglês, countable chain condition) se $P$ não contém uma anticadeia não-enumerável.

Teorema B.17. ([Ku], VII, 5.10) Se P é c.c.c. então P preserva cardinais.

\section{B.6 Forcing iterado}

Sejam $M$ um modelo transitivo enumerável, $P \in M$ um forcing e $G$ um filtro $P$-genérico sobre $M$. Suponha que temos $\dot{Q}$ um $P$-nome em $M$ tal que $P \Vdash$ "Q é um forcing". Sobre $M[G]$ podemos tomar uma extensão $M[G][H]$, onde $H$ é um $\dot{Q}_{G}$-genérico sobre $M[G]$. Através do forcing iterado, repetimos esse processo transfinitamente, sobre um ordinal $\kappa$, por meio de apenas um forcing no modelo inicial. 
Definição B.18. ([Je], 16.1 e 16.2) Sejam $M$ um modelo transitivo enumerável, $P \in M$ um forcing e $\dot{Q} \in M^{P}$ tal que $P \Vdash$ "Q $\dot{Q}$ é um forcing". Definimos $P * \dot{Q}$ a iteração de $P$ e $\dot{Q}$ o conjunto

$$
\{(p, \tau): p \in P \wedge p \Vdash \tau \in \dot{Q}\}
$$

com a ordem parcial dada por $(p, \tau) \leq(q, \sigma)$ se, e somente se,

$$
p \leq_{P} q \wedge p \Vdash \tau \leq \sigma .
$$

Se $G$ é um $P$-genérico sobre $M$ e $H$ um $\dot{Q}_{G}$-genérico sobre $M[G]$ definimos

$$
G * H=\left\{(p, \tau) \in P * \dot{Q}: p \in G \wedge \tau_{G} \in H\right\} .
$$

É fácil verificar que a iteração de forcings não-triviais é não-trivial.

Teorema B.19. ([Je], 16.2) Sejam $M$ um modelo transitivo enumerável, $P \in M$ um forcing $e \dot{Q}$ um $P$-nome para um forcing.

(a) Se $G$ é um P-genérico sobre $M$ e $H$ um $\dot{Q}_{G}$-genérico sobre $M[G]$ então $G * H$ é um $P * \dot{Q}$-genérico sobre $M$;

(b) Se K é um $P * \dot{Q}_{G}$-genérico sobre $M$, se tomarmos

$$
G=\pi_{1}(K)=\{p \in P: \exists \tau((p, \tau) \in K)\}
$$

$e$

$$
H=\left\{\tau_{G}: \exists p \in P((p, \tau) \in K)\right\}
$$

temos que $G$ é um P-genérico sobre $M, H$ é um $\dot{Q}_{G}$-genérico sobre $M[G]$ e $K=G * H$.

Definição B.20. ([Je], 16.8) Sejam $M$ um modelo transitivo enumerável e $\alpha \in M$ um ordinal. Dizmos que $P_{\alpha}$ é uma iteração de forcings de comprimento $\alpha$ com suportes finitos se $P_{\alpha}$ é um conjunto de seqüências de comprimento $\alpha$ que satisfazem

1. para $\alpha=0, P_{\alpha}$ é um forcing trivial;

2. para $\alpha=1, P_{1}$ é um forcing $Q_{0}$; 
3. para $\alpha=\beta+1>1, P_{\alpha}=P_{\beta} * \dot{Q}_{\beta}$, onde $P_{\beta}$ é uma iteração de comprimento $\beta$ com suportes finitos e $\dot{Q}_{\beta}$ é um $P_{\beta}$-nome para um forcing; a ordem em $P_{\alpha}$ é dada por $p \leq q$ se, e somente se, $p|\beta \leq q| \beta$ e $p \mid \beta \Vdash p(\beta) \leq q(\beta)$.

4. para $\alpha>0$ um ordinal limite, $p \in P_{\alpha}$ se, e somente se, para todo $\beta<\alpha, P_{\beta}=\left\{p \mid \beta: p \in P_{\alpha}\right\}$ é uma iteração de comprimento $\beta<\alpha$ e $\left\{\beta<\alpha: P_{\beta} \forall p(\beta)=\mathbf{1}_{\dot{Q}}\right\}$ é finito; a ordem em $P_{\alpha}$ é dada por $p \leq q$ se, e somente se, $p\left|\beta \leq_{P_{\beta}} q\right| \beta$, para todo $\beta<\alpha$.

Se $p \in P_{\alpha}$, definimos o suporte de $p$, que denotaremos por $\operatorname{supp}(p)$ o conjunto $\left\{\beta<\alpha: P_{\beta} \not \forall p(\beta)=1_{\dot{Q}}\right\}$. Se $G$ é um $P_{\alpha}$-genérico sobre $M$ e $\beta<\alpha$ definimos

$$
G_{\beta}=\{p \mid \beta: p \in G\}
$$

e

$$
G_{\{\beta\}}=\{p(\beta): p \in G\} .
$$

Teorema B.21. ([Ku], VIII, 5.13) Sejam $M$ um modelo transitivo enumerável e $P_{\alpha} \in M$ um forcing iterado de comprimento $\alpha$. Sejam $\beta<\alpha$ e $G$ é um $P_{\alpha}$-genérico sobre $M$. Então

(a) $G_{\beta}$ é um $P_{\beta}$-genérico sobre $M$;

(b) $M\left[G_{\beta}\right] \subseteq M[G]$.

Teorema B.22. ([Je], 16.9) Sejam $M$ um modelo transitivo enumerável e $P_{\alpha} \in M$ um forcing iterado de comprimento $\alpha$, e sejam $\left(\dot{Q}_{\beta}\right)_{\beta<\alpha}$ tais que $P_{\beta+1}=P_{\beta} * \dot{Q}_{\beta}$ e $P_{\beta} \Vdash$ " $\dot{Q}_{\beta}$ é c.c.c.", para todo $\beta<\alpha$. Entâo $P_{\alpha}$ é c.c.c. 
Forcing 


\section{Bibliografia}

[AG] Aiena, P.; Gonzalez, M. Essentially incomparable Banach spaces and Fredholm Theory. Proc. Irish. Acad. Sect. A93, n. 1, p. 49 - 59, 1993.

[Br] Brech, C. On the density of Banach spaces $C(K)$ with the Grothendieck property. Proc. Amer. Math. Soc., v. 134, p. 3653 - 3663, 2006.

[Ci] Ciesielski, K. Set theory for the working mathematician. Cambridge University Press, Cambridge, 1997.

[Di] Diestel, J. Sequences and series in Banach spaces. Spring-Verlag, New York, 1984.

[DU] Diestel, J.; Uhl, J. J. Jr. Vector measures. Mathematical Surveys 15, AMS. 1977.

[Do] Douwen, E. K. The integers and topology. Em Kunen, K.; Vaughan, J. E., editores, Handbook of set-theoretic topology. Elsevier Science Publishers B.V., p. 111 - 167, 1984.

[DS] Dunford, N.; Schwartz, J. T. Linear operators. Interscience Publishers LTD., London, 1958.

[Eng] Engelking, R. General topology, $2^{\mathrm{a}}$ ed. Sigma Series in Pure Mathematics, 6, Heldermann Verlag, Berlin, 1989.

[Fa] Fabian, M.; Hájek, P.; Pelant, J.; Habala, P.; Montesinos, V.; Zizler, V. Functional analysis and infinite-dimensional geometry. Spring-Verlag, New York, 2001

[Fed] Fedorchuk, V.V. A bicompactum whose infinite closed subsets are all n-dimensinal (Russo). Math. USSR Sbornik, v. 25, p. 37 - 57, 1975.

[Fer] Ferenczi, V. Quotient hereditarily indecomposable Banach spaces. Canad. J. Math., v. 51, n.3, p. 566 - 584, 1999.

[Fr] Fremlin, D. H. Consequences of Martin's axiom. Cambridge University Press, Cambridge, 1984. 
[Ga] Gasparis, I. A continuum of totally incomparable hereditarily indecomposable Banach spaces. Studia Math., v. 151, n. 3, p. 277 - 298, 2002.

[GM1] Gowers, W. T; Maurey, B. The unconditional basic sequence problem. Journal A. M. S., v. 6, p. 851-874, 1993.

[GM2] Gowers, W. T; Maurey, B. Banach spaces with small spaces of operators. Math. Ann., v. 307, p. 543-568, 1997.

[Ha] Haydon, R.A non-reflexive Grothendieck space that does not contain $l_{\infty}$. Israel J. Math., v. 40, n.1, p.65-73, 1981.

[HLO] Haydon, R.; Levy, M.; Odell, E. On sequences without weak* convergent convex block subsequences. Proc. Amer. Math. Soc., v.100, n.1, p. 94 98, 1987.

[Je] Jech, T. Set Theory. Second edition. Perspectives in Mathematical Logic. Springer-Verlag, Berlin, 1997.

[KP] Kadec, M. I.; Pełczyński, A. Bases, lacunary sequences and complemented subspaces in the space $L_{p}$. Studia Math., v. 21, p. 161 - 176, 1962.

[Ko1] Koszmider, P. Forcing minimal extensions of Boolean algebras. Trans. Amer. Math. Soc., v. 351, n.8, p. 3073 - 3117, 1999.

[Ko2] Koszmider, P. Banach spaces of continuous functions with few operators. Math. Annalen., v. 330, p. 151-183, 2004.

[Ko3] Koszmider, P. A space $C(K)$ where all nontrivial complemented subspaces have big densities. Studia Math., v. 168, n.2, p. 109 - 127, 2005.

[Kop] Koppelberg, S. General theory of Boolean algebras. Em Monk, J.D., editor, Handbook of Boolean Algebras. Elsevier Science Publishers B.V., Amsterdam, 1989.

$[\mathrm{Ku}]$ Kunen, K. Set Theory. An Introduction to Independence Proofs. North Holland, 1980.

[LM] Lacey, E.; Morris, P. On spaces of the type $A(K)$ and their duals. Proc. Amer. Math. Soc., v. 23, p. 151 - 157, 1969

[Mi] Mibu; On Baire functions on finite product spaces. Proc. Imperial Acad. Tokyo, v. 20, p. 661 - 663, 1994.

[Pe1] Pełczyński, A. Banach spaces on which every unconditionally converging operation is weakly compact. Bull. Acad. Polon. Sc. Ser. Sci. Math. Astronom. Phys., v.10, p. 641 - 648, 1962.

[Pe2] Pełczyński, A. On strictly singular and strictly non-singular operators I. Bull. Acad. Polon. Sc. Ser. Sci. Math. Astronom. Phys., v.13, p. 31 - 
41, 1962.

[Pl] Plebanek, G.; A construction of a Banach space $C(K)$ with few operators. Top. Appl., v. 143, p. 217 - 239, 2004.

[Ru] Rudin, W. Real and Complex Analysis, $3^{\mathrm{a}}$ ed. McGraw-Hill, New York, 1987.

[Sc] Schachemayer, W. On some classical measure-theoretic theorems for nonsigma-complete Boolean algebras. Diss. Math., 214, 1982.

[Schl] Schlackow, I. Centripetal operators and Koszmider spaces. (preprint), 2007

[Se] Semadeni, Z.Banach spaces of continuous functions. Państwowe Wydawnictwo Naukowe, 1971

[Sh] Shelah, S. A Banach space with few operators. Israel J. Math., v. 30, p. 181 - 191, 1978.

[SS] Shelah, S.; Steprans, J.; A Banach Space on which there are few operators. Proc. Amer. Math. Soc., v. 104, p. 101 - 105, 1988.

[Ta] Talagrand, M. Un Nouveau $C(K)$ qui possede la propriete de Grothendieck. Israel J. Math., v. 37, p. 181 - 191, 1980.

[Ve] Velleman, D. Morasses, diamond and forcing. Ann. Pure. Appl. Logic, v. 23, p. 199-281, 1983. 


\title{
Índice Remissivo
}

\author{
$C(K), 1$ \\ $M(K), 9$ \\ $M[G], 95$ \\ $M^{P}, 95$ \\ $P$-nome, 95 \\ $V^{P}, 95$ \\ $\Delta_{0}, 98$ \\ $\Gamma, 95$ \\ $\Vdash, 96$ \\ $\beta \mathbb{N}, 3,90$ \\ $\check{x}, 95$ \\ $\diamond, 1,20,27$ \\ $\mathcal{L}\left(M^{P}\right), 96$ \\ $\sigma$-álgebra, 7 \\ $\sigma$-aditiva, 7 \\ $\tau_{G}, 95$ \\ condições do forcing, 93 \\ conjunto denso (em um forcing), 93 \\ consistente, 91, 93 \\ decomponível, 1 \\ densidade, 4 \\ espaço com poucos operadores, 1, 2 \\ espaço de Stone, 9 \\ espaços booleanos, 87 \\ essencialmente incomparáveis, 4, 44 \\ estacionário, 20, 27 \\ estritamente singular, 1, 2 \\ extensão, 13 \\ extensão forte, 13 \\ $\varepsilon$-fracamente relativamente compacto, \\ 50 \\ álgebra de Boole, 9 \\ fórmula absoluta, 97 \\ fórmula atômica, 91 \\ fechado ilimitado, 20, 27, 57, 77 \\ filtro genérico, 94 \\ 0-dimensional, 12, 41 \\ forcing, 5, 91, 93 \\ forcing c.c.c., 99, 101 \\ aberto elementar, 9 \\ forcing iterado, 5, 99 \\ anticadeia, 94 \\ axioma de Martin, 4 \\ forcing trivial, 93 \\ fracamente compacto, 2, 10 \\ borelianos, 7 \\ função boreliana, 9 \\ classe, 92,95 \\ compactificado de Stone-Čech, 90 \\ hereditariamente indecomponível, 1 \\ Hipótese do Contínuo, 2 \\ compatíveis, 93 \\ incompatíveis, 93 \\ complementado, 1 \\ indecomponível, 1
}


independente, 49, 91

Lema de Rosenthal, 10

limite em sistema dirigido, 71

limite inverso, 14

medida absolutamente contínua, 53

medida boreliana, 7

medida de Radon, 7

medida positiva, 7

medida regular, 7

medidas duas a duas disjuntas, 51

modelo standard, 92

modelo transitivo, 92

multiplicador fraco, 11

operador de Fredholm, 43

ordem parcial, 93

preservação de cardinais, 99

Princípio do forcing, 93

propriedade de Grothendieck, 4

quociente hereditariamente indecomponível, 2

raro, 13

relação de forcing, 97

relativamente consistente, 91, 93

relativização, 92

sentença, 92

soma direta, 1

$\operatorname{supp}(\mathrm{p}), 101$

Teorema de Alaoglu, 64

Teorema de Dieudonné-Grothendieck, 9

Teorema de Eberlein-Šmulian, 10

Teorema de Gantmacher, 10
Teorema de Radon-Nikodym, 53

totalmente desconexo, 41

totalmente incomparáveis, 4

variável livre, 92

variação, 7

ZFC, 49, 92 